\title{
Non-local Markovian Symmetric Forms on Infinite Dimensional Spaces I. The closability and quasi-regularity
}

\author{
Sergio Albeverio ${ }^{1}$, Toshinao Kagawa ${ }^{2}$, Yumi Yahagi ${ }^{3}$, Minoru W. Yoshida ${ }^{2}$ (D \\ ${ }^{1}$ Inst. Angewandte Mathematik, and HCM, University of Bonn, Bonn, Germany \\ E-mail: albeverio@iam.uni-bonn.de \\ 2 Department Information Systems, Kanagawa University, Yokohama, Japan \\ E-mail: washizuminoru@ @otmail.com \\ 3 Department Mathematical information Tokyo University of Information, Chiba, Japan
}

Received: 7 October 2019 / Accepted: 25 July 2021

(C) The Author(s) 2021

\begin{abstract}
General theorems on the closability and quasi-regularity of non-local Markovian symmetric forms on probability spaces $(S, \mathcal{B}(S), \mu)$, with $S$ Fréchet spaces such that $S \subset \mathbb{R}^{\mathbb{N}}, \mathcal{B}(S)$ is the Borel $\sigma$-field of $S$, and $\mu$ is a Borel probability measure on $S$, are introduced. Firstly, a family of non-local Markovian symmetric forms $\mathcal{E}_{(\alpha)}$, $0<\alpha<2$, acting in each given $L^{2}(S ; \mu)$ is defined, the index $\alpha$ characterizing the order of the non-locality. Then, it is shown that all the forms $\mathcal{E}_{(\alpha)}$ defined on $\bigcup_{n \in \mathbb{N}} C_{0}^{\infty}\left(\mathbb{R}^{n}\right)$ are closable in $L^{2}(S ; \mu)$. Moreover, sufficient conditions under which the closure of the closable forms, that are Dirichlet forms, become strictly quasi-regular, are given. Finally, an existence theorem for Hunt processes properly associated to the Dirichlet forms is given. The application of the above theorems to the problem of stochastic quantizations of Euclidean $\Phi_{d}^{4}$ fields, for $d=2,3$, by means of these Hunt processes is indicated.
\end{abstract}

\section{Introduction}

We consider a space $S$ that is either a real Banach space $l^{p}, 1 \leq p \leq \infty$, with suitable weights, or the direct product space $\mathbb{R}^{\mathbb{N}}$ (with $\mathbb{R}$ and $\mathbb{N}$ the spaces of real numbers and natural numbers, respectively). Both will be looked upon as Fréchet spaces. Let $\mu$ be a Borel probability measure on $S$. On the real $L^{2}(S ; \mu)$ space, for each $0<\alpha<2$, we give an explicit formulation of $\alpha$-stable type non-local quasi-regular (cf. section IV-3 of [69]) Dirichlet forms $\left(\mathcal{E}_{(\alpha)}, \mathcal{D}\left(\mathcal{E}_{(\alpha)}\right)\right.$ ) (with a domain $\mathcal{D}\left(\mathcal{E}_{(\alpha)}\right)$ ), and show the existence of $S$-valued Hunt processes properly associated to $\left(\mathcal{E}_{(\alpha)}, \mathcal{D}\left(\mathcal{E}_{(\alpha)}\right)\right)$. $\alpha$-stable is understood in analogy with the $\alpha$-stable Dirichlet forms defined on $L^{2}\left(\mathbb{R}^{d}\right)$, for $d \in \mathbb{N}$, e.g., in [49], Sect. 5.

As an application of the above general results, in Example 1 and 2 in Sect. 5, we consider the problem of stochastic quantization of the Euclidean free field over $\mathbb{R}^{d}$, the $\Phi_{2}^{4}$ and $\Phi_{3}^{4}$ fields over $\mathbb{R}^{2}$ and $\mathbb{R}^{3}$, i.e., fields with no (self) interaction respectively, (self) interaction of the 4-th power. By using the property that, for example, the support of the Euclidean $\Phi_{3}^{4}$ field measure $\mu$ is in some real Hilbert space $\mathcal{H}_{-3}$ (cf. (5.9) for 
the explicit definition), which is a subspace of the Schwartz space of real tempered distributions $\mathcal{S}^{\prime}\left(\mathbb{R}^{3} \rightarrow \mathbb{R}\right)$, we define an isometric isomorphism $\tau_{-3}$ from $\mathcal{H}_{-3}$ to "some weighted $l^{2}$ space" (cf. (5.22) for the explicit definition). By making use of $\tau_{-3}$, we then apply the above general theorems formulated on the abstract $L^{2}(S ; \mu)$ space to the case of the space $L^{2}\left(\mathcal{H}_{-3} ; \mu\right)$ for the Euclidean $\Phi_{3}^{4}$ field, and for each $0<\alpha \leq 1$ we show the existence of an $\mathcal{H}_{-3}$-valued Hunt process $\left(Y_{t}\right)_{t \geq 0}$ the invariant measure of which is $\mu$.

$\left(Y_{t}\right)_{t \geq 0}$ can be understood as a stochastic quantization of the Euclidean $\Phi_{3}^{4}$ field realized by a Hunt process through the non-local Dirichlet form $\left(\mathcal{E}_{(\alpha)}, \mathcal{D}\left(\mathcal{E}_{(\alpha)}\right)\right.$ ) for $0<\alpha \leq 1$, in the sense that $\Phi_{3}^{4}$-measure $\mu$ is the invariant measure for $\left(Y_{t}\right)_{t \geq 0}$.

(1) As far as we know, there has been no explicit proposal of a general formulation of non-local quasi-regular Dirichlet forms on infinite dimensional (Fréchet) topological vector spaces, which admit interpretations as Dirichlet forms on concrete random fields on Fréchet spaces. This is different from the situation associated with the local case, i.e., the case where the associated Markov processes are (continuous) diffusions, where much has been developed and known (cf. a short review given below).

(2) Although there have been derived several results on the existence of (continuous) diffusions (i.e., roughly speaking, that are associated to quadratic forms and generators of local type) corresponding with stochastic quantizations of, e.g., $\Phi_{2}^{4}$ and $\Phi_{3}^{4}$ Euclidean fields (see below for references), as far as we know, there do not exist no explicit corresponding considerations for non-local type Markov processes, associated with non-local Dirichlet forms related to such fields. The only examples so far provided have been obtained by subordination, for the Euclidean free field (on $\mathcal{S}^{\prime}\left(\mathbb{R}^{d}\right), d \geq 1$ ) and for the $\Phi_{2}^{4}$-Euclidean field with space cut-off (interaction in a bounded euclidean space region) a non-local type stochastic quantization procedure has been discussed in [32](see (5.29), (5.62) and Remark 12).

The present paper is a first development that gives answers to the above mentioned open problems 1), i.e., to give an abstract formulation in this concern, and 2), i.e., to perform a corresponding consideration in the case of $\Phi_{3}^{4}$-model.

Before giving an explanation of the contents of the present paper, we give a brief review of the theory of Dirichlet forms and its applications to stochastic quantization having correspondences with the present considerations.

In the case where the state space $E$ is finite dimensional, much has been done on the considerations of both local and non-local Dirichlet forms and semi-Dirichlet forms (non-symmetric Dirichlet forms). The natural setting is the one where the Hilbert space, where the Dirichlet forms are defined (as quadratic forms), is $L^{2}(E ; m)$, with $E$ a general locally compact separable metric space (when $E$ is a topological vector space, the dimension of the space is thus finite) and $m$ a positive Radon measure on it (cf., e.g., [46,48,49,60,70,84,88], [4], [34,35] and references therein). Also, many results have been developed on the theory of general (non-symmetric) local Dirichlet forms defined on $L^{2}(E ; m)$, with general topological spaces including the case of some infinite dimensional topological vector spaces, and $m$ some Radon measures on them (cf., e.g., [3,14-17,25-27,29-32,68,69,85,87] and references therein). In the general abstract framework, in particular [3,69], the Dirichlet forms need not be local ones, however all examples except those considered through the framework of subordination given in [A,Rüdiger] (cf. Remark 12 in Sect. 5) treated in above references, treating the case where $E$ is infinite dimensional, concern Dirichlet forms theories that are local ones (i.e., associated with differential operators). 
One of the most important applications of the theory of Dirichlet forms on $L^{2}(E ; m)$, $E$ an infinite dimensional space, is to construct an $E$-valued Markov process, the invariant measure of which is $m$ (assuming $m$ to be a probability measure). In particular, if $L^{2}(E ; m)$ is a space associated to the Euclidean quantum field (for the construction of $m$ cf., e.g., [52,89]), the constructed Markov process is referred to as stochastic quantization of the field. In the literature quoted above concerning local Dirichlet forms we find several applications to stochastic quantizations, where the Markov processes constructed are (continuous) diffusion processes associated to local Dirichlet forms. For the stochastic quantizations of Euclidean $P(\phi)_{2}$ fields on $\mathcal{S}^{\prime}\left(\mathbb{R}^{2} \rightarrow \mathbb{R}\right)$, i.e., fields with polynomial interactions, fields with trigonometric interactions and exponential interactions on $\mathcal{S}^{\prime}\left(\mathbb{R}^{2} \rightarrow \mathbb{R}\right)$, these considerations by means of the local Dirichlet form arguments were completed by [12-17,25-27,29-31,37,59]. In this direction of the application of local Dirichlet forms, there also are corresponding considerations for measures $m$ describing infinite particle systems (cf., e.g., [21,35,75,92] and references therein). For works on stochastic quantization of $\Phi_{2}^{4}$ using other methods see [40,41,65] and references in [Albeverio, Ma, Röckner], [A, Kusuoka-sei 2017]. For other models in two or less Euclidean space dimensions where stochastic quantization methods have been applied see the recent work [5,6] and references therein.

The problem of stochastic quantization of the $\Phi_{3}^{4}$ Euclidean field on the 3-dimensional torus $\mathbb{T}^{3}$ was solved firstly by [56], by not passing through the arguments by means of the Dirichlet form theory, but through the stochastic partial differential equation (SPDE in short) arguments with the theory of regularity structures developed there. A little more precisely, in [56] and subsequent works (see below) the existence of a solution, that is a (continuous) diffusion, of an SPDE, which were expected to be satisfied by a solution of the stochastic quantization of an Euclidean $\Phi_{3}^{4}$ model, restricted to $\mathbb{T}^{3}$, is shown (cf., e.g., Theorem 1.1 of [24]). Since the appearance of [56], there have been developed several results on (continuous) diffusion type stochastic quantization of the $\Phi_{3}^{4}$ Euclidean field (cf., e.g., [39,57,73] [22,23,54,55] and references therein). [Zu,Zu1 2018], [Zu,Zu2 2018] considered the existence of a local Dirichlet form corresponding to the Euclidean $\Phi_{3}^{4}$ field on $\mathbb{T}^{3}$ by making use of the result on existence of the solution of the SPDE by [56].

Now, let us give a brief summary of the contents of the single sections of this work. Theorems 1-4 are statements corresponding to the non-local Dirichlet forms constructed explicitly on $L^{2}(S ; \mu)$, where $S$ denotes the weighted $l^{p}, 1 \leq p \leq \infty$, spaces and subspace of the direct product $\mathbb{R}^{\mathbb{N}}$, respectively, and $\mu$ is a Borel probability measure on $S$. Theorem 5 is a restatement of the Bochner-Minlos theorem in this framework. Theorem 6 contains an application to a non-local type stochastic quantization of the Euclidean $\Phi_{d}^{4}, d=2,3$, theory, derived by applying the above general theorems to the $\Phi_{d}^{4}$ field by making use of an isometric isomorphism between a Hilbert space, taken as support of the $\Phi_{d}^{4}$ field measure, and a weighted $l^{2}$ space on which Theorems 1,2 and 4 are considered.

In Sect. 2, for each $0<\alpha<2$ we define the symmetric Markovian form $\mathcal{E}_{(\alpha)}$ on $L^{2}(S ; \mu)($ cf. (2.8), (2.9) and (2.10)), which is given by using the conditional probability of $\mu$, where the index $\alpha$ characterizes the order of the non-locality. The definition is a natural analogue of the one for $\alpha$-stable type non local Dirichlet forms on $\mathbb{R}^{d}, d<\infty$ (cf. (2.13) in Remark 2 of the present paper and (5.3), (1.4) of [49]). It has a natural correspondence to the one for local classical Dirichlet forms on infinite dimensional topological vector spaces (cf. [29-31] and [68]), which is carried out by making use of directional derivatives. Theorem 1 shows that the non-local symmetric forms $\mathcal{E}_{(\alpha)}$ with 
the domain $\mathcal{F} C_{0}^{\infty}$, the space of regular cylindrical functions (cf. (2.7)), are closable in $L^{2}(S ; \mu)$, hence their closures are non-local Dirichlet forms denoted by $\left(\mathcal{E}_{(\alpha)}, \mathcal{D}\left(\mathcal{E}_{(\alpha)}\right)\right)$.

We give a proof of Theorem 1 in Sect. 3, by considering separately the cases $0<$ $\alpha \leq 1$ and $1<\alpha<2$.

Section 4 discusses strictly quasi-regularity (cf., e.g., section IV-3 and section V-2 of [M,,R 92]) of the non-local Dirichlet form $\left(\mathcal{E}_{(\alpha)}, \mathcal{D}\left(\mathcal{E}_{(\alpha)}\right)\right)$ on $L^{2}(S ; \mu)$, by which the existence of Hunt processes properly associated with $\left(\mathcal{E}_{(\alpha)}, \mathcal{D}\left(\mathcal{E}_{(\alpha)}\right)\right)$ is guaranteed. Theorems 2 and 3 give sufficient conditions under which $\left(\mathcal{E}_{(\alpha)}, \mathcal{D}\left(\mathcal{E}_{(\alpha)}\right)\right)$ for $0<\alpha \leq 1$ and for $1<\alpha<2$ become strictly quasi-regular, respectively. In the case where $S$ is a weighted $l^{p}, 1 \leq p \leq \infty$, space, the proofs are carried out by making an efficient use of the structures of weighted spaces. In short, the concept of the quasi-regularity on $\mathcal{D}\left(\mathcal{E}_{(\alpha)}\right)$ is a requirement that $\mathcal{D}\left(\mathcal{E}_{(\alpha)}\right)$ contains a sequence of subsets which is dense in $\mathcal{D}\left(\mathcal{E}_{(\alpha)}\right)$, the elements of which are functions on $S$ with compact supports. By making use of the weight of the $l^{p}$ spaces, compact sets in $S$ can be given explicitly (cf. (4.15)), and then a corresponding sequence of subsets in $\mathcal{D}\left(\mathcal{E}_{(\alpha)}\right)$ can be constructed.

Theorem 4 in Sect. 5 gives the statement of the existence of $S$-valued Hunt processes that are properly associated to the non-local strictly quasi-regular Dirichlet forms $\left(\mathcal{E}_{(\alpha)}, \mathcal{D}\left(\mathcal{E}_{(\alpha)}\right)\right)$ on $L^{2}(S ; \mu)$ given by Theorems 2 and 3 . In the same section, we recall the Bochner-Minlos theorem (cf. Theorem 5), and then by making use of this theorem and Theorem 4 we solve the problems of stochastic quantization of the Euclidean free field on $\mathcal{S}^{\prime}\left(\mathbb{R}^{d} \rightarrow \mathbb{R}\right), d \in \mathbb{N}$, and the Euclidean $\Phi_{d}^{4}$ field on $\mathcal{S}^{\prime}\left(\mathbb{R}^{d} \rightarrow \mathbb{R}\right), d=2,3$ in Example 1, and in Example 2, respectively. As a consequence, the stochastic quantizations are realized by Hunt processes properly associated to the non-local Dirichlet forms $\left(\mathcal{E}_{(\alpha)}, \mathcal{D}\left(\mathcal{E}_{(\alpha)}\right)\right)$ for $0<\alpha \leq 1$. In these examples, in order to apply Theorem 4 to the Euclidean free field measure and to the $\Phi_{2}^{4}$ and $\Phi_{3}^{4}$ field measures, respectively, on the Schwartz space of tempered distributions, we firstly certify that these measures have support in the Hilbert spaces $\mathcal{H}_{-2}$ and $\mathcal{H}_{-3}$ (cf. (5.11)), respectively, and define the isometric isomorphism

$$
\tau_{-2}: \mathcal{H}_{-2} \rightarrow \text { a weighted } l^{2} \text { space }, l_{\left(\lambda_{i}^{4}\right)}^{2}(c f .(5.27))
$$

and,

$$
\tau_{-3}: \mathcal{H}_{-3} \rightarrow \text { a weighted } l^{2} \text { space, } l_{\left(\lambda_{i}^{6}\right)}^{2}(c f .(5.56))
$$

and we then identify $\mathcal{H}_{-2}$ with $l_{\left(\lambda_{i}^{4}\right)}^{2}$ and $\mathcal{H}_{-3}$ with $l_{\left(\lambda_{i}^{6}\right)}^{2}$, respectively. For the weighted $l^{2}$ spaces $l_{\left(\lambda_{i}^{4}\right)}^{2}$ and $l_{\left(\lambda_{i}^{6}\right)}^{2}$ we can apply Theorems 1, 2, 4 and have $l_{\left(\lambda_{i}^{4}\right)}^{2}$ and $l_{\left(\lambda_{i}^{6}\right)}^{2}$ valued Hunt processes $\left(X_{t}\right)_{t \geq 0}$ properly associated to corresponding Dirichlet forms $\left(\mathcal{E}_{(\alpha)}, \mathcal{D}\left(\mathcal{E}_{(\alpha)}\right)\right)$, respectively (in case of $\alpha=1$, for the Euclidean free field on $\mathcal{S}^{\prime}\left(\mathbb{R}^{d} \rightarrow \mathbb{R}\right), d \in \mathbb{N}$ an $l_{\left(\lambda_{i}^{4}\right)}^{2}$-valued Hunt process is defined, and generally, for $0<\alpha \leq 1, l_{\left(\lambda_{i}^{6}\right)}^{2}$-valued Hunt processes are defined for both the Euclidean free field on $\mathcal{S}^{\prime}\left(\mathbb{R}^{d} \rightarrow \mathbb{R}\right), d \in \mathbb{N}$ and the Euclidean $\Phi_{d}^{4}$ field on $\left.\mathcal{S}^{\prime}\left(\mathbb{R}^{d} \rightarrow \mathbb{R}\right), d=2,3\right)$. Finally, we define the corresponding $\mathcal{H}_{-2}$ and $\mathcal{H}_{-3}$ valued Hunt processes $\left(Y_{t}\right)_{t \geq 0}$ which are inverse images of $\left(X_{t}\right)_{t \geq 0}$ through $\tau_{-2}$ and $\tau_{-3}$, respectively (see Theorem 6).

In Sect. 6, we give a short outlook to future developments, in the line of the present formulations and results. 


\section{Markovian Symmetric Forms Individually Adapted to Each Measure Space}

The state space $S$, on which we define the Markovian symmetric forms, is one of the following Fréchet spaces (i.e., complete infinite dimensional topological vector spaces with a system of countable semi-norms): A weighted $l^{p}$ space, denoted by $l_{\left(\beta_{i}\right)}^{p}$, such that, for some $p \in[1, \infty)$ and a weight $\left(\beta_{i}\right)_{i \in \mathbb{N}}$ with $\beta_{i} \geq 0, i \in \mathbb{N}$,

$$
S=l_{\left(\beta_{i}\right)}^{p} \equiv\left\{\mathbf{x}=\left(x_{1}, x_{2}, \ldots\right) \in \mathbb{R}^{\mathbb{N}}:\|\mathbf{x}\|_{l_{\left(\beta_{i}\right)}^{p}} \equiv\left(\sum_{i=1}^{\infty} \beta_{i}\left|x_{i}\right|^{p}\right)^{\frac{1}{p}}<\infty\right\},
$$

or a weighted $l^{\infty}$ space, denoted by $l_{\left(\beta_{i}\right)}^{\infty}$, such that for a weight $\left(\beta_{i}\right)_{i \in \mathbb{N}}$ with $\beta_{i} \geq 0, i \in$ $\mathbb{N}$,

$$
S=l_{\left(\beta_{i}\right)}^{\infty} \equiv\left\{\mathbf{x}=\left(x_{1}, x_{2}, \ldots\right) \in \mathbb{R}^{\mathbb{N}}:\|\mathbf{x}\|_{l_{\left(\beta_{i}\right)}^{\infty}} \equiv \sup _{i \in \mathbb{N}} \beta_{i}\left|x_{i}\right|<\infty\right\}
$$

or

$$
S=\mathbb{R}^{\mathbb{N}} \text {, the direct product space with the metric } d(\cdot, \cdot)
$$

such that for $\mathbf{x}, \mathbf{x}^{\prime} \in \mathbb{R}^{\mathbb{N}}$,

$$
\begin{aligned}
& d\left(\mathbf{x}, \mathbf{x}^{\prime}\right) \equiv \sum_{k=1}^{\infty}\left(\frac{1}{2}\right)^{k} \frac{\left\|\mathbf{x}-\mathbf{x}^{\prime}\right\|_{k}}{\left\|\mathbf{x}-\mathbf{x}^{\prime}\right\|_{k}+1}, \\
& \text { with }\|\mathbf{x}\|_{k}=\left(\sum_{i=1}^{k}\left(x_{i}\right)^{2}\right)^{\frac{1}{2}}, \mathbf{x}=\left(x_{1}, x_{2}, \ldots\right) \in \mathbb{R}^{\mathbb{N}} .
\end{aligned}
$$

By making use of the concrete expressions of $S$ as above, i.e., their expressions by means of a subspaces of $\mathbb{R}^{\mathbb{N}}$, abstract discussions on Dirichlet forms (i.e., closed Markovian symmetric forms, on $L^{2}(S ; \mu)$ ) can be made more concrete. Moreover, our choice of $S$ permits an effective application of the theory of Dirichlet forms to certain problems related to the stochastic quantization of Euclidean quantum fields (cf. Remark in the last section).

We denote by $\mathcal{B}(S)$ the Borel $\sigma$-field of $S$. Suppose that we are given a Borel probability measure $\mu$ on $(S, \mathcal{B}(S))$. For each $i \in \mathbb{N}$, let $\sigma_{i^{c}}$ be the sub $\sigma$-field of $\mathcal{B}(S)$ that is generated by the Borel sets

$$
\begin{aligned}
& B \equiv\left\{\mathbf{x} \in S \mid x_{j_{1}} \in B_{1}, \ldots x_{j_{n}} \in B_{n}\right\}, \\
& j_{k} \neq i, \quad B_{k} \in \mathcal{B}^{1}, k=1, \ldots, n, n \in \mathbb{N},
\end{aligned}
$$

where $\mathcal{B}^{1}$ denotes the Borel $\sigma$-field of $\mathbb{R}^{1}$, i.e., $\sigma_{i}$ is the smallest $\sigma$-field that includes every $B$ given by (2.4). Namely, $\sigma_{i}{ }^{c}$ is the sub $\sigma$-field of $\mathcal{B}(S)$ generated by the variables $\mathbf{x} \backslash x_{i}$, i.e., the variables except of the $i$-th variable $x_{i}$. For each $i \in \mathbb{N}$, let $\mu\left(\cdot \mid \sigma_{i^{c}}\right)$ be the conditional probability, a one-dimensional probability distribution-valued $\sigma_{i^{c}}$ measurable function (i.e., the probability distribution for the $i$-th component $x_{i}$ ), that is characterized by (cf. (2.4) of [31])

$$
\mu\left(\left\{\mathbf{x}: x_{i} \in A\right\} \cap B\right)=\int_{B} \mu\left(A \mid \sigma_{i^{c}}\right) \mu(d \mathbf{x}), \quad \forall A \in \mathcal{B}^{1}, \forall B \in \sigma_{i^{c}} .
$$


Define

$$
L^{2}(S ; \mu) \equiv\left\{f \mid f: S \rightarrow \mathbb{R}, \text { measurable and }\|f\|_{L^{2}}=\left(\int_{S}|f(\mathbf{x})|^{2} \mu(d \mathbf{x})\right)^{\frac{1}{2}}<\infty\right\},
$$

and

$$
\mathcal{F} C_{0}^{\infty} \equiv \text { the } \mu \text { equivalence class of }\left\{f \mid \exists n \in \mathbb{N}, f \in C_{0}^{\infty}\left(\mathbb{R}^{n} \rightarrow \mathbb{R}\right)\right\} \subset L^{2}(S ; \mu),
$$

where $C_{0}^{\infty}\left(\mathbb{R}^{n} \rightarrow \mathbb{R}\right)$ denotes the space of real valued infinitely differentiable functions on $\mathbb{R}^{n}$ with compact supports (cf. (4.29) below).

Remark 1. (i) For the subsequent discussions, in particular those concerning the closability of quadratic forms on $L^{2}(S ; \mu)$, we first have to certify that $\mathcal{F} C_{0}^{\infty}$ defined by (2.7) is dense in $L^{2}(S ; \mu)$. This can be seen through the same argument performed in section II-3-a) of [69] for a Souslin space, where the corresponding discussion is carried out for $\mathcal{F} C_{b}^{\infty}$ that is defined by substituting $C_{0}^{\infty}\left(\mathbb{R}^{n} \rightarrow \mathbb{R}\right)$ in (2.7) by $C_{b}^{\infty}\left(\mathbb{R}^{n} \rightarrow \mathbb{R}\right)$ (see (3.1) in section II-3 of [69]). But in the present formulation where the state space $S$ is given by (2.1), (2.2) or (2.3), the fact that $\mathcal{F} C_{0}^{\infty}$ is dense in $L^{2}(S ; \mu)$ can be seen directly as follows: The Borel $\sigma$-field $\mathcal{B}(S)$ of $S$ is generated by the collection of finite direct products of the sets such that $\left\{x_{i}: a_{i} \leq x_{i} \leq b_{i}\right\}$, $(i \in \mathbb{N})$, and for each indicator function $I_{\left[a_{i}, b_{i}\right]}\left(x_{i}\right)$ there exists a sequence of functions $\left\{f_{n}\left(x_{i}\right)\right\}_{n \in \mathbb{N}}, f_{n}(\cdot) \in C_{0}^{\infty}(\mathbb{R}), n \in \mathbb{N}$, that has a uniform bound, such that it converges to $I_{\left[a_{i}, b_{i}\right]}\left(x_{i}\right)$ in $L^{2}(S ; \mu)$ by the Lebesgue's bounded convergence theorem.

(ii) Let $C(S \rightarrow \mathbb{R})$ be the space of real valued continuous functions on $S$. For each $S$ that is given by (2.1), (2.2) or (2.3), the fact that $\mathcal{F} C_{0}^{\infty} \subset C(S \rightarrow \mathbb{R})$ can be also seen directly. But, since $S=\mathbb{R}^{\mathbb{N}}$ defined by (2.3) is equipped the weakest topology, and clearly $\mathcal{F} C_{0}^{\infty} \subset C\left(\mathbb{R}^{\mathbb{N}} \rightarrow \mathbb{R}\right)$, the same is also true for $S$ as given by (2.1) and (2.2).

(iii) The completeness of the metric space $S$ is not necessary for the discussions in this section, but it shall be used for the considerations of the quasi regularity of symmetric forms performed in Sect. 4.

On $L^{2}(S ; \mu)$, for any $0<\alpha<2$, let us define the Markovian symmetric form $\mathcal{E}_{(\alpha)}$ called individually adapted Markovian symmetric form of index $\alpha$ to the measure $\mu$, the definition of which is a natural analogue of the one for $\alpha$-stable type (non local) Dirichlet form on $\mathbb{R}^{d}, d<\infty$ (cf. Remark 2 given below and (5.3), (1.4) of [49]). The concept extends to the non local case the one of local classical Dirichlet forms on infinite dimensional topological vector spaces (cf. [29-31] and [68]), which is carried out by making use of directional derivatives. Our definition is as follows: Firstly, for each $0<$ $\alpha<2$ and $i \in \mathbb{N}$, and for the variables $y_{i}, y_{i}^{\prime} \in \mathbb{R}^{1}, \mathbf{x}=\left(x_{1}, \ldots, x_{i-1}, x_{i}, x_{i+1}, \ldots\right) \in S$ and $\mathbf{x} \backslash x_{i} \equiv\left(x_{1}, \ldots, x_{i-1}, x_{i+1}, \ldots\right)$, let

$$
\begin{aligned}
& \Phi_{\alpha}\left(u, v ; y_{i}, y_{i}^{\prime}, \mathbf{x} \backslash x_{i}\right) \\
& \equiv \frac{1}{\left|y_{i}-y_{i}^{\prime}\right|^{\alpha+1}} \times\left\{u\left(x_{1}, \ldots, x_{i-1}, y_{i}, x_{i+1}, \ldots\right)-u\left(x_{1}, \ldots, x_{i-1}, y_{i}^{\prime}, x_{i+1}, \ldots\right)\right\} \\
& \quad \times\left\{v\left(x_{1}, \ldots, x_{i-1}, y_{i}, x_{i+1}, \ldots\right)-v\left(x_{1}, \ldots, x_{i-1}, y_{i}^{\prime}, x_{i+1}, \ldots\right)\right\} .
\end{aligned}
$$


For each $0<\alpha \leq 1$ and $i \in \mathbb{N}$, define

$$
\mathcal{E}_{(\alpha)}^{(i)}(u, v) \equiv \int_{S}\left\{\int_{\mathbb{R}} I_{\left\{y: y \neq x_{i}\right\}}\left(y_{i}\right) \Phi_{\alpha}\left(u, v ; y_{i}, x_{i}, \mathbf{x} \backslash x_{i}\right) \mu\left(d y_{i} \mid \sigma_{i^{c}}\right)\right\} \mu(d \mathbf{x}),
$$

for any $u, v$ such that the right hand side of (2.9) is finite, where for a set $A$ and a variable $y, I_{A}(y)$ denotes the indicator function, and in the sequel, to simplify the notations, we denote $I_{\left\{y: y \neq x_{i}\right\}}\left(y_{i}\right)$ by, e.g., $I_{\left\{y_{i} \neq x_{i}\right\}}\left(y_{i}\right)$ or $I_{\left\{y_{i} \neq x_{i}\right\}}$.

By $\mathcal{D}_{i}$, we denote the subset of the space of real valued $\mathcal{B}(S)$-measurable functions such that the right hand side of (2.9) is finite for any $u, v \in \mathcal{D}_{i}$. Let us call $\left(\mathcal{E}_{(\alpha)}^{(i)}, \mathcal{D}_{i}\right)$ this form, $\mathcal{D}_{i}$ being its domain, and then define

$$
\mathcal{E}_{(\alpha)}(u, v) \equiv \sum_{i \in \mathbb{N}} \mathcal{E}_{(\alpha)}^{(i)}(u, v), \quad \forall u, v \in \bigcap_{i \in \mathbb{N}} \mathcal{D}_{i},
$$

For $y_{i} \neq y_{i}^{\prime},(2.8)$ is well defined for any real valued $\mathcal{B}(S)$-measurable functions $u$ and $v$. Moreover, for the Lipschiz continuous functions $\tilde{u} \in C_{0}^{\infty}\left(\mathbb{R}^{n} \rightarrow \mathbb{R}\right) \subset \mathcal{F} C_{0}^{\infty}$ and $\tilde{v} \in$ $C_{0}^{\infty}\left(\mathbb{R}^{m} \rightarrow \mathbb{R}\right) \subset \mathcal{F} C_{0}^{\infty}, n, m \in \mathbb{N}$ with compact supports, which are representatives of $u \in \mathcal{F} C_{0}^{\infty}$ and $v \in \mathcal{F} C_{0}^{\infty}$, respectively (see (2.7)), (2.9) and (2.10) are well defined (the right hand side of (2.10) has only a finite number of sums). In Theorem 1 given below we will see that (2.9) and (2.10) are well defined for $\mathcal{F} C_{0}^{\infty}$, the space of $\mu$-equivalent class, and in particular $\mathcal{F} C_{0}^{\infty} \subset\left(\cap_{i \in \mathbb{N}} \mathcal{D}_{i}\right)$.

For $1<\alpha<2$, we suppose that for each $i \in \mathbb{N}$, the conditional distribution $\mu\left(\cdot \mid \sigma_{i^{c}}\right)$ can be expressed by a locally bounded probability density function $\rho\left(\cdot \mid \sigma_{i^{c}}\right)$, $\mu$-a.e., i.e., $\mu\left(\cdot \mid \sigma_{i^{c}}\right)=\rho\left(\cdot \mid \sigma_{i^{c}}\right) d x$. Precisely (cf. (2.5) of [31]), there exists a $\sigma_{i^{c}}$-measurable function $0 \leq \rho\left(\cdot \mid \sigma_{i c}\right)$ on $\mathbb{R}^{1}$ and

$$
\mu\left(d y \mid \sigma_{i^{c}}\right)=\rho\left(y \mid \sigma_{i^{c}}\right) d y, \quad \mu-\text { a.e., }
$$

holds, with $\rho\left(\cdot \mid \sigma_{i^{c}}\right)$ a function such that for each $i \in \mathbb{N}$ and for any compact $K \subset \mathbb{R}$ there exists a bound $L_{K, i}<\infty$, which may depend on $i$ such that

$$
\text { ess } \sup _{y \in K} \rho\left(y \mid \sigma_{i^{c}}\right) \leq L_{K, i}, \quad \mu-\text { a.e. }
$$

where ess $\sup _{y \in K}$ is taken with respect to the Lebesgue measure on $\mathbb{R}^{1}$. Then define the non-local form $\mathcal{E}_{(\alpha)}(u, v)$, for $1<\alpha<2$, by the same formulas as (2.9) and (2.10), for all $u, v \in \cap_{i \in \mathbb{N}} \mathcal{D}_{i}$, where $\mathcal{D}_{i}$ denotes the subset of the space of real valued $\mathcal{B}(S)$ measurable functions such that the right hand side of (2.9), with a given $\alpha$ such that $1<\alpha<2$, is finite for any $u, v \in \mathcal{D}_{i}$.

Remark 2. (i) For the $\mathcal{B}(S)$ measurable function $\int_{\mathbb{R}} I_{\left\{y_{i} \neq x_{i}\right\}} \Phi_{\alpha}\left(u, v ; y_{i}, x_{i}, \mathbf{x} \backslash x_{i}\right) \mu\left(d y_{i} \mid\right.$ $\sigma_{i c}$ ) by taking the expectation conditioned by the $\operatorname{sub} \sigma$-field $\sigma_{i^{c}}$, through the definition of the conditional probability measures, we can give the equivalent expressions for $\mathcal{E}_{i}^{\alpha}(u, v)$ defined by (2.9), $u, v \in \cap_{i \in \mathbb{N}} \mathcal{D}_{i}$, as follows:

$$
\begin{aligned}
\mathcal{E}_{(\alpha)}^{(i)}(u, v) & \equiv \int_{S}\left\{\int_{\mathbb{R} \backslash\left\{x_{i}\right\}} \Phi_{\alpha}\left(u, v ; y_{i}, x_{i}, \mathbf{x} \backslash x_{i}\right) \mu\left(d y_{i} \mid \sigma_{i^{c}}\right)\right\} \mu(d \mathbf{x}) \\
& =\int_{S} \int_{\mathbb{R}}\left\{\int_{\mathbb{R} \backslash\left\{x_{i}\right\}} \Phi_{\alpha}\left(u, v ; y_{i}, x_{i}, \mathbf{x} \backslash x_{i}\right) \mu\left(d y_{i} \mid \sigma_{i^{c}}\right)\right\} \mu\left(d x_{i} \mid \sigma_{i^{c}}\right) \mu(d \mathbf{x}) \\
& =\int_{S}\left\{\int_{\left\{y_{i} \neq y_{i}^{\prime}\right\}} \Phi_{\alpha}\left(u, v ; y_{i}, y_{i}^{\prime}, \mathbf{x} \backslash x_{i}\right) \mu\left(d y_{i} \mid \sigma_{i^{c}}\right) \mu\left(d y_{i}^{\prime} \mid \sigma_{i^{c}}\right)\right\} \mu(d \mathbf{x})
\end{aligned}
$$




$$
=\int_{S \backslash x_{i}}\left\{\int_{\left\{y_{i} \neq y_{i}^{\prime}\right\}} \Phi_{\alpha}\left(u, v ; y_{i}, y_{i}^{\prime}, \mathbf{x} \backslash x_{i}\right) \mu\left(d y_{i} \mid \sigma_{i^{c}}\right) \mu\left(d y_{i}^{\prime} \mid \sigma_{i^{c}}\right)\right\} \mu\left(d\left(\mathbf{x} \backslash x_{i}\right)\right)
$$

where $S \backslash x_{i} \equiv\left\{\left(x_{1}, \ldots, x_{i-1}, x_{i+1}, \ldots\right):\left(x_{1}, \ldots\right) \in S\right\}$ and $\mu\left(d\left(\mathbf{x} \backslash x_{i}\right)\right)$ is the marginal probability distribution of the variable $\mathbf{x} \backslash x_{i}$ (cf. the notation used in (2.8)), i.e., for any $A \in \sigma_{i}$,

$\int_{A} \mu\left(d\left(\mathbf{x} \backslash x_{i}\right)\right)=\int_{S} I_{\mathbb{R}}\left(x_{i}\right) I_{A}\left(\mathbf{x} \backslash x_{i}\right) \mu(d \mathbf{x})$. The third and fourth formulas give the more symmetric definitions for $\mathcal{E}_{i}^{\alpha}(u, v)$ with respect to the variables $y_{i}$ and $x_{i}$ (cf. (1.2.1) of [46]), which will be used in Sect. 4.

Under the adequate assumptions on the density functions $\rho$ (cf. (2.11)), by multiplying adequate weighted functions to $\Phi_{\alpha}$, and then by taking the limit $\alpha \uparrow 2$ for the non-local symmetric form $\mathcal{E}_{(\alpha)}^{(i)}$ given by (2.9), it is possible to define various local Dirichlet forms (cf., e.g., section II-2 of [69]). The corresponding considerations will be announced in forthcoming works.

The following is the main theorem of the closability part of this paper.

Theorem 1. For the symmetric non-local forms $\mathcal{E}_{(\alpha)}, 0<\alpha<2$ given by (2.10) (for $1<\alpha<2$ with the additional assumption (2.11) with (2.12)) the following hold (cf. Remark 1-i), ii)):

(i) $\mathcal{E}_{(\alpha)}$ is well-defined on $\mathcal{F} C_{0}^{\infty}$;

(ii) $\left(\mathcal{E}_{(\alpha)}, \mathcal{F} C_{0}^{\infty}\right)$ is closable in $L^{2}(S ; \mu)$;

(iii) $\left(\mathcal{E}_{(\alpha)}, \mathcal{F} C_{0}^{\infty}\right)$ is Markovian.

Thus, for each $0<\alpha<2$, the closed extension of $\left(\mathcal{E}_{(\alpha)}, \mathcal{F} C_{0}^{\infty}\right)$ denoted by $\left(\mathcal{E}_{(\alpha)}, \mathcal{D}\left(\mathcal{E}_{(\alpha)}\right)\right)$ with the domain $\mathcal{D}\left(\mathcal{E}_{(\alpha)}\right)$, is a non-local Dirichlet form on $L^{2}(S ; \mu)$.

The proof of Theorem 1 is presented in the next section.

\section{Proof of Theorem 1}

We Start the Proof of Theorem 1 for $0<\alpha \leq 1$

For the statement i), we have to show that i-1) for any real valued $\mathcal{B}(S)$-measurable function $u$ on $S$, such that $u=0, \mu-a . e$., it holds that $\mathcal{E}_{(\alpha)}(u, u)=0$ (cf. (3.8) given below), and

i-2) for any $u, v \in \mathcal{F} C_{0}^{\infty}$, there corresponds only one value $\mathcal{E}_{(\alpha)}(u, v) \in \mathbb{R}$, For the statement ii), we have to show the following: For a sequence $\left\{u_{n}\right\}_{n \in \mathbb{N}}, u_{n} \in \mathcal{F} C_{0}^{\infty}$, $n \in \mathbb{N}$, if

$$
\lim _{n \rightarrow \infty}\left\|u_{n}\right\|_{L^{2}(S ; \mu)}=0
$$

and

$$
\lim _{n, m \rightarrow \infty} \mathcal{E}_{(\alpha)}\left(u_{n}-u_{m}, u_{n}-u_{m}\right)=0,
$$

then

$$
\lim _{n \rightarrow \infty} \mathcal{E}_{(\alpha)}\left(u_{n}, u_{n}\right)=0 .
$$


For the statement iii), we have to show that (cf. [46] and Proposition I-4.10 of [69]) for any $\epsilon>0$ there exists a real function $\varphi_{\epsilon}(t),-\infty<t<\infty$, such that $\varphi_{\epsilon}(t)=t, \forall t \in[0,1]$, $-\epsilon \leq \varphi_{\epsilon}(t) \leq 1+\epsilon, \forall t \in(-\infty, \infty)$, and $0 \leq \varphi_{\epsilon}\left(t^{\prime}\right)-\varphi_{\epsilon}(t) \leq t^{\prime}-t$ for $t<t^{\prime}$, such that for any $u \in \mathcal{F} C_{0}^{\infty}$ it holds that $\varphi_{\epsilon}(u) \in \overline{\mathcal{F}} C_{0}^{\infty}$ and

$$
\mathcal{E}_{(\alpha)}\left(\varphi_{\epsilon}(u), \varphi_{\epsilon}(u)\right) \leq \mathcal{E}_{(\alpha)}(u, u) .
$$

i-1) can be seen as follows: For each $i \in \mathbb{N}$ and any real valued $\mathcal{B}(S)$-measurable function $u$, note that for each $\epsilon>0$,

$$
I_{\left\{\epsilon<\left|x_{i}-y_{i}\right|\right\}}\left(y_{i}\right) \Phi_{\alpha}\left(u, u ; y_{i}, x_{i}, \mathbf{x} \backslash x_{i}\right)
$$

defines a $\mathcal{B}(S \times \mathbb{R})$-measurable function. The function $\Phi_{\alpha}\left(u, u ; y_{i}, x_{i}, \mathbf{x} \backslash x_{i}\right)$, is defined by setting $v=u, x=x_{i}$, in (2.8). $\mathcal{B}(S \times \mathbb{R})$ is the Borel $\sigma$-field of $S \times \mathbb{R} . \mathbf{x}=$ $\left(x_{i}, i \in \mathbb{N}\right) \in S$ and $y_{i} \in \mathbb{R}$. Then, $0 \leq I_{\left\{\epsilon<\left|x_{i}-y_{i}\right|\right\}}\left(y_{i}\right) \Phi_{\alpha}\left(u, u ; y_{i}, x_{i}, \mathbf{x} \backslash x_{i}\right)$ converges monotonically to $I_{\left\{y_{i} \neq x_{i}\right\}}\left(y_{i}\right) \Phi_{\alpha}\left(u, u ; y_{i}, x_{i}, \mathbf{x} \backslash x_{i}\right)$ as $\epsilon \downarrow 0$, for every $y_{i} \in \mathbb{R}, \mathbf{x} \in S$, and by Fatou's Lemma, we have

$$
\begin{aligned}
& \int_{S}\left\{\int_{\mathbb{R}} I_{\left\{y_{i} \neq x_{i}\right\}}\left(y_{i}\right) \Phi_{\alpha}\left(u, u ; y_{i}, x_{i}, \mathbf{x} \backslash x_{i}\right) \mu\left(d y_{i} \mid \sigma_{i^{c}}\right)\right\} \mu(d \mathbf{x}) \\
& =\int_{S} \liminf _{\epsilon \downarrow 0}\left\{\int_{\mathbb{R}} I_{\left\{\epsilon<\left|x_{i}-y_{i}\right|\right\}}\left(y_{i}\right) \Phi_{\alpha}\left(u, u ; y_{i}, x_{i}, \mathbf{x} \backslash x_{i}\right) \mu\left(d y_{i} \mid \sigma_{i^{c}}\right)\right\} \mu(d \mathbf{x}) \\
& \leq \liminf _{\epsilon \downarrow 0} \int_{S}\left\{\int_{\mathbb{R}} I_{\left\{\epsilon<\left|x_{i}-y_{i}\right|\right\}}\left(y_{i}\right) \Phi_{\alpha}\left(u, u ; y_{i}, x_{i}, \mathbf{x} \backslash x_{i}\right) \mu\left(d y_{i} \mid \sigma_{i^{c}}\right)\right\} \mu(d \mathbf{x}) .
\end{aligned}
$$

Through the definition of the conditional probability distributions and conditional expectations, we see that, for any $\epsilon>0$,

$$
\begin{aligned}
& \int_{S}\left\{\int_{\mathbb{R}} I_{\left\{\epsilon<\left|x_{i}-y_{i}\right|\right\}}\left(y_{i}\right) \frac{1}{\left|y_{i}-x_{i}\right|^{\alpha+1}}\left(u\left(x_{1}, \ldots, x_{i-1}, y_{i}, x_{i+1}, \ldots\right)\right)^{2} \mu\left(d y_{i} \mid \sigma_{i^{c}}\right)\right\} \mu(d \mathbf{x}) \\
& \leq \frac{1}{\epsilon^{\alpha+1}} \int_{S}\left\{\int_{\mathbb{R}} I_{\left\{\epsilon<\left|x_{i}-y_{i}\right|\right\}}\left(y_{i}\right)\left(u\left(x_{1}, \ldots, x_{i-1}, y_{i}, x_{i+1}, \ldots\right)\right)^{2} \mu\left(d y_{i} \mid \sigma_{i^{c}}\right)\right\} \mu(d \mathbf{x}) \\
& \leq \frac{1}{\epsilon^{\alpha+1}} \int_{S}\left\{\int_{\mathbb{R}}\left(u\left(x_{1}, \ldots, x_{i-1}, y_{i}, x_{i+1}, \ldots\right)\right)^{2} \mu\left(d y_{i} \mid \sigma_{i^{c}}\right)\right\} \mu(d \mathbf{x}) \\
& =\frac{1}{\epsilon^{\alpha+1}} \int_{S}\left(u\left(x_{1}, \ldots, x_{i-1}, x_{i}, x_{i+1}, \ldots\right)\right)^{2} \mu(d \mathbf{x}),
\end{aligned}
$$

and

$$
\begin{aligned}
& \int_{S}\left(u\left(x_{1}, \ldots\right)\right)^{2}\left\{\int_{\mathbb{R}} I_{\left\{\epsilon<\left|x_{i}-y_{i}\right|\right\}}\left(y_{i}\right) \frac{1}{\left|y_{i}-x_{i}\right|^{\alpha+1}} \mu\left(d y_{i} \mid \sigma_{i^{c}}\right)\right\} \mu(d \mathbf{x}) \\
& \leq \frac{1}{\epsilon^{\alpha+1}} \int_{S}\left(u\left(x_{1}, \ldots\right)\right)^{2} \mu(d \mathbf{x}) .
\end{aligned}
$$

From (3.6), by making use of the Cauchy Schwarz inequality

$$
\begin{aligned}
& \mid \int_{S} u\left(x_{1}, \ldots\right)\left\{\int_{\mathbb{R}} I_{\left\{\epsilon<\left|x_{i}-y_{i}\right|\right\}}\left(y_{i}\right) \frac{1}{\left|y_{i}-x_{i}\right|^{\alpha+1}}\right. \\
& \left.\quad \times u\left(x_{1}, \ldots, x_{i-1}, y_{i}, x_{i+1}, \ldots\right) \mu\left(d y_{i} \mid \sigma_{i^{c}}\right)\right\} \mu(d \mathbf{x}) \mid \\
& \quad \leq \frac{1}{\epsilon^{\alpha+1}} \int_{S}\left(u\left(x_{1}, \ldots\right)\right)^{2} \mu(d \mathbf{x}) .
\end{aligned}
$$


By making use of this, (3.6) and (3.7), from (3.5) it follows the property i-1): for all $i \in \mathbb{N}, \mathcal{E}_{(\alpha)}^{(i)}(u, u)=0$, for any real valued $\mathcal{B}(S)$-measurable function $u$ such that $u=0$, $\mu$-a.e.. This then implies

$$
\mathcal{E}_{(\alpha)}(u, u)=0 \quad \text { for all such } u .
$$

In order to prove i-2), for $0<\alpha \leq 1$, take any representative $\tilde{u} \in C_{0}^{\infty}\left(\mathbb{R}^{n}\right)$ of $u \in \mathcal{F} C_{0}^{\infty}, n \in \mathbb{N}$ (precisely, $\tilde{u}$ is defined as (3.15)). Since $\tilde{u}$ is a Lipschitz function, it is easy to see from the definition (2.8) that (cf. the formula on $\left|\eta_{M, i}(x)-\eta_{M, i}(y)\right|^{2}$ given above (4.33), in the next section) there exists an $M<\infty$ depending on $\tilde{u}$ such that

$$
0 \leq \Phi_{\alpha}\left(\tilde{u}, \tilde{u} ; y_{i}, y_{i}^{\prime}, \mathbf{x} \backslash x_{i}\right) \leq M, \quad \forall \mathbf{x} \in S, \text { and } \forall y_{i}, y_{i}^{\prime} \in \mathbb{R} .
$$

By this, we have that

$$
\mathcal{E}_{(\alpha)}^{(i)}(\tilde{u}, \tilde{u}) \in \mathbb{R}
$$

(in fact, for only a finite number of $i \in \mathbb{N}$. we have $\mathcal{E}_{(\alpha)}^{(i)}(\tilde{u}, \tilde{u}) \neq 0$, cf. also (2.10)). Since, $u=\tilde{u}+\overline{0}$ for some real valued $\mathcal{B}(S)$-measurable function $\overline{0}$ such that $\overline{0}=0$, $\mu$-a.e., by (3.9) together with i-1) (cf. (3.8)) and the Cauchy-Schwarz inequality, for $u \in \mathcal{F} C_{0}^{\infty}$, $\mathcal{E}_{(\alpha)}(u, u)=\mathcal{E}_{(\alpha)}(\tilde{u}, \tilde{u}) \in \mathbb{R}, 0<\alpha \leq 1$. Thus $\mathcal{E}_{(\alpha)}(u, u)$ is uniquely defined. Then by the Cauchy-Schwarz inequality i-2) follows.

ii) can be proved as follows (cf. section 1 of [46]): Suppose that a sequence $\left\{u_{n}\right\}_{n \in \mathbb{N}}$ satisfies (3.1) and (3.2). Then, by (3.1) there exists a measurable set $\mathcal{N} \in \mathcal{B}(S)$ and a sub sequence $\left\{u_{n_{k}}\right\}$ of $\left\{u_{n}\right\}$ such that

$$
\mu(\mathcal{N})=0, \quad \lim _{n_{k} \rightarrow \infty} u_{n_{k}}(\mathbf{x})=0, \quad \forall \mathbf{x} \in S \backslash \mathcal{N} .
$$

Define

$$
\tilde{u}_{n_{k}}(\mathbf{x})=u_{n_{k}}(\mathbf{x}) \quad \text { for } \mathbf{x} \in S \backslash \mathcal{N}, \quad \text { and } \quad \tilde{u}_{n_{k}}(\mathbf{x})=0 \quad \text { for } \mathbf{x} \in \mathcal{N} .
$$

Then,

$$
\tilde{u}_{n_{k}}(\mathbf{x})=u_{n_{k}}(\mathbf{x}), \quad \mu-\text { a.e. }, \quad \lim _{n_{k} \rightarrow \infty} \tilde{u}_{n_{k}}(\mathbf{x})=0, \quad \forall \mathbf{x} \in S .
$$

By the fact $\mathrm{i}-1)$, precisely by (3.8) shown above and (3.10), for each $i$, we see that

$$
\begin{aligned}
& \int_{S}\left\{\int_{\mathbb{R}} I_{\left\{y_{i} \neq x_{i}\right\}}\left(y_{i}\right) \Phi_{\alpha}\left(u_{n}, u_{n} ; y_{i}, x_{i}, \mathbf{x} \backslash x_{i}\right) \mu\left(d y_{i} \mid \sigma_{i^{c}}\right)\right\} \mu(d \mathbf{x}) \\
& \quad=\int_{S}\left\{\int_{\mathbb{R}} I_{\left\{y_{i} \neq x_{i}\right\}}\left(y_{i}\right) \lim _{n_{k} \rightarrow \infty} \Phi_{\alpha}\left(u_{n}-\tilde{u}_{n_{k}}, u_{n}-\tilde{u}_{n_{k}} ; y_{i}, x_{i}, \mathbf{x} \backslash x_{i}\right) \mu\left(d y_{i} \mid \sigma_{i^{c}}\right)\right\} \mu(d \mathbf{x}) \\
& \quad \leq \liminf _{n_{k} \rightarrow \infty} \int_{S}\left\{\int_{\mathbb{R}} I_{\left\{y_{i} \neq x_{i}\right\}} \Phi_{\alpha}\left(u_{n}-\tilde{u}_{n_{k}}, u_{n}-\tilde{u}_{n_{k}} ; y_{i}, x_{i}, \mathbf{x} \backslash x_{i}\right) \mu\left(d y_{i} \mid \sigma_{i^{c}}\right)\right\} \mu(d \mathbf{x}) \\
& =\liminf _{n_{k} \rightarrow \infty} \int_{S}\left\{\int_{\mathbb{R}} I_{\left\{y_{i} \neq x_{i}\right\}} \Phi_{\alpha}\left(u_{n}-u_{n_{k}}, u_{n}-u_{n_{k}} ; y_{i}, x_{i}, \mathbf{x} \backslash x_{i}\right) \mu\left(d y_{i} \mid \sigma_{i^{c}}\right)\right\} \mu(d \mathbf{x}) \\
& \equiv \liminf _{n_{k} \rightarrow \infty} \mathcal{E}_{(\alpha)}^{(i)}\left(u_{n}-u_{n_{k}}, u_{n}-u_{n_{k}}\right) .
\end{aligned}
$$

Now, by applying the assumption (3.2) to the right hand side of (3.11), we get

$$
\lim _{n \rightarrow \infty} \mathcal{E}_{(\alpha)}^{(i)}\left(u_{n}, u_{n}\right)=0, \quad \forall i \in \mathbb{N} .
$$


(3.12) together with i) shows that for each $i \in \mathbb{N}, \mathcal{E}_{(\alpha)}^{(i)}$ with the domain $\mathcal{F} C_{0}^{\infty}$ is closable in $L^{2}(S ; \mu)$. Since, $\mathcal{E}_{(\alpha)} \equiv \sum_{i \in \mathbb{N}} \mathcal{E}_{(\alpha)}^{(i)}$, by using Fatou's Lemma, from (3.12) and the assumption (3.2) we see that

$$
\begin{aligned}
& \mathcal{E}_{(\alpha)}\left(u_{n}, u_{n}\right)=\sum_{i \in \mathbb{N}} \lim _{m \rightarrow \infty} \mathcal{E}_{(\alpha)}^{(i)}\left(u_{n}-u_{m}, u_{n}-u_{m}\right) \\
& \leq \liminf _{m \rightarrow \infty} \mathcal{E}_{(\alpha)}\left(u_{n}-u_{m}, u_{n}-u_{m}\right) \rightarrow 0,
\end{aligned}
$$

as $n \rightarrow \infty$. This proves (3.3) (cf. Proposition I-3.7 of [69] for a general argument). The proof of ii) is thus complete.

iii) can be shown as follows: For each $\epsilon>0$, take a smooth function $\varphi_{\epsilon}(t)$ such that $\varphi_{\epsilon}(t)=t$ for $t \in\left[-\frac{\epsilon}{2}, 1+\frac{\epsilon}{2}\right], \varphi_{\epsilon}(t)=-\epsilon$ for $t \leq-2 \epsilon, \varphi_{\epsilon}(t)=1+\epsilon$ for $t \geq 1+2 \epsilon$, and it satisfies $0 \leq \varphi_{\epsilon}\left(t^{\prime}\right)-\varphi_{\epsilon}(t) \leq t^{\prime}-t$ for $\left.t<t^{\prime}\right)$. Then, for $u \in \mathcal{F} C_{0}^{\infty} \subset \mathcal{D}\left(\mathcal{E}_{(\alpha)}\right)$, it holds that $\varphi_{\epsilon}(u(\mathbf{x})) \in \mathcal{F} C_{0}^{\infty} \subset \mathcal{D}\left(\mathcal{E}_{(\alpha)}\right)$, and (3.4) is satisfied (cf. section 1 of [46]]).

For $0<\alpha \leq 1$ the proof of Theorem 1 is therefore complete.

The proof of Theorem 1, for $1<\alpha<2$

The proof of i-1), ii) and iii) can be carried out by exactly the same manner as the previous proof we have provided for the case $0<\alpha \leq 1$. We only show that i-2), i.e., $\mathcal{E}_{(\alpha)}(u, u)<\infty, \forall u \in \mathcal{F} C_{0}^{\infty}$ also holds when we make use of the additional assumption (2.11) with (2.12), i.e., of existence of a locally bounded probability density (cf. (2.12)), $\rho\left(y \mid \sigma_{i^{c}}\right)$ for $\mu\left(d y \mid \sigma_{i^{c}}\right)$, i.e., $\mu\left(d y \mid \sigma_{i^{c}}\right)=\rho\left(y \mid \sigma_{i^{c}}\right) d y, \mu-a . e .$. Under this assumption by applying Young's inequality, we derive i-2) (cf. the formula given above (3.9), also cf. (3.6), (3.7) and (3.8)) as follows: For $1<\alpha<2$, by the definition (2.8), we note that

$$
\Phi_{\alpha}\left(u, u ; y_{i}, x_{i}, \mathbf{x} \backslash x_{i}\right)=\Phi_{1}\left(u, u ; y_{i}, x_{i}, \mathbf{x} \backslash x_{i}\right) \cdot \frac{1}{\left|x_{i}-y_{i}\right|^{\alpha-1}} .
$$

Take a representative $\tilde{u} \in C_{0}^{\infty}\left(\mathbb{R}^{n}\right)$ of $u \in \mathcal{F} C_{0}^{\infty}$, given by (3.15) below, and let $I_{K_{i}}$ be the indicator function for a compact set $K_{i}$ such that

$$
K_{i} \equiv\left\{x \in \mathbb{R}: x=x^{\prime}-x^{\prime \prime}, x^{\prime}, x^{\prime \prime} \in U_{i}\right\},
$$

with

$$
U_{i} \equiv \text { the closure of }\left\{x_{i} \in \mathbb{R}: f\left(x_{1}, \ldots, x_{i-1}, x_{i}, x_{i+1}, \ldots x_{n}\right) \neq 0\right\}, i=1, \ldots, n,
$$

where

$$
f \in C_{0}^{\infty}\left(\mathbb{R}^{n} \rightarrow \mathbb{R}\right), \quad \text { such that } \tilde{u}(\mathbf{x})=f \cdot \prod_{j \in \mathbb{N}} I_{\mathbb{R}}\left(x_{j}\right) \in \mathcal{F} C_{0}^{\infty}
$$

Also by the assumption (2.12), the probability density satisfies the following: for each $i \in \mathbb{N}$ and the compact $U_{i}$, there exists an $0<L_{U_{i}, i}<\infty$, and for a.e. $y \in \mathbb{R}$, with respect to the Lebesgue measure,

$$
I_{U_{i}, i}(y) \rho\left(y \mid \sigma_{i^{c}}\right) \leq L_{U_{i}, i}, \quad \mu-a . e . .
$$

Hence by Young's inequality (for the convolutions) we have the following bound: for $i=1, \ldots, n$, 


$$
\sup _{x \in \mathbb{R}}\left|\int_{\mathbb{R}} \frac{I_{K_{i}}(x-y)}{|x-y|^{\alpha-1}} I_{U_{i}}(y) \rho\left(y \mid \sigma_{i}^{c}\right) d y\right|=L_{U_{i}, i}\left\|\frac{I_{K_{i}}}{|\cdot|^{\alpha-1}}\right\|_{L^{1}(\mathbb{R})}, \quad \mu-\text { a.e.. }
$$

For notational simplicity, let $f\left(x_{1}, \ldots, x_{i-1}, x_{i}, x_{i+1}, \ldots, x_{n}\right)$ be denoted by $f\left(\cdot, x_{i}, \cdot\right)$. Since, $f\left(\cdot, x_{i}, \cdot\right)=f\left(\cdot, y_{i}, \cdot\right)=0$ for $x_{i} \in U_{i}^{c}$ and $y_{i} \in U_{i}^{c}$, we note that the following is true:

$$
\left(f\left(\cdot, x_{i}, \cdot\right)-f\left(\cdot, y_{i}, \cdot\right)\right) I_{U_{i}}^{c}\left(x_{i}\right) I_{U_{i}}^{c}\left(y_{i}\right)=0 .
$$

We then have the following evaluation for $x_{i} \neq y_{i}$ :

$$
\begin{aligned}
I_{K_{i}} & \left(x_{i}-y_{i}\right) \frac{\left(f\left(\cdot, x_{i}, \cdot\right)-f\left(\cdot, y_{i}, \cdot\right)\right)^{2}}{\left|x_{i}-y_{i}\right|^{\alpha+1}} \\
= & I_{K_{i}}\left(x_{i}-y_{i}\right) \frac{\left(f\left(\cdot, x_{i}, \cdot\right)-f\left(\cdot, y_{i}, \cdot\right)\right)^{2}}{\left|x_{i}-y_{i}\right|^{\alpha+1}} \\
& \times\left\{I_{U_{i}}\left(x_{i}\right) I_{U_{i}}\left(y_{i}\right)+I_{U_{i}^{c}}\left(x_{i}\right) I_{U_{i}}\left(y_{i}\right)+I_{U_{i}}\left(x_{i}\right) I_{U_{i}^{c}}\left(y_{i}\right)+I_{U_{i}^{c}}\left(x_{i}\right) I_{U_{i}^{c}}\left(y_{i}\right)\right\} \\
= & I_{K_{i}}\left(x_{i}-y_{i}\right) \frac{\left(f\left(\cdot, x_{i}, \cdot\right)-f\left(\cdot, y_{i}, \cdot\right)\right)^{2}}{\left|x_{i}-y_{i}\right|^{2}} \frac{1}{\left|x_{i}-y_{i}\right|^{\alpha-1}} \\
& \times\left\{I_{U_{i}}\left(x_{i}\right) I_{U_{i}}\left(y_{i}\right)+I_{U_{i}^{c}}\left(x_{i}\right) I_{U_{i}}\left(y_{i}\right)+I_{U_{i}}\left(x_{i}\right) I_{U_{i}^{c}}\left(y_{i}\right)\right\} \\
\leq & \left(\sup _{0<\theta<1}\left|f_{x_{i}}\left(\cdot, x_{i}+\theta\left(y_{i}-x_{i}\right), \cdot\right)\right|^{2}\right) \frac{I_{K_{i}}\left(x_{i}-y_{i}\right)}{\left|x_{i}-y_{i}\right|^{\alpha-1}} \\
& \times\left\{I_{U_{i}}\left(x_{i}\right) I_{U_{i}}\left(y_{i}\right)+I_{U_{i}^{c}}\left(x_{i}\right) I_{U_{i}}\left(y_{i}\right)+I_{U_{i}}\left(x_{i}\right) I_{U_{i}^{c}}\left(y_{i}\right)\right\},
\end{aligned}
$$

in the above and below we denoted $\frac{\partial f}{\partial x_{i}}=f_{x_{i}}$. From (3.17), by making use of (3.16) we have that

$$
\begin{aligned}
& \int_{S}\left\{\int_{x_{i} \neq y_{i}} I_{K_{i}}\left(x_{i}-y_{i}\right) \frac{\left(f\left(\cdot, x_{i}, \cdot\right)-f\left(\cdot, y_{i}, \cdot\right)\right)^{2}}{\left|x_{i}-y_{i}\right|^{\alpha+1}} \rho\left(x_{i} \mid \sigma_{i}^{c}\right) \rho\left(y_{i} \mid \sigma_{i}^{c}\right) d x_{i} d y_{i}\right\} \mu(d \mathbf{x}) \\
& \quad \leq 3\left(\sup _{0<\theta<1}\left|f_{x_{i}}\left(\cdot, x_{i}+\theta\left(y_{i}-x_{i}\right), \cdot\right)\right|^{2}\right)\left\{\int_{S}\left\{\int_{\mathbb{R}} L_{U_{i}, i}\left\|\frac{I_{K_{i}}}{|\cdot|^{\alpha-1}}\right\|_{L^{1}(\mathbb{R})} \rho\left(x_{i} \mid \sigma_{i}^{c}\right) d x_{i}\right\} \mu(d \mathbf{x})\right\} \\
& \quad=3\left(\sup _{0<\theta<1}\left|f_{x_{i}}\left(\cdot, x_{i}+\theta\left(y_{i}-x_{i}\right), \cdot\right)\right|^{2}\right) L_{U_{i}, i}\left\|\frac{I_{K_{i}}}{|\cdot|^{\alpha-1}}\right\|_{L^{1}(\mathbb{R})} .
\end{aligned}
$$

Moreover, for $\tilde{u} \in \mathcal{F} C_{0}^{\infty}$ given by (3.15) with $f$ satisfying $f \neq 0$, since $f \in$ $C_{0}^{\infty}\left(\mathbb{R}^{n} \rightarrow \mathbb{R}\right)$ for some $n \in \mathbb{N}$, for each $i=1, \ldots, n$, there exists $m_{i}>0$ and it holds that $K_{i}^{c} \subset\left\{x:|x| \geq m_{i}\right\} \subset \mathbb{R}$. Hence for each $1<\alpha<2$ and $i=1, \ldots, n$, there exists an $M_{i}^{\prime}<\infty$ such that

$$
\frac{1}{\left|x_{i}-y_{i}\right|^{\alpha-1}} I_{K_{i}^{c}}\left(x_{i}-y_{i}\right) \leq M_{i}^{\prime}, \quad \forall x_{i}, y_{i} \in \mathbb{R} .
$$

In addition, by the definition (2.8), for each $\tilde{u} \in \mathcal{F} C_{0}^{\infty}$ given by (3.15), there exists an $M<\infty$ (cf. the formula given above (3.9)) and

$$
0 \leq \Phi_{1}\left(\tilde{u}, \tilde{u} ; y_{i}, y_{i}^{\prime}, \mathbf{x} \backslash x_{i}\right) \leq M, \quad \forall \mathbf{x} \in S, \text { and } \forall y_{i}, y_{i}^{\prime} \in \mathbb{R}, i=1, \ldots, n .
$$


By making use of this and (3.19), with (3.13), we also have that (cf. (3.18))

$$
\begin{aligned}
& \int_{S}\left\{\int_{x_{i} \neq y_{i}} I_{K_{i}^{c}}\left(x_{i}-y_{i}\right) \frac{\left(f\left(\cdot, x_{i}, \cdot\right)-f\left(\cdot, y_{i}, \cdot\right)\right)^{2}}{\left|x_{i}-y_{i}\right|^{\alpha+1}} \rho\left(x_{i} \mid \sigma_{i}^{c}\right) \rho\left(y_{i} \mid \sigma_{i}^{c}\right) d x_{i} d y_{i}\right\} \mu(d \mathbf{x}) \\
& \quad \leq M \cdot M_{i}^{\prime} .
\end{aligned}
$$

From (3.20) and (3.18), by the symmetric expression (2.13) we see that $\mathcal{E}_{(\alpha)}^{(i)}(\tilde{u}, \tilde{u})<\infty$, $\forall i \in \mathbb{N}$, (cf. (4.64) in Sect. 4, for similar detailed evaluation) and $\mathcal{E}_{(\alpha)}(\tilde{u}, \tilde{u})<\infty$ (in fact, for only a finite number of $i \in \mathbb{N}, \mathcal{E}_{(\alpha)}^{(i)}(\tilde{u}, \tilde{u}) \neq 0$, cf. also (2.10)) for any $\tilde{u} \in \mathcal{F} C_{0}^{\infty}$ given by (3.15). Since i-1) holds for $1<\alpha<2$, passing through the same argument as for the case where $0<\alpha \leq 1$, we have i-2) for $1<\alpha<2$.

Remark 3. (i) The condition (2.12) can be substituted by a general abstract condition that is an analogue of (2.1) of [49] given for the finite dimensional cases.

(ii) For each $0 \leq \alpha<2$, by using the formulas (2.9), if we define a quadratic form $\overline{\mathcal{E}}_{(\alpha)}$ on $L^{2}(S ; \mu)$ (cf. (2.10)) such that

$$
\overline{\mathcal{E}}_{(\alpha)}(u, v) \equiv \sum_{i \in \mathbb{N}} \mathcal{E}_{(\alpha)}^{(i)}(u, v), \quad u, v \in \mathcal{D}\left(\overline{\mathcal{E}}_{(\alpha)}\right),
$$

where $\mathcal{D}\left(\overline{\mathcal{E}}_{(\alpha)}\right)$ is the domain of $\overline{\mathcal{E}}_{(\alpha)}$ defined by

$$
\mathcal{D}\left(\overline{\mathcal{E}}_{(\alpha)}\right) \equiv\left\{u \in L^{2}(S ; \mu): \overline{\mathcal{E}}_{(\alpha)}(u, u)<\infty\right\}
$$

then, by the same arguments as for the proof of Theorem 1, it is possible to see that $\overline{\mathcal{E}}_{(\alpha)}$ with the domain $\mathcal{D}\left(\overline{\mathcal{E}}_{(\alpha)}\right)$ on $L^{2}(S ; \mu)$ is a closed form. But it is not guaranteed that $\mathcal{D}\left(\overline{\mathcal{E}}_{(\alpha)}\right)$ includes $\mathcal{F} C_{0}^{\infty}$ as its dense subset, and hence for $\overline{\mathcal{E}}_{(\alpha)}$, the Markovian property (cf. Theorem 1-ii) and (3.1)) and the quasi regularity (cf. next section) can not be discussed through a standard argument (cf. e.g., section 1.2 of [46]). Moreover it may happen that $\mathcal{D}\left(\overline{\mathcal{E}}_{(\alpha)}\right)=\{0\}$, where 0 is the $\mu$ equivalent class of measurable functions $f=0, \mu-a . e$.

\section{Strict Quasi-regularity}

In this section, we give sufficient conditions (cf. Theorem 2 and Theorem 3 below) under which the Dirichlet forms (i.e. the closed Markovian symmetric forms) defined in the previous section are strictly quasi-regular (cf., [29-31] and section IV-3 of [69]).

Denote by $\left(\mathcal{E}_{(\alpha)}, \mathcal{D}\left(\mathcal{E}_{(\alpha)}\right)\right)$ the Dirichlet form on $L^{2}(S ; \mu)$, with the domain $\left.\mathcal{D}\left(\mathcal{E}_{(\alpha)}\right)\right)$ defined through Theorem 1 in the previous section, obtained as the closed extension of the closable Markovian symmetric form $\mathcal{E}_{(\alpha)}$ of its restriction to $\mathcal{F} C_{0}^{\infty}$. We shall use the same notation $\mathcal{E}_{(\alpha)}$ for the closable form and the closed form.

Recall that the state space $S$ is taken to be a Fréchet space, either a weighted $l^{p}$ space, $l_{\left(\beta_{i}\right)}^{p}$ defined by (2.1), or a weighted $l^{\infty}$ space, $l_{\left(\beta_{i}\right)}^{\infty}$ defined by (2.2), or the direct product space $\mathbb{R}^{\mathbb{N}}$ defined by (2.3).

For each $i \in \mathbb{N}$, we denote by $X_{i}$ the random variable (i.e., measurable function) on $(S, \mathcal{B}(S), \mu)$, that represents the coordinate $x_{i}$ of $\mathbf{x}=\left(x_{1}, x_{2}, \ldots\right)$, precisely,

$$
X_{i}: S \ni \mathbf{x} \longmapsto x_{i} \in \mathbb{R} .
$$


By making use of the random variable $X_{i}$, we have the following probabilistic expression:

$$
\int_{S} I_{B}\left(x_{i}\right) \mu(d \mathbf{x})=\mu\left(X_{i} \in B\right), \quad \text { for } B \in \mathcal{B}(S)
$$

Theorem 2. Let $0<\alpha \leq 1$, and $\left(\mathcal{E}_{(\alpha)}, \mathcal{D}\left(\mathcal{E}_{(\alpha)}\right)\right)$ be the closed Markovian symmetric form defined through Theorem 1.

(i) In the case where $S=l_{\left(\beta_{i}\right)}^{p}$, for some $1 \leq p<\infty$, as defined by (2.1), if there exists a positive sequence $\left\{\gamma_{i}\right\}_{i \in \mathbb{N}}$ such that $\sum_{i=1}^{\infty} \gamma_{i}^{-1}<\infty$ (i.e., $\left\{\gamma_{i}^{-\frac{1}{p}}\right\}_{i \in \mathbb{N}}$ is a positive $l^{p}$ sequence), and an $M_{0} \in(0, \infty)$, and both

$$
\begin{aligned}
& \sum_{i=1}^{\infty}\left(\beta_{i} \gamma_{i}\right)^{\frac{\alpha+1}{p}} \cdot \mu\left(\beta_{i}^{\frac{1}{p}}\left|X_{i}\right|>M_{0} \cdot \gamma_{i}^{-\frac{1}{p}}\right)<\infty, \\
& \mu\left(\bigcup_{M \in \mathbb{N}}\left\{\left|X_{i}\right| \leq M \cdot \beta_{i}^{-\frac{1}{p}} \gamma_{i}^{-\frac{1}{p}}, \forall i \in \mathbb{N}\right\}\right)=1,
\end{aligned}
$$

hold, then $\left(\mathcal{E}_{(\alpha)}, \mathcal{D}\left(\mathcal{E}_{(\alpha)}\right)\right)$ is a quasi-regular Dirichlet form.

(ii) In the case where $S=l_{\left(\beta_{i}\right)}^{\infty}$ as defined by (2.2), if there exists a sequence $\left\{\gamma_{i}\right\}_{i \in \mathbb{N}}$ such that $0<\gamma_{1} \leq \gamma_{2} \leq \cdots \rightarrow \infty$, and an $M_{0} \in(0, \infty)$, and both

$$
\begin{aligned}
& \sum_{i=1}^{\infty}\left(\beta_{i} \gamma_{i}\right)^{\alpha+1} \cdot \mu\left(\beta_{i}\left|X_{i}\right|>M_{0} \cdot \gamma_{i}^{-1}\right)<\infty, \\
& \mu\left(\bigcup_{M \in \mathbb{N}}\left\{\left|X_{i}\right| \leq M \cdot \beta_{i}^{-1} \gamma_{i}^{-1}, \forall i \in \mathbb{N}\right\}\right)=1,
\end{aligned}
$$

hold, then $\left(\mathcal{E}_{(\alpha)}, \mathcal{D}\left(\mathcal{E}_{(\alpha)}\right)\right)$ is a quasi-regular Dirichlet form.

(iii) In the case where $S=\mathbb{R}^{\mathbb{N}}$ as defined by $(2.3),\left(\mathcal{E}_{(\alpha)}, \mathcal{D}\left(\mathcal{E}_{(\alpha)}\right)\right)$ is a quasi-regular Dirichlet form.

(iv) The forms $\left(\mathcal{E}_{(\alpha)}, \mathcal{D}\left(\mathcal{E}_{(\alpha)}\right)\right)$ in the statements $\left.i\right)$, ii), iii) are strictly quasi-regular Dirichlet forms.

Remark 4. (i) Generally, for a real valued random variable $X$ on a probability space $(\Omega, \mathcal{F}, P)$ (denoting the expectations with respect to $P$ by $E[\cdot])$, for $1 \leq r<\infty$, the following Cebysev type inequality holds:

$$
P(|X|>K))<\frac{E\left[|X|^{r}\right]}{K^{r}}
$$

Thus, by denoting the expectations (i.e. the integrations) with respect to the measure $\mu$ by $E_{\mu}[\cdot]$, we see that the following inequality is a sufficient condition for (4.3):

$$
\sum_{i=1}^{\infty} E_{\mu}\left[\left|X_{i}\right|^{2}\right] \cdot\left(\beta_{i} \gamma_{i}\right)^{\frac{2(\alpha+1)}{p}}<\infty
$$

Similarly, by (4.7), we see that the following inequality is a sufficient condition for (4.5):

$$
\sum_{i=1}^{\infty} E_{\mu}\left[\left|X_{i}\right|^{2}\right]\left(\beta_{i} \gamma_{i}\right)^{2(\alpha+1)}<\infty
$$


(ii) In the case where $S=l_{\left(\beta_{i}\right)}^{2}$, if the probability mesure $\mu$ is constructed through the Bochner-Minlos theorem, then the support property (4.4) of $\mu$ can be discussed as part of the statements of the same theorem (cf. Theorem 5 and Examples in Sect. 5).

(iii) To prove Theorem 2 , for each $f \in C_{0}^{\infty}\left(\mathbb{R}^{n} \rightarrow \mathbb{R}\right.$ ) (for some $n \in \mathbb{N}$ ), the functions $u(\mathbf{x}) \equiv f\left(x_{1}, \ldots, x_{n}\right) \prod_{i>1} I_{\mathbb{R}}\left(x_{i}\right), f_{M} \in \mathcal{D}\left(\mathcal{E}_{(\alpha)}\right)$ (see (4.43) below), which is a function having a compact support in $S$ defined by (4.27) below, and $f_{M, k}$ defined by (4.36) below, which is a continuos function on $S$, play the crucial roles. For the infinite dimensional topological vector spaces $S$, any compact set in $S$ can not have an interior (cf., e.g., Theorem 9.2 in [91]), and hence $f_{M}$ is not a continuous function on $S$, but under the condition (4.4) or (4.6) it will be proven that $f_{M} \in \mathcal{D}\left(\mathcal{E}_{(\alpha)}\right)$ and $f_{M}$ approximates $u$ arbitraly with respect to the norm $\|\cdot\|_{L^{2}(S ; \mu)}+\sqrt{\mathcal{E}_{(\alpha)}}$ as $M \rightarrow \infty$ (see (4.43) and (4.46)).

We prepare the following Lemma 1, that is the Lemma 2.12 in section I-2 of [69], by which the proof of Theorem 2 will follow. Here we quote it in a simplified way that is adapted to the present paper:

Lemma 1. For the closed form $\left(\mathcal{E}_{(\alpha)}, \mathcal{D}\left(\mathcal{E}_{(\alpha)}\right)\right.$ with the domain $\mathcal{D}\left(\mathcal{E}_{(\alpha)}\right)$ that is the closure of $\mathcal{F} C_{0}^{\infty}$, a dense subset of $L^{2}(S ; \mu)$ (cf. Remark 1$)$, defined by Theorem 1 , the following holds: If a sequence $\left\{u_{n}\right\}_{n \in \mathbb{N}}, u_{n} \in \mathcal{D}\left(\mathcal{E}_{(\alpha)}\right), n \in \mathbb{N}$ satisfies

$$
\sup _{n \in \mathbb{N}} \mathcal{E}_{(\alpha)}\left(u_{n}, u_{n}\right)<\infty, \quad \text { and } \quad \lim _{n \rightarrow \infty} u_{n}=u \text {, in } L^{2}(S ; \mu),
$$

then

$$
u \in \mathcal{D}\left(\mathcal{E}_{(\alpha)}\right) \quad \text { and } \quad \mathcal{E}_{(\alpha)}(u, u) \leq \liminf _{n \rightarrow \infty} \mathcal{E}_{(\alpha)}\left(u_{n}, u_{n}\right) .
$$

Moreover, there exists a subsequence $\left\{u_{n_{k}}\right\}_{k \in \mathbb{N}}$ of $\left\{u_{n}\right\}_{n \in \mathbb{N}}$ such that its Cesàro mean

$$
\begin{aligned}
& w_{n} \equiv \frac{1}{n} \sum_{k=1}^{n} u_{n_{k}} \rightarrow u \text { in } \mathcal{D}\left(\mathcal{E}_{(\alpha)}\right) \\
& \text { with respect to the norm }\|\cdot\|_{L^{2}(S ; \mu)}+\sqrt{\mathcal{E}_{(\alpha)}} \text { as } n \rightarrow \infty .
\end{aligned}
$$

Proof of Theorem 2. We have to verify that the Dirichlet forms $\left(\mathcal{E}_{(\alpha)}, \mathcal{D}\left(\mathcal{E}_{(\alpha)}\right)\right)$ satisfy the definition of quasi-regularity given by Definition 3.1 in section IV-3 of [69]. Namely, by using the same notions as in [69], we have to certify that the following i), ii) and iii) are satisfied by $\left(\mathcal{E}_{(\alpha)}, \mathcal{D}\left(\mathcal{E}_{(\alpha)}\right)\right)$ :

(i) There exists an $\mathcal{E}_{(\alpha)}$-nest $\left(D_{M}\right)_{M \in \mathbb{N}}$ consisting of compact sets.

(ii) There exists a subset of $\mathcal{D}\left(\mathcal{E}_{(\alpha)}\right)$, that is dense with respect to the norm $\|\cdot\|_{L^{2}(S ; \mu)}+\sqrt{\mathcal{E}_{(\alpha)}}$, and the elements of the subset have $\mathcal{E}_{(\alpha)}$-quasi continuous versions.

(iii) There exists $u_{n} \in \mathcal{D}\left(\mathcal{E}_{(\alpha)}\right), n \in \mathbb{N}$, having $\mathcal{E}_{(\alpha)}$-quasi continuous $\mu$-versions $\tilde{u}_{n}$, $n \in \mathbb{N}$, and an $\mathcal{E}_{(\alpha)}$-exceptional set $\mathcal{N} \subset S$ such that $\left\{\tilde{u}_{n}: n \in \mathbb{N}\right\}$ separates the points of $S \backslash \mathcal{N}$.

Also, the fact that the quasi-regular Dirichlet form $\left(\mathcal{E}_{(\alpha)}, \mathcal{D}\left(\mathcal{E}_{(\alpha)}\right)\right)$ is looked upon as a strictly quasi-regular Dirichlet form can be guaranteed by showing the following (see Proposition V-2.15 of [69]):

(iv) $1 \in \mathcal{D}\left(\mathcal{E}_{(\alpha)}\right)$. 
By Remark 1 and Theorem 1 in Sect. 2, the above ii) and iii) hold for $\left(\mathcal{E}_{(\alpha)}, \mathcal{D}\left(\mathcal{E}_{(\alpha)}\right)\right)$ : since $\mathcal{F} C_{0}^{\infty} \subset C(S \rightarrow \mathbb{R})$ by Remark 1-ii) and $\mathcal{D}\left(\mathcal{E}_{(\alpha)}\right)$ is the closure of $\mathcal{F} C_{0}^{\infty}$ by Theorem 1, we can take $\mathcal{F} C_{0}^{\infty}$ as the subset of $\mathcal{D}\left(\mathcal{E}_{(\alpha)}\right)$ mentioned in the above ii), also since $\mathcal{F} C_{0}^{\infty}$ separates the points $S$, we see that the above iii) holds.

Hence, we have only to show that the above i) and iv) hold for $\left(\mathcal{E}_{(\alpha)}, \mathcal{D}\left(\mathcal{E}_{(\alpha)}\right)\right)$.

We start the proof of i). Equivalently (cf. Definition 2.1. in section III-2 of [69]), we have to show that there exists an increasing sequence $\left(D_{M}\right)_{M \in \mathbb{N}}$ of compact subsets of $S$ such that $\cup_{m \geq 1} \mathcal{D}\left(\mathcal{E}_{(\alpha)}\right)_{D_{M}}$ is dense in $\mathcal{D}\left(\mathcal{E}_{(\alpha)}\right)$ (with respect to the norm $\left.\|\cdot\|_{L^{2}(S ; \mu)}+\sqrt{\mathcal{E}_{(\alpha)}}\right)$, where $\mathcal{D}\left(\mathcal{E}_{(\alpha)}\right)_{D_{M}}$ is the subspace of $\mathcal{D}\left(\mathcal{E}_{(\alpha)}\right)$ the elements of which are functions with supports contained in $D_{M}$. For this, by Theorem 1 , since $\mathcal{D}\left(\mathcal{E}_{(\alpha)}\right)$ is the closure of $\mathcal{F} C_{0}^{\infty}$, it suffices to show the following: there exists a sequence of compact sets

$$
D_{M} \subset S, \quad M \in \mathbb{N}
$$

and a subset $\tilde{\mathcal{D}}\left(\mathcal{E}_{(\alpha)}\right) \subset L^{2}(S ; \mu)$ that satisfies

$$
\tilde{\mathcal{D}}\left(\mathcal{E}_{(\alpha)}\right) \subset \bigcup_{M \geq 1} \mathcal{D}\left(\mathcal{E}_{(\alpha)}\right)_{D_{M}} ;
$$

and, moreover, for any $u \in \mathcal{F} C_{0}^{\infty}$ there exists a sequence $\left\{u_{n}\right\}_{n \in \mathbb{N}}, u_{n} \in \tilde{\mathcal{D}}\left(\mathcal{E}_{(\alpha)}\right), n \in \mathbb{N}$, such that

$$
\lim _{n \rightarrow \infty} u_{n}=u \text { in } \mathcal{D}\left(\mathcal{E}_{(\alpha)}\right)
$$

with respect to the norm $\|\cdot\|_{L^{2}(S ; \mu)}+\sqrt{\mathcal{E}_{(\alpha)}}$.

Proof of (4.13) and (4.14) for $S=l_{\left(\beta_{i}\right)}^{p}, 1 \leq p<\infty$. We start from the proof of (4.13) and (4.14) by considering a suitable system $D_{M}, M \in \mathbb{N}$, of compact sets Let $1 \leq p<\infty$ be fixed. For each $M \in \mathbb{N}$, define

$$
D_{M} \equiv\left\{\mathbf{x} \in l_{\left(\beta_{i}\right)}^{p}: \beta_{i}^{\frac{1}{p}}\left|x_{i}\right| \leq M \cdot \gamma_{i}^{-\frac{1}{p}}, i \in \mathbb{N}\right\}
$$

then $D_{M}$ is a compact set in $S=l_{\left(\beta_{i}\right)}^{p}$. This is proven through a standard argument as follows: Since $\left\{\gamma_{i}^{-1}\right\}_{i \in \mathbb{N}}$ is assumed to be a positive $l^{1}$ sequence, for any $\epsilon>0$, there exists an $N_{M, \epsilon} \in \mathbb{N}$ and

$$
\sum_{i=N_{M, \epsilon}+1}^{\infty} \beta_{i}\left|x_{i}\right|^{p} \leq \sum_{i=N_{M, \epsilon}+1}^{\infty} M^{p} \cdot \gamma_{i}^{-1} \leq\left(\frac{\epsilon}{3}\right)^{p}, \quad \forall \mathbf{x} \in D_{M} .
$$

Also, for any $\mathbf{x} \in D_{M}$, it holds that (without loss of generality assuming that $0<\gamma_{i}^{-1} \leq$ 1)

$$
\beta_{i}^{\frac{1}{p}}\left|x_{i}\right| \leq M, \quad \forall i=1, \ldots, N_{M, \epsilon}
$$

In view of the above, we can construct an $\epsilon$-net of $D_{M}$ as follows: Take $\epsilon^{\prime} \equiv \frac{\epsilon}{3} \cdot\left(N_{M, \epsilon}\right)^{-\frac{1}{p}}$, and for each $i \leq N_{M, \epsilon}$, set $x_{i, j}$ in order that

$$
\beta_{i}^{\frac{1}{p}} x_{i, j} \equiv-M+\epsilon^{\prime} \cdot j, \quad j=0,1, \ldots,\left[2 M \cdot \epsilon^{\prime-1}\right]+1,
$$


where $[y]$ denotes the greatest integer that is not greater than $y \in \mathbb{R}$. Define finite elements $\mathbf{x}_{j_{1}, j_{2}, \ldots, j_{N_{M, \epsilon}}}$ in $l_{\left(\beta_{i}\right)}^{p}$ as follows:

$$
\begin{gathered}
\mathbf{x}_{j_{1}, j_{2}, \ldots, j_{N_{M, \epsilon}}} \equiv\left(x_{1, j_{1}}, x_{2, j_{2}}, \ldots x_{N_{M, \epsilon}, j_{N_{m}, \epsilon}}, 0,0, \ldots\right) \in l_{\left(\beta_{i}\right)}^{p}, \\
j_{l}=0,1, \ldots,\left[2 M \cdot \epsilon^{\prime-1}\right]+1, \quad l=1, \ldots, N_{M, \epsilon} .
\end{gathered}
$$

On the other hand, by (4.17) and (4.18), for any

$$
\mathbf{x}=\left(x_{1}, x_{2}, \ldots, x_{N_{M, \epsilon}}, x_{N_{M, \epsilon}+1}, \ldots\right) \in D_{M}
$$

there exists a vector $\left(x_{1, j_{1}}, x_{2, j_{2}}, \ldots x_{N_{M, \epsilon}, j_{N_{m, \epsilon}}}\right) \in \mathbb{R}^{N_{M, \epsilon}}$ such that

$$
\left|\beta_{i}^{\frac{1}{p}} x_{i}-\beta_{i}^{\frac{1}{p}} x_{i, j}\right|<\epsilon^{\prime}, \quad \forall i=1, \ldots, N_{M, \epsilon}, \quad x_{i} \text { as in (4.17), } x_{i, j} \text { as in (4.18). }
$$

By combining (4.16) with (4.21), for any $\mathbf{x} \in D_{M}$ with the expression (4.20), there

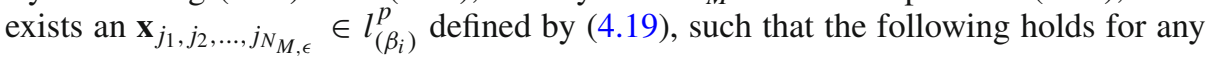
$\epsilon>0$ :

$$
\begin{aligned}
\left\|\mathbf{x}-\mathbf{x}_{j_{1}, j_{2}, \ldots, j_{N_{M}, \epsilon}}\right\|_{l_{\left(\beta_{i}\right)}^{p}}^{p} & <\sum_{i=1}^{N_{M, \epsilon}} \beta_{i}\left|x_{i}-x_{i, j}\right|^{p}+\sum_{i=N_{M, \epsilon}+1}^{\infty} \beta_{i}\left|x_{i}\right|^{p} \\
& \leq \sum_{i=1}^{N_{M, \epsilon}}\left(\epsilon^{\prime}\right)^{p}+\left(\frac{\epsilon}{3}\right)^{p} \leq\left(\frac{\epsilon}{3}\right)^{p}+\left(\frac{\epsilon}{3}\right)^{p}<\epsilon^{p} .
\end{aligned}
$$

(4.22) shows that for any $\epsilon>0$ there exists a finite (cf. (4.19)) open covering of $D_{M}$ such that

$$
D_{M} \subset \bigcup_{j_{1}, \ldots, j_{N_{M, \epsilon}}}\left\{\mathbf{x}^{\prime} \in l_{\left(\beta_{i}\right)}^{p}:\left\|\mathbf{x}^{\prime}-\mathbf{x}_{j_{1}, \ldots, j_{N_{m}, \epsilon}}\right\|_{l_{\left(\beta_{i}\right)}^{p}}<\epsilon\right\} .
$$

Hence, for the subset $D_{M}$ of $S=l_{\left(\beta_{i}\right)}^{p}$, there exists an $\epsilon$ net and it is totally bounded. Since, obviously, $D_{M}$ is a closed set and since $l_{\left(\beta_{i}\right)}^{p}$ is a complete metric space (cf. Remark 1-iii)), by Fréchet's compactness criterion for complete metric spaces, we see that for each $M \in \mathbb{N}, D_{M}$ is a compact subset of $l_{\left(\beta_{i}\right)}^{p}$.

Next, we proceed to define $\tilde{\mathcal{D}}\left(\mathcal{E}_{(\alpha)}\right)$ for which (4.13) and (4.14) hold. Let $\eta(\cdot) \in$ $C_{0}^{\infty}(\mathbb{R})$ be a function such that $0 \leq \eta(x) \leq 1,\left|\frac{d}{d x} \eta(x)\right| \leq 1, \forall x \in \mathbb{R}$ and

$$
\eta(x)= \begin{cases}1, & |x| \leq 1 \\ 0, & |x| \geq 3\end{cases}
$$

For each $M \in \mathbb{N}$ and $i \in \mathbb{N}$, let

$$
\eta_{M, i}(x) \equiv \eta\left(M^{-1} \cdot \gamma_{i}^{\frac{1}{p}} \beta_{i}^{\frac{1}{p}} \cdot x\right), \quad x \in \mathbb{R},
$$

then, by (4.15), (4.24) and (4.25), we see that

$$
\operatorname{supp}\left[\prod_{i \geq 1} \eta_{M, i}\right] \subset D_{3 M}, \quad M \in \mathbb{N} .
$$


For each $f \in C_{0}^{\infty}\left(\mathbb{R}^{n} \rightarrow \mathbb{R}\right), n \in \mathbb{N}$, define

$f_{M}\left(x_{1}, \ldots, x_{n}, x_{n+1}, \ldots\right) \equiv f\left(x_{1}, \ldots, x_{n}\right) \cdot \prod_{i \geq 1} \eta_{M, i}\left(x_{i}\right), \quad \mathbf{x}=\left(x_{1}, x_{2}, \ldots\right) \in \mathbb{R}^{\mathbb{N}}$,

and then define a subspace $\tilde{\mathcal{D}}\left(\mathcal{E}_{(\alpha)}\right) \subset L^{2}(S ; \mu)$, that is the linear span of the family of $f_{M}^{\prime} s, M \in \mathbb{N}, f \in C_{0}^{\infty}\left(\mathbb{R}^{n} \rightarrow \mathbb{R}\right), n \in \mathbb{N}$, defined by (4.27), i.e., the space of finite linear combinations of $f_{M}^{\prime} s$ defined by (4.27):

$$
\tilde{\mathcal{D}}\left(\mathcal{E}_{(\alpha)}\right) \equiv \text { the linear span of }\left\{f_{M}, M \in \mathbb{N}: f \in C_{0}^{\infty}\left(\mathbb{R}^{n} \rightarrow \mathbb{R}\right), n \in \mathbb{N}\right\}
$$

Note that $\mathcal{F} C_{0}^{\infty}$ defined by (2.5) is expressed in a similar way as (4.27):

$$
\mathcal{F} C_{0}^{\infty}=\left\{f\left(x_{1}, \ldots, x_{n}\right) \cdot \prod_{i \geq 1} I_{\mathbb{R}}\left(x_{i}\right): f \in C_{0}^{\infty}\left(\mathbb{R}^{n} \rightarrow \mathbb{R}\right), n \in \mathbb{N}\right\},
$$

where $I_{A}(x), A \in \mathcal{B}(\mathbb{R})$, denotes the indicator function. Then, for any $f_{M}$, defined by (4.27), supp $\left[f_{M}\right] \subset D_{M^{\prime}}$ (cf. (4.26)) holds for all $M^{\prime} \geq 3 M$. Thus, if we see that

$$
\tilde{\mathcal{D}}\left(\mathcal{E}_{(\alpha)}\right) \subset \mathcal{D}\left(\mathcal{E}_{(\alpha)}\right),
$$

and for any $u \equiv f \cdot \prod_{i>1} I_{\mathbb{R}}\left(x_{i}\right) \in \mathcal{F} C_{0}^{\infty} \subset \mathcal{D}\left(\mathcal{E}_{(\alpha)}\right)$ (cf. (4.29)), there exists a sequence $\left\{w_{m}\right\}_{m \in \mathbb{N}}, w_{m} \in \tilde{\mathcal{D}}\left(\mathcal{E}_{(\alpha)}\right), m \in \mathbb{N}$, such that

$$
\lim _{m \rightarrow \infty} w_{m}=u \equiv f \cdot \prod_{i \geq 1} I_{\mathbb{R}}\left(x_{i}\right), \quad \text { in } \mathcal{D}\left(\mathcal{E}_{(\alpha)}\right),
$$

then (4.13) and (4.14) are verified.

Let us show that (4.30) and (4.31) hold. For each $M \in \mathbb{N}$. Denote (cf. (4.15) and (4.26))

$$
a_{M, i} \equiv M \gamma_{i}^{-\frac{1}{p}} \beta_{i}^{-\frac{1}{p}}, \quad b_{M, i} \equiv 3 M \gamma_{i}^{-\frac{1}{p}} \beta_{i}^{-\frac{1}{p}}, \quad i \in \mathbb{N} .
$$

Below, we use simplified notations for the indicator functions such that, e.g., $I_{\left[-a_{M, i}, a_{M, i}\right]}(y)=I_{|y| \leq a_{M, i}}$ and $I_{\left(a_{M, i}, b_{M, i}\right]}(y)=1_{a_{M, i}<y \leq b_{M, i}}$ and so on. From the definition of $\eta$ (cf. (4.24) and (4.25)), since $0 \leq \eta_{M, i}(x) \leq 1(\forall x \in \mathbb{R})$, and by the mean value theorem, since for any $x<y$, there exists a $\theta \in(0,1)$ such that $\eta_{M, i}(x)-\eta_{M, i}(y)=\eta_{M_{i}}^{\prime}(x+\theta \cdot(y-x)) \cdot(y-x)$, we have the following bound for $0<\alpha \leq 1$ :

$$
\begin{aligned}
\left|\eta_{M, i}(x)-\eta_{M, i}(y)\right|^{2} & =\left|\eta_{M, i}(x)-\eta_{M, i}(y)\right|^{\alpha+1}\left|\eta_{M, i}(x)-\eta_{M, i}(y)\right|^{1-\alpha} \\
& \leq 2^{1-\alpha}\left|\eta_{M, i}(x)-\eta_{M, i}(y)\right|^{\alpha+1} \\
& \leq 2^{1-\alpha}\left|\eta_{M_{i}}^{\prime}(x+\theta \cdot(y-x))\right|^{\alpha+1} \cdot|x-y|^{\alpha+1} .
\end{aligned}
$$

By making use of this bound and noting that $\eta_{M, i}(x)=\eta_{M, i}(y)=1$ for $|x|,|y| \leq a_{M, i}$, from the definition (4.25) for $\eta_{M_{i}}(x)$, we then have the following evaluation:

$$
\frac{\left(\eta_{M, i}(y)-\eta_{M, i}(x)\right)^{2}}{|y-x|^{\alpha+1}} I_{|y| \leq a_{M, i}} I_{x \neq y}
$$




$$
\begin{aligned}
= & \frac{\left(\eta_{M, i}(y)-\eta_{M, i}(x)\right)^{2}}{|y-x|^{\alpha+1}} \cdot\left(I_{|y| \leq a_{M, i}} I_{|x| \leq a_{M, i}}+I_{|y| \leq a_{M, i}} I_{-b_{M, i} \leq x<-a_{M, i}}\right. \\
& \left.+I_{|y| \leq a_{M, i}} I_{a_{M, i}<x \leq b_{M, i}}+I_{|y| \leq a_{M, i}} I_{|x|>b_{M, i}}\right) I_{x \neq y} \\
\leq & 2^{1-\alpha}\left(\sup _{t \in \mathbb{R}}\left|\frac{d}{d t} \eta_{M, i}(t)\right|^{\alpha+1}\right) \cdot\left(I_{|y| \leq a_{M, i}} I_{-b_{M, i} \leq x<-a_{M, i}}\right. \\
& \left.+I_{|y| \leq a_{M, i}} I_{a_{M, i}<x \leq b_{M, i}}+I_{|y| \leq a_{M, i}} I_{|x|>b_{M, i}}\right) I_{x \neq y} \\
\leq & 2^{1-\alpha}\left(\sup _{t \in \mathbb{R}}\left|\frac{d}{d t} \eta_{M, i}(t)\right|^{\alpha+1}\right) \cdot\left(I_{x<-a_{M, i}}+I_{a_{M, i}<x}+I_{|x|>b_{M, i}}\right) I_{x \neq y} \\
\leq & 2^{1-\alpha} 3\left(M^{-1} \gamma_{i}^{\frac{1}{p}} \beta_{i}^{\frac{1}{p}}\right)^{\alpha+1} I_{|x|>a_{M, i}} I_{x \neq y} .
\end{aligned}
$$

Similarly,

$$
\begin{aligned}
& \frac{\left(\eta_{M, i}(y)-\eta_{M, i}(x)\right)^{2}}{|y-x|^{\alpha+1}}\left(I_{-b_{M, i} \leq y<-a_{M, i}}+I_{a_{M, i}<y \leq b_{M, i}}\right) I_{x \neq y} \\
& \leq 2 \cdot 2^{1-\alpha}\left(\sup _{t \in \mathbb{R}}\left|\frac{d}{d t} \eta_{M, i}(t)\right|^{\alpha+1}\right) \cdot I_{|y|>a_{M, i}} I_{x \neq y} \\
& \leq 2^{2-\alpha}\left(M^{-1} \gamma_{i}^{\frac{1}{p}} \beta_{i}^{\frac{1}{p}}\right)^{\alpha+1} I_{|y|>a_{M, i}} I_{x \neq y},
\end{aligned}
$$

and

$$
\begin{aligned}
& \frac{\left(\eta_{M, i}(y)-\eta_{M, i}(x)\right)^{2}}{|y-x|^{\alpha+1}} I_{|y|>b_{M, i}} I_{x \neq y} \\
& \leq 2^{1-\alpha}\left(\sup _{t \in \mathbb{R}}\left|\frac{d}{d t} \eta_{M, i}(t)\right|^{\alpha+1}\right) \cdot I_{|y|>b_{M, i}} I_{x \neq y} \\
& \leq 2^{1-\alpha}\left(M^{-1} \gamma_{i}^{\frac{1}{p}} \beta_{i}^{\frac{1}{p}}\right)^{\alpha+1} I_{|y|>a_{M, i}} I_{x \neq y} .
\end{aligned}
$$

Next, for each $f \in C_{0}^{\infty}\left(\mathbb{R}^{n} \rightarrow \mathbb{R}\right), n \in \mathbb{N}$, define $f_{M, k} \in \mathcal{F} C_{0}^{\infty}, k \geq n$, (cf. (4.27) and (4.29)) as follows:

$$
f_{M, k}\left(x_{1}, \ldots, x_{n}, x_{n+1}, \ldots\right) \equiv f\left(x_{1}, \ldots, x_{n}\right) \cdot \prod_{i=1}^{k} \eta_{M, i}\left(x_{i}\right) \prod_{j \geq k+1} I_{\mathbb{R}}\left(x_{j}\right), \quad k \geq n .
$$

In the sequel for $f_{M, k}$ defined through $f \in C_{0}^{\infty}\left(\mathbb{R}^{n} \rightarrow \mathbb{R}\right)$, we assume, without mentioning it explicitly, that $M$ is taken sufficiently large in order that the following holds:

$$
\operatorname{supp}[f] \subset\left[-M \gamma_{1}^{-\frac{1}{p}} \beta_{1}^{-\frac{1}{p}},+M \gamma_{1}^{-\frac{1}{p}} \beta_{1}^{-\frac{1}{p}}\right] \times \cdots \times\left[-M \gamma_{n}^{-\frac{1}{p}} \beta_{n}^{-\frac{1}{p}},+M \gamma_{n}^{-\frac{1}{p}} \beta_{n}^{-\frac{1}{p}}\right],
$$

i.e., by (4.25), we assume that $M$ is taken sufficiently large in order that $\prod_{i=1}^{n} \eta_{M, i}\left(x_{i}\right)=1$ holds for $\left(x_{1}, \ldots, x_{n}\right) \in \operatorname{supp}[f]$. Thus, to define $f_{M, k}$ by (4.36), assume that $M<\infty$ is taken sufficiently large and the following holds:

$$
f\left(x_{1}, \ldots, x_{n}\right) \cdot \prod_{i=1}^{n} \eta_{M, i}\left(x_{i}\right)=f\left(x_{1}, \ldots, x_{n}\right), \quad \forall\left(x_{1}, \ldots, x_{n}\right) \in \mathbb{R}^{n} .
$$


Let (cf. (4.29), and (4.36))

$$
u \equiv f \cdot \prod_{i \geq n+1} I_{\mathbb{R}}\left(x_{i}\right) \in \mathcal{F} C_{0}^{\infty},
$$

then, by this and (4.36) with the above equality, which holds for sufficiently large $M<$ $\infty$, for $n \leq k$ we have

$$
\begin{aligned}
& \left(f_{M, k}\left(x_{1}, \ldots, x_{i-1}, y_{i}, x_{i+1}, \ldots, x_{n}, \ldots\right)-f_{M, k}\left(x_{1}, \ldots, x_{i-1}, y_{i}^{\prime}, x_{i+1}, \ldots, x_{n}, \ldots\right)\right)^{2} \\
& \leq\left(u\left(x_{1}, \ldots, x_{i-1}, y_{i}, x_{i+1}, \ldots, x_{n}, \ldots\right)-u\left(x_{1}, \ldots, x_{i-1}, y_{i}^{\prime}, x_{i+1}, \ldots, x_{n}, \ldots\right)\right)^{2} \\
& \quad \forall \mathbf{x} \in \mathbb{R}^{\mathbb{N}}, \quad \forall y_{i}, y_{i}^{\prime} \in \mathbb{R}, \quad 1 \leq i \leq n
\end{aligned}
$$

By making use of (4.33), (4.34) and (4.35), we have the following evaluation for the quadratic form $\mathcal{E}_{(\alpha)} \equiv \sum_{i \in \mathbb{N}} \mathcal{E}_{(\alpha)}^{(i)}$, (cf. (2.10)), defined through $\mathcal{E}_{(\alpha)}^{(i)}$ given by (2.13) in Remark 2, that is equivalent to the formula (2.9). For $k \geq n$, let $f_{M, k}$, be the function defined by (4.36) satisfying (4.37). Then for $1 \leq i \leq n$, since $\Phi_{\alpha}\left(f_{M, k}, f_{M, k} ; y_{i}, y_{i}^{\prime}, \mathbf{x} \backslash x_{i}\right) \leq \Phi_{\alpha}\left(u, u ; y_{i}, y_{i}^{\prime}, \mathbf{x} \backslash x_{i}\right)$, for all $y, y^{\prime}, \mathbf{x}$, it holds that, for $1 \leq i \leq n$,

$$
\mathcal{E}_{(\alpha)}^{(i)}\left(f_{M, k}, f_{M, k}\right) \leq \mathcal{E}_{(\alpha)}^{(i)}(u, u)
$$

also for $n+1 \leq i \leq k$, by (4.33), (4.34) and (4.35), with $C_{\alpha} \equiv 6 \cdot 2^{1-\alpha}$,

$$
\begin{aligned}
\mathcal{E}_{(\alpha)}^{(i)} & \left(f_{M, k}, f_{M, k}\right) \\
= & \int_{S}\left(f\left(x_{1}, \ldots, x_{n}\right)\right)^{2} \cdot \prod_{j \geq n+1, j \neq i}^{k}\left(\eta_{M, j}\left(x_{j}\right)\right)^{2} \prod_{l \geq k+1}\left(I_{\mathbb{R}}\left(x_{l}\right)\right)^{2} \\
& \times\left\{\int_{\mathbb{R}^{2}} I_{y_{i} \neq y_{i}^{\prime}} \frac{1}{\left|y_{i}-y_{i}^{\prime}\right|^{\alpha+1}}\left(\eta_{M, i}\left(y_{i}\right)-\eta_{M, i}\left(y_{i}^{\prime}\right)\right)^{2} \mu\left(d y_{i} \mid \sigma_{i^{c}}\right) \mu\left(d y_{i}^{\prime} \mid \sigma_{i^{c}}\right)\right\} \mu(d \mathbf{x}) \\
\leq & C_{\alpha}\left(M^{-1} \gamma_{i}^{\frac{1}{p}} \beta_{i}^{\frac{1}{p}}\right)^{\alpha+1} \int_{S}\left(f\left(x_{1}, \ldots, x_{n}\right)\right)^{2} \prod_{j \neq i} I_{\mathbb{R}}\left(x_{j}\right)\left\{\int_{\mathbb{R}} I_{\left|y_{i}^{\prime}\right|>a_{M, i}} \mu\left(d y_{i}^{\prime} \mid \sigma_{i^{c}}\right)\right\} \mu(d \mathbf{x}) \\
\leq & C_{\alpha}\left(M^{-1} \gamma_{i}^{\frac{1}{p}} \beta_{i}^{\frac{1}{p}}\right)^{\alpha+1}\|f\|_{L^{\infty}}^{2} \int_{S}\left\{\int_{\mathbb{R}} I_{\left|y_{i}^{\prime}\right|>a_{M, i}} \mu\left(d y_{i}^{\prime} \mid \sigma_{i}^{c}\right)\right\} \mu(d \mathbf{x}) \\
\leq & C_{\alpha}\left(M^{-1} \gamma_{i}^{\frac{1}{p}} \beta_{i}^{\frac{1}{p}}\right)^{\alpha+1}\|f\|_{L^{\infty}}^{2} \mu\left(\left|x_{i}\right|>a_{M, i}\right),
\end{aligned}
$$

and for $k+1 \leq i$, since $\Phi_{\alpha}\left(f_{M, k}, f_{M, k} ; y_{i}, y_{i}^{\prime}, \mathbf{x} \backslash x_{i}\right)=0$, for all $y, y^{\prime}, \mathbf{x}$, by (4.36), for $k+1 \leq i$,

$$
\mathcal{E}_{(\alpha)}^{(i)}\left(f_{M, k}, f_{M, k}\right)=0 .
$$

By combining (4.38), (4.39) and (4.40), from (4.32), the definition of $a_{M, i}$, for $f_{M, k}$ defined through $f \in C_{0}^{\infty}\left(\mathbb{R}^{n} \rightarrow \mathbb{R}\right), n \in \mathbb{N}$ we have

$$
\begin{aligned}
& \mathcal{E}_{(\alpha)}^{(i)}\left(f_{M, k}, f_{M, k}\right) \leq \mathcal{E}_{(\alpha)}^{(i)}(u, u)+C_{\alpha}\left(M^{-1} \gamma_{i}^{\frac{1}{p}} \beta_{i}^{\frac{1}{p}}\right)^{\alpha+1}\|f\|_{L^{\infty}}^{2} \cdot \mu\left(\left|X_{i}\right|>M \gamma_{i}^{-\frac{1}{p}} \beta_{i}^{-\frac{1}{p}}\right), \\
& \quad \text { for any } k \geq n .
\end{aligned}
$$

By (4.41), for $f_{M, k}$ defined through $f \in C_{0}^{\infty}\left(\mathbb{R}^{n} \rightarrow \mathbb{R}\right), n \in \mathbb{N}$, in order to make the discussions simpler by taking $M$ satisfying both (4.37) and $M \geq M_{0}$ (cf. (4.3)), 
since $\mu\left(\left|X_{i}\right|>M \gamma_{i}^{-\frac{1}{p}} \beta_{i}^{-\frac{1}{p}}\right)$ is a decreasing function of $M>0$, we see that if the condition (4.3) of Theorem 2-i) is satisfied, then the following holds: There exists a constant $C_{\alpha}<\infty$ that does not depend on $M \geq M_{0}$ and

$$
\sup _{k \geq 1} \mathcal{E}_{(\alpha)}\left(f_{M, k}, f_{M, k}\right)=\sup _{k \geq 1} \sum_{i \in \mathbb{N}} \mathcal{E}_{(\alpha)}^{(i)}\left(f_{M, k}, f_{M, k}\right) \leq \mathcal{E}_{(\alpha)}(u, u)+C_{\alpha} M^{-(\alpha+1)}\|f\|_{L^{\infty}}^{2},
$$

for any sufficiently large $M$ by which satisfies (4.37) holds with $M \geq M_{0}$.

Since, by the definitions (4.27) and (4.36), $\lim _{k \rightarrow \infty} f_{M, k}=f_{M}, \mu-$ a.e. , and by Lebesgue's bounded convergence theorem $\lim _{k \rightarrow \infty}\left\|f_{M, k}-f_{M}\right\|_{L^{2}(S ; \mu)}=0$, (4.42) shows that the sequence $\left\{f_{M, k}\right\}_{k \in \mathbb{N}}, f_{M, k} \in \mathcal{F} C_{0}^{\infty} \subset \mathcal{D}\left(\mathcal{E}_{(\alpha)}\right)$ satisfies the condition for (4.10) of Lemma 1. Hence, we conclude that for $f_{M}$ defined by (4.27) through $f \in C_{0}^{\infty}\left(\mathbb{R}^{n} \rightarrow \mathbb{R}\right), n \in \mathbb{N}$, and the subspace $\tilde{\mathcal{D}}\left(\mathcal{E}_{(\alpha)}\right) \subset L^{2}(S ; \mu)$ defined by (4.28) the following is true (cf. (4.10)):

$$
f_{M} \in \mathcal{D}\left(\mathcal{E}_{(\alpha)}\right), \quad \text { and } \quad \tilde{\mathcal{D}}\left(\mathcal{E}_{(\alpha)}\right) \subset \mathcal{D}\left(\mathcal{E}_{(\alpha)}\right),
$$

also by (4.42) (cf. (4.10)),

$$
\mathcal{E}_{(\alpha)}\left(f_{M}, f_{M}\right) \leq \liminf _{k \rightarrow \infty} \mathcal{E}_{(\alpha)}\left(f_{M, k}, f_{M, k}\right) \leq \mathcal{E}_{(\alpha)}(u, u)+C_{\alpha} M^{-(\alpha+1)}\|f\|_{L^{\infty}}^{2},
$$

for any sufficiently large $M$ by which satisfies (4.37) holds with $M \geq M_{0}$. (4.43) shows (4.30).

Finally, we apply the same arguments to the case of the sequence $\left\{f_{M}\right\}_{M \in \mathbb{N}}, f_{M} \in$ $\mathcal{D}\left(\mathcal{E}_{(\alpha)}\right)$. From (4.44) we have

$$
\sup _{M \in \mathbb{N}} \mathcal{E}_{(\alpha)}\left(f_{M}, f_{M}\right)<\infty
$$

Moreover, by (4.15), (4.25) with (4.24) (cf. (4.26)), since

$$
\begin{gathered}
A_{M} \equiv\left\{\mathbf{x}: \prod_{i \geq 1} I_{\mathbb{R}}\left(x_{i}\right)-\prod_{i \geq 1} \eta_{M, i}\left(x_{i}\right) \neq 0\right\}=\left\{\mathbf{x}: \exists i \in \mathbb{N}, \eta_{M, i}\left(x_{i}\right) \neq 1\right\} \\
\subset\left\{\mathbf{x}: \exists i \in \mathbb{N},\left|x_{i}\right|>M \gamma_{i}^{-\frac{1}{p}} \beta_{i}^{-\frac{1}{p}}\right\}=\bigcup_{i \geq 1}\left\{\mathbf{x}:\left|x_{i}\right|>M \gamma_{i}^{-\frac{1}{p}} \beta_{i}^{-\frac{1}{p}}\right\},
\end{gathered}
$$

for $u \equiv f \cdot \prod_{i \geq 1} I_{\mathbb{R}}\left(x_{i}\right) \in \mathcal{F} C_{0}^{\infty}$ and $f_{M}$ defined by (4.27), we see that

$$
\begin{aligned}
& \left\|u-f_{M}\right\|_{L^{2}(S ; \mu)} \leq\|f\|_{L^{\infty}} \int_{S} I_{A_{M}}(\mathbf{x}) \mu(d \mathbf{x}) \\
& =\|f\|_{L^{\infty}} \mu\left(\bigcup_{i \geq 1}\left\{\mathbf{x}:\left|x_{i}\right|>M \gamma_{i}^{-\frac{1}{p}} \beta_{i}^{-\frac{1}{p}}\right\}\right)=\|f\|_{L^{\infty}}\left(1-\mu\left(D_{M}\right)\right),
\end{aligned}
$$

But, under the condition (4.4), it holds that

$$
\begin{aligned}
& \limsup _{M \rightarrow \infty}\left(1-\mu\left(D_{M}\right)\right)=1-\liminf _{M \rightarrow \infty} \mu\left(D_{M}\right) \leq 1-\mu\left(\liminf _{M \rightarrow \infty} D_{M}\right) \\
& \quad=1-\mu\left(\cup_{M \in \mathbb{N}} D_{M}\right)=0 .
\end{aligned}
$$


Thus, $\lim _{M \rightarrow \infty}\left\|f_{M}-u\right\|_{L^{2}(S ; \mu)}=0$, and hence (4.45) shows that the sequence $\left\{f_{M}\right\}_{M \in \mathbb{N}}, f_{M} \in \mathcal{D}\left(\mathcal{E}_{(\alpha)}\right)$ satisfies the condition for (4.10) of Lemma 1. Then, by (4.11), the second assertion of Lemma 1 , shows that there exists a subsequence $\left\{f_{M_{l}}\right\}_{l \in \mathbb{N}}$ of $\left\{f_{M}\right\}_{M \in \mathbb{N}}$ such that the Cesàro mean

$$
w_{m} \equiv \frac{1}{m} \sum_{l=1}^{m}\left(f_{M_{l}}\right) \rightarrow u \equiv f \cdot \prod_{i \geq 1} I_{\mathbb{R}}\left(x_{i}\right) \quad \text { in } \mathcal{D}\left(\mathcal{E}_{(\alpha)}\right) \text { as } n \rightarrow \infty .
$$

This shows (4.31). Since, as we have mentioned before, from the definition (4.28) and by (4.43), $w_{m} \in \tilde{\mathcal{D}}\left(\mathcal{E}_{(\alpha)}\right) \subset \mathcal{D}\left(\mathcal{E}_{(\alpha)}\right)$ holds, (4.43) and (4.46) verify (4.13) and (4.14), respectively. This complete the proof of Theorem 2-i), for the case $S=l_{\left(\beta_{i}\right)}^{p}, 1 \leq p<\infty$.

Proof of (4.13) and (4.14) for $S=l_{\left(\beta_{i}\right)}^{\infty}$. The same arguments, through which (4.13) and (4.14) are verified for the case where $S=l_{\left(\beta_{i}\right)}^{p}, 1 \leq p<\infty$, can be applied to the case where $S=l_{\left(\beta_{i}\right)}^{\infty}$ as defined by (2.2). Namely, for $S=l_{\left(\beta_{i}\right)}^{\infty}$, a definition of the compact set $D_{M}$ corresponding to (4.15) is

$$
D_{M} \equiv\left\{\mathbf{x} \in l_{\left(\beta_{i}\right)}^{\infty}: \beta_{i}\left|x_{i}\right| \leq M \cdot \gamma_{i}^{-1}, i \in \mathbb{N}\right\}, \quad M \in \mathbb{N},
$$

where $\left\{\gamma_{i}\right\}_{i \in \mathbb{N}}$ is any sequence such that $0<\gamma_{1} \leq \gamma_{2} \leq \cdots \rightarrow \infty$, and the evaluation corresponding to (4.41) is

$$
\begin{aligned}
& \mathcal{E}_{(\alpha)}^{(i)}\left(f_{M, k}, f_{M, k}\right) \leq \mathcal{E}_{(\alpha)}^{(i)}(u, u)+C_{\alpha}\left(M^{-1} \gamma_{i} \beta_{i}\right)^{\alpha+1}\|f\|_{L^{\infty}} \cdot \mu\left(\left|X_{i}\right|>M \gamma_{i}^{-1} \beta_{i}^{-1}\right), \\
& \quad \text { for any } k \geq n .
\end{aligned}
$$

Passing through the same arguments by which (4.46) is derived (cf. (4.45)), under the conditions (4.5) with (4.6), we can prove (4.13) and (4.14) for $S=l_{\left(\beta_{i}\right)}^{\infty}$, and Theorem 2ii) follows.

Proof of (4.13) and (4.14) for $S=\mathbb{R}^{\mathbb{N}}$. Similar to the above, the same arguments, through which (4.13) and (4.14) are verified for the case where $S=l_{\left(\beta_{i}\right)}^{p}, 1 \leq p<\infty$, can be applied to the case where $S=\mathbb{R}^{\mathbb{N}}$ defined by (2.3). Namely, for $S=\mathbb{R}^{\mathbb{N}}$, a definition of the compact set $D_{M}$ corresponding to (4.15) is

$$
D_{M} \equiv\left\{\mathbf{x} \in \mathbb{R}^{\mathbb{N}}:\left|x_{i}\right| \leq M \cdot \gamma_{i}, \quad i \in \mathbb{N}\right\}, \quad M \in \mathbb{N},
$$

where $\left\{\gamma_{i}\right\}_{i \in \mathbb{N}}$ is any sequence such that $\gamma_{i}>0, \forall i \in \mathbb{N}$, and the evaluation corresponding to (4.41) is

$$
\mathcal{E}_{(\alpha)}^{(i)}\left(f_{M_{k}}, f_{M_{k}}\right) \leq \mathcal{E}_{(\alpha)}^{(i)}(u, u)+C_{\alpha}\left(M^{-1} \gamma_{i}^{-1}\right)^{\alpha+1}\|f\|_{L^{\infty}}^{2} \cdot \mu\left(\left|X_{i}\right|>M \gamma_{i}\right), k \geq n .
$$

For the probability distribution of the real valued random variable $X_{i}$, since

$$
\mu\left(\left|X_{i}\right|>M \gamma_{i}\right) \leq 1, \quad \forall i \in \mathbb{N}, \quad \forall M \geq 0, \quad \forall \gamma_{i} \geq 0,
$$

by taking $\gamma_{i} \equiv i, i \in \mathbb{N}$, in (4.50), then from (4.50) and (4.51) we see that, for any $f \in C_{0}^{\infty}\left(\mathbb{R}^{n} \rightarrow \mathbb{R}\right), n \in \mathbb{N}$,

$$
\mathcal{E}_{(\alpha)}\left(f_{M}, f_{M}\right) \leq \mathcal{E}_{(\alpha)}(u, u)+C_{\alpha} M^{-(\alpha+1)} \sum_{i=1}^{\infty} i^{-(\alpha+1)}<\infty, \quad \forall M \in \mathbb{N} .
$$


Thus, by passing through the same arguments by which (4.46) is derived (cf. (4.45)), we prove (4.13) and (4.14) for $S=\mathbb{R}^{\mathbb{N}}$, and Theorem 2-iii) follows.

Next, let us prove iv). The point iv) is assured when we show that $1 \in \mathcal{D}\left(\mathcal{E}_{(\alpha)}\right)$. The proof is common to all the state spaces we are considering, namely $l_{\left(\beta_{i}\right)}^{p}, l_{\left(\beta_{i}\right)}^{\infty}, \mathbb{R}^{\mathbb{N}}$.

Take $\eta \in C_{0}^{\infty}(\mathbb{R} \rightarrow \mathbb{R})$ as (4.24), namely let $\eta$ be such that $\eta(x) \geq 0,\left|\frac{d}{d x} \eta(x)\right| \leq 1$ for $x \in \mathbb{R}$, and $\eta(x)=1$ for $|x|<1 ; \eta(x)=0$ for $|x|>3$, and define $u_{M}\left(x_{1}, x_{2}, \ldots\right) \equiv$ $\eta\left(x_{1} \cdot M^{-1}\right) \prod_{i>2} I_{\mathbb{R}}\left(x_{i}\right) \in \mathcal{F} C_{0}^{\infty} \subset \mathcal{D}\left(\mathcal{E}_{(\alpha)}\right)$ for each $M \in \mathbb{N}$. Then, for $0<\alpha \leq 1$, by (2.8) (cf. (4.33)-(4.35)), for any sufficiently large $M$, since $0 \leq \Phi_{\alpha}\left(u_{M} \cdot u_{M} ; y_{1}, y_{1}^{\prime}, \mathbf{x} \backslash\right.$ $\left.x_{1}\right) \leq 1$, for all $y_{1}, y_{1}^{\prime} \in \mathbb{R}$ and all $\mathbf{x} \in S$, and for $i \neq 1, \Phi_{\alpha}\left(u_{M} \cdot u_{M} ; y_{i}, y_{i}^{\prime}, \mathbf{x} \backslash x_{i}\right)=0$ for all $y_{i}, y_{i}^{\prime} \in \mathbb{R}$ and all $\mathbf{x} \in S$, we see that (using (2.9) and (2.10) and estimates similar to those in (4.33)-(4.35)) $\sup _{M \in \mathbb{N}} \mathcal{E}_{(\alpha)}\left(u_{M}, u_{M}\right)<\infty$. Since, $\lim _{M \rightarrow \infty} u_{M}(\mathbf{x})=1=$ $\prod_{i>1} I_{\mathbb{R}}\left(x_{i}\right)$ pointwise, and also in $L^{2}(S ; \mu)$, it follows from the first part of Lemma 1 that we have $1 \in \mathcal{D}\left(\mathcal{E}_{(\alpha)}\right)$.

This completes the proof of Theorem 2 .

In the case where $1<\alpha<2$, for the quasi-regularity of the Dirichlet form $\left(\mathcal{E}_{(\alpha)}, \mathcal{D}\left(\mathcal{E}_{(\alpha)}\right)\right)$ considered in Theorem 1, the following Theorem 3 holds:

Theorem 3. Let $1<\alpha<2$. Suppose that the assumption (2.11) with (2.12) hold. Let $\left(\mathcal{E}_{(\alpha)}, \mathcal{D}\left(\mathcal{E}_{(\alpha)}\right)\right)$ be the closed Markovian symmetric form defined at the beginning of this section through Theorem 1. Then the following statements hold:

(i) In the case where $S=l_{\left(\beta_{i}\right)}^{p}$, for some $1 \leq p<\infty$, as defined by (2.1), suppose that (4.4) holds and that there exists a positive $l^{1}$ sequence $\left\{\gamma_{i}^{-1}\right\}_{i \in \mathbb{N}}$ and an $M_{0} \in(0, \infty)$, such that

$$
\sum_{i=1}^{\infty}\left(\beta_{i}^{\frac{1}{p}} \gamma_{i}^{\frac{1}{p}}\right)^{\alpha+1} \cdot \mu\left(\beta_{i}^{\frac{1}{p}}\left|X_{i}\right|>M_{0} \cdot \gamma_{i}^{-\frac{1}{p}}\right)<\infty,
$$

holds. Also suppose that

$$
\sup _{M \geq M_{0}} M^{-\alpha} \sum_{i=1}^{\infty} L_{M, i} \cdot\left(\beta_{i}^{\frac{1}{p}} \gamma_{i}^{\frac{1}{p}}\right)^{\alpha} \cdot \mu\left(\beta_{i}^{\frac{1}{p}}\left|X_{i}\right|>M \cdot \gamma_{i}^{-\frac{1}{p}}\right)<\infty,
$$

holds, where for each $M<\infty$ and $i \in \mathbb{N}, L_{M, i}$ is the bound, assumed in assumption (2.12), for the conditional probability density $\rho$ corresponding to the compact set

$$
K(M, i) \equiv\left[-3 M \cdot \beta_{i}^{-\frac{1}{p}} \gamma_{i}^{-\frac{1}{p}}, 3 M \cdot \beta_{i}^{-\frac{1}{p}} \gamma_{i}^{-\frac{1}{p}}\right] \subset \mathbb{R} .
$$

Then $\left(\mathcal{E}_{(\alpha)}, \mathcal{D}\left(\mathcal{E}_{(\alpha)}\right)\right)$ is a strictly quasi-regular Dirichlet form.

(ii) In the case where $S=l_{\left(\beta_{i}\right)}^{\infty}$ as defined by (2.2), suppose that (4.6) holds, and that there exists a sequence $\left\{\gamma_{i}\right\}_{i \in \mathbb{N}}$ such that $0<\gamma_{1} \leq \gamma_{2} \leq \cdots \rightarrow \infty$ and an $0<M_{0}<\infty$, and

$$
\sum_{i=1}^{\infty}\left(\beta_{i} \gamma_{i}\right)^{\alpha+1} \cdot \mu\left(\beta_{i}\left|X_{i}\right|>M_{0} \cdot \gamma_{i}^{-1}\right)<\infty,
$$

holds. Also suppose that

$$
\sup _{M \geq M_{0}} M^{-\alpha} \sum_{i=1}^{\infty} L_{M, i} \cdot\left(\beta_{i} \gamma_{i}\right)^{\alpha} \cdot \mu\left(\beta_{i}\left|X_{i}\right|>M \cdot \gamma_{i}^{-1}\right)<\infty
$$


holds, where for each $M<\infty$ and $i \in \mathbb{N}, L_{M, i}$ is the bound, assumed in assumption (2.12), for the conditional probability density $\rho$ corresponding to the compact set

$$
K(M, i) \equiv\left[-3 M \cdot \beta_{i}^{-1} \gamma_{i}^{-1}, 3 M \cdot \beta_{i}^{-1} \gamma_{i}^{-1}\right] \subset \mathbb{R} .
$$

Then $\left(\mathcal{E}_{(\alpha)}, \mathcal{D}\left(\mathcal{E}_{(\alpha)}\right)\right)$ is a strictly quasi-regular Dirichlet form.

(iii) In the case where $S=\mathbb{R}^{\mathbb{N}}$ as defined by (2.3), suppose that there exists a sequence

$\left\{\gamma_{i}\right\}_{i \in \mathbb{N}}$ such that $0<\gamma_{i}, \forall i \in \mathbb{N}$, and

$$
\sup _{M \geq M_{0}} M^{-\alpha} \sum_{i=1}^{\infty} L_{M, i} \cdot \gamma_{i}^{-\alpha} \cdot \mu\left(\left|X_{i}\right|>M \cdot \gamma_{i}\right)<\infty,
$$

holds, where for each $i \in \mathbb{N}, L_{M, i}$ is the bound, assumed in assumption (2.12), for the conditional probability density $\rho$ corresponding to the compact set

$$
K(M, i) \equiv\left[-6 M \cdot \gamma_{i}, 6 M \cdot \gamma_{i}\right] \subset \mathbb{R} .
$$

Then $\left(\mathcal{E}_{(\alpha)}, \mathcal{D}\left(\mathcal{E}_{(\alpha)}\right)\right)$ is a strictly quasi-regular Dirichlet form.

Proof of Theorem 3. We use the same notations and methods as in the proof of Theorem 2. To prove the quasi-regularity we have to verify (4.12), (4.13) and (4.14) for the case where $1<\alpha<2$. To prove the strictly part of the quasi-regular Dirichlet form we have then to verify in addition that $1 \in \mathcal{D}\left(\mathcal{E}_{(\alpha)}\right)$.

Proof of (4.12), (4.13) and (4.14) for $S=l^{p}\left(\beta_{i}\right), 1 \leq p<\infty$.

Since the compactness of $D_{M}$ is independent of $\alpha$, passing through the same argument between (4.15) and (4.31), we can verify (4.12), (4.13) and (4.14) by showing that (4.30) and (4.31) hold for $1<\alpha<2$.

For each $M \in \mathbb{N}$ and $i \in \mathbb{N}$, let $a_{M, i}$ and $b_{M, i}$ be the numbers defined by (4.32):

$$
a_{M, i} \equiv \gamma_{i}^{-\frac{1}{p}}, \quad b_{M, i} \equiv 3 \gamma_{i}^{-\frac{1}{p}}
$$

we note that, by (4.24) and (4.25), the following holds:

$$
\eta_{M, i}(x)-\eta_{M, i}(y)=0, \quad \forall(x, y) \in \mathcal{N}_{M, i},
$$

where

$$
\mathcal{N}_{N, i} \equiv\left\{(x, y):|x| \leq a_{M, i},|y| \leq a_{M, i}\right\} \cup\left\{(x, y):|x|>b_{M, i},|y|>b_{M, i}\right\} .
$$

Since

$$
\begin{aligned}
& \mathbb{R}^{2} \backslash \mathcal{N}_{M, i} \\
& \quad \subset\left(\left\{(x, y):|y|>a_{M, i},|x| \leq b_{M, i}\right\} \cup\left\{(x, y):|x|>a_{M, i},|y| \leq b_{M, i}\right\}\right),
\end{aligned}
$$

for each $M \in \mathbb{N}$ and $i \in \mathbb{N}$, (cf. (4.25)), by setting

$$
K_{M, i} \equiv\left[-6 M \gamma_{i}^{-\frac{1}{p}} \beta_{i}^{-\frac{1}{p}}, 6 M \gamma_{i}^{-\frac{1}{p}} \beta_{i}^{-\frac{1}{p}}\right] \subset \mathbb{R}
$$

from (4.57) we have the following bound which is an analogue of (4.33), (4.34) and (4.35): 


$$
\begin{aligned}
& \frac{\left(\eta_{M, i}(y)-\eta_{M, i}(x)\right)^{2}}{|y-x|^{2}} I_{|y| I_{x \neq y}} \frac{1}{|y-x|^{\alpha-1}} \cdot\left(I_{K_{M, i}}(x-y)+I_{K_{M, i}^{c}}(x-y)\right) \\
& \leq\left(M^{-1} \gamma_{i}^{\frac{1}{p}} \beta_{i}^{\frac{1}{p}}\right)^{2} I_{x \neq y} \frac{1}{|y-x|^{\alpha-1}} \cdot\left(I_{K_{M, i}}(x-y)+I_{K_{M, i}^{c}}(x-y)\right) \\
& \quad \times\left(I_{|y|>a_{M, i}} I_{|x| \leq b_{M, i}}+I_{|x|>a_{M, i}} I_{|y| \leq b_{M, i}}\right),
\end{aligned}
$$

where and in the sequel, to simplify the notations, we denote, e.g., $I_{\left(-\infty,-a_{M, i}\right) \cup\left(a_{M, i}, \infty\right)}(x)$ by $I_{|x|>a_{M, i}}$, and $I_{\left[-b_{M, i}, b_{M, i}\right]}(y)$ by $I_{|y| \leq b_{M, i}}$, respectively. Next, recall the assumption (2.11) with (2.12) for a bound of the conditional probability density $\rho$ corresponding to a given compact set. In the present situation, there exists an $L_{M, i}<\infty$ and for a.e.y $\in \mathbb{R}$ with respect to the Lebesgue measure, such that

$$
\rho\left(y \mid \sigma_{i}^{c}\right) \cdot I_{\left[-b_{M, i}, b_{M, i}\right]}(y) \leq L_{M, i}, \quad \mu-\text { a.e. }
$$

Then, by applying Young's inequality we have that (cf. also (3.16))

$$
\begin{aligned}
& \sup _{x \in \mathbb{R}}\left|\int_{\mathbb{R}} \frac{I_{K_{M, i}}(x-y)}{|x-y|^{\alpha-1}} \rho\left(y \mid \sigma_{i}^{c}\right) \cdot I_{\left[-b_{M, i}, b_{M, i}\right]}(y) d y\right| \\
& \quad \leq L_{M, i}\left\|\frac{I_{K_{M, i}}}{|\cdot|^{\alpha-1}}\right\|_{L^{1}(\mathbb{R})}=\frac{2 L_{M, i}}{2-\alpha}\left(6 M \gamma_{i}^{-\frac{1}{p}} \beta_{i}^{-\frac{1}{p}}\right)^{2-\alpha}, \quad \mu-\text { a.e.. }
\end{aligned}
$$

Also by the definition of $K_{M, i}$, we have that

$$
\frac{1}{\left|y_{i}-y_{i}^{\prime}\right|^{\alpha-1}} I_{K_{M, i}^{c}}\left(y_{i}-y_{i}^{\prime}\right) \leq\left(6^{-1} M^{-1} \gamma_{i}^{\frac{1}{p}} \beta_{i}^{\frac{1}{p}}\right)^{\alpha-1}, \quad \forall y_{i}, y_{i}^{\prime} \in \mathbb{R},
$$

where we used (4.58) explicitly. For the given $f \in C_{0}^{\infty}\left(\mathbb{R}^{n} \rightarrow \mathbb{R}\right)$, let us take $M<\infty$ sufficiently large in order that (4.37) holds. Then, by (2.13) in Remark 2, (4.61) and (4.62), from (4.58), (4.59) and (4.60), the estimates corresponding to (4.38), (4.39) and (4.40), for the present value of $\alpha$, are given as follows: or $1 \leq i \leq n$,

$$
\mathcal{E}_{(\alpha)}^{(i)}\left(f_{M, k}, f_{M, k}\right) \leq \mathcal{E}_{(\alpha)}^{(i)}(u, u),
$$

and for $n+1 \leq i \leq k$ (below, for the first inequality we use (4.59), for the second and third inequality we apply (4.61) and (4.62), also, to simplify the notations, we denote, e.g., $I_{\left[-b_{M, i}, b_{M, i}\right]}\left(y_{i}\right)$ by $\left.I_{\left|y_{i}\right| \leq b_{M, i}}\right)$,

$$
\begin{aligned}
& \mathcal{E}_{(\alpha)}^{(i)}\left(f_{M, k}, f_{M, k}\right) \\
&=\int_{S}\left(f\left(x_{1}, \ldots, x_{n}\right)\right)^{2} \cdot \prod_{j \geq n+1, j \neq i}^{k}\left(\eta_{M, j}\left(x_{j}\right)\right)^{2} \prod_{l \geq k+1}\left(I_{\mathbb{R}}\left(x_{l}\right)\right)^{2} \\
& \quad \times\left\{\int_{\mathbb{R}^{2}} I_{y_{i} \neq y_{i}^{\prime}} \frac{1}{\left|y_{i}-y_{i}^{\prime}\right|^{2}}\left(\eta_{M, i}\left(y_{i}\right)-\eta_{M, i}\left(y_{i}^{\prime}\right)\right)^{2} \frac{1}{\left|y_{i}-y_{i}^{\prime}\right|^{\alpha-1}} \cdot\left(I_{K_{M, i}}\left(y_{i}-y_{i}^{\prime}\right)+I_{K_{M, i}^{c}}\left(y_{i}-y_{i}^{\prime}\right)\right)\right. \\
&\left.\quad \times \mu\left(d y_{i} \mid \sigma_{i^{c}}\right) \mu\left(d y_{i}^{\prime} \mid \sigma_{i^{c}}\right)\right\} \mu(d \mathbf{x}) \leq 2\left(M^{-1} \gamma_{i}^{\frac{1}{p}} \beta_{i}^{\frac{1}{p}}\right)^{2} \int_{S}\left(f\left(x_{1}, \ldots, x_{n}\right)\right)^{2} \prod_{j \neq i} I_{\mathbb{R}}\left(x_{j}\right) \\
& \quad \times\left\{\int_{\mathbb{R}^{2}} I_{\left|y_{i}^{\prime}\right|>a_{M, i}} \frac{1}{\left|y_{i}-y_{i}^{\prime}\right|^{\alpha-1}} \cdot\left(I_{K_{M, i}}\left(y_{i}-y_{i}^{\prime}\right)+I_{K_{M, i}^{c}}\left(y_{i}-y_{i}^{\prime}\right)\right) I_{\left|y_{i}\right| \leq b_{M, i}} \mu\left(d y_{i} \mid \sigma_{i}^{c}\right) \mu\left(d y_{i}^{\prime} \mid \sigma_{i}^{c}\right)\right\} \mu(d \mathbf{x}) \\
& \leq 2\left(M^{-1} \gamma_{i}^{\frac{1}{p}} \beta_{i}^{\frac{1}{p}}\right)^{2}\|f\|_{L^{\infty}}^{2}\left(\left\|\frac{I_{K_{M, i}}}{|\cdot|^{\alpha-1}} *\left(\rho\left(\cdot \mid \sigma_{i^{c}}\right) \cdot I_{\left|y_{i}\right| \leq b_{M, i}}\right)\right\|_{L^{\infty}(\mathbb{R})}+\left(6^{-1} M^{-1} \gamma_{i}^{\frac{1}{p}} \beta_{i}^{\frac{1}{p}}\right)^{\alpha-1}\right)
\end{aligned}
$$




$$
\begin{aligned}
& \times \int_{S}\left\{\int_{\mathbb{R}} I_{\left|y_{i}^{\prime}\right|>a_{M, i}} \mu\left(d y_{i}^{\prime} \mid \sigma_{i c}\right)\right\} \mu(d \mathbf{x}) \\
\leq & 2\left(M^{-1} \gamma_{i}^{\frac{1}{p}} \beta_{i}^{\frac{1}{p}}\right)^{2}\left\{\frac{2 L_{M, i}}{2-\alpha}\left(6 M \gamma_{i}^{-\frac{1}{p}} \beta_{i}^{-\frac{1}{p}}\right)^{2-\alpha}+\left(6^{-1} M^{-1} \gamma_{i}^{\frac{1}{p}} \beta_{i}^{\frac{1}{p}}\right)^{\alpha-1}\right\}\|f\|_{L^{\infty}}^{2} \mu\left(\left|x_{i}\right|>a_{M, i}\right), \\
= & 2\left\{\frac{2 \cdot 6^{2-\alpha} L_{M, i}}{2-\alpha}\left(M^{-1} \gamma_{i}^{\frac{1}{p}} \beta_{i}^{\frac{1}{p}}\right)^{\alpha}+6^{1-\alpha}\left(M^{-1} \gamma_{i}^{\frac{1}{p}} \beta_{i}^{\frac{1}{p}}\right)^{\alpha+1}\right\}\|f\|_{L^{\infty}}^{2} \mu\left(\left|x_{i}\right|>a_{M, i}\right),
\end{aligned}
$$

also for $k+1 \leq i$,

$$
\mathcal{E}_{(\alpha)}^{(i)}\left(f_{M, k}, f_{M, k}\right)=0,
$$

respectively. By combining (4.63), (4.64) and (4.65), from (4.32), the definition of $a_{M, i}$, for $f_{M, k}$ defined through $f \in C_{0}^{\infty}\left(\mathbb{R}^{n} \rightarrow \mathbb{R}\right), n \in \mathbb{N}$, we have

$$
\begin{aligned}
& \mathcal{E}_{(\alpha)}^{(i)}\left(f_{M, k}, f_{M, k}\right) \\
& \leq \mathcal{E}_{(\alpha)}^{(i)}(u, u)+2\left\{\frac{2 \cdot 6^{2-\alpha} L_{M, i}}{1-\alpha}\left(M^{-1} \gamma_{i}{ }^{\frac{1}{p}} \beta_{i}^{\frac{1}{p}}\right)^{\alpha}+6^{1-\alpha}\left(M^{-1} \gamma_{i}^{\frac{1}{p}} \beta_{i}^{\frac{1}{p}}\right)^{\alpha+1}\right\} \\
& \quad \times\|f\|_{L^{\infty}}^{2} \cdot \mu\left(\left|X_{i}\right|>M \gamma_{i}^{-\frac{1}{p}} \beta_{i}^{-\frac{1}{p}}\right), \quad \forall k \geq n .
\end{aligned}
$$

By (4.66), for $f_{M, k}$ defined through $f \in C_{0}^{\infty}\left(\mathbb{R}^{n} \rightarrow \mathbb{R}\right), n \in \mathbb{N}$, in order to make the discussions simple by taking $M$ satisfying both (4.37) and $M \geq M_{0}$ (cf. (4.52)), since $\mu\left(\left|X_{i}\right|>M \gamma_{i}^{-\frac{1}{p}} \beta_{i}^{-\frac{1}{p}}\right)$ is a decreasing function of $M>0$, we see that if the conditions (4.52) and (4.53) of Theorem 3-i) are satisfied, then the following analogue of (4.42) for the present value of $\alpha$ holds:

$$
\sup _{k \geq 1} \mathcal{E}_{(\alpha)}\left(f_{M, k}, f_{M, k}\right)=\sup _{k \geq 1} \sum_{i \in \mathbb{N}} \mathcal{E}_{(\alpha)}^{(i)}\left(f_{M, k}, f_{M, k}\right)<\infty .
$$

By repeating then the same discussions between (4.42) and (4.46) that have been performed for $0<\alpha \leq 1$, the proof of Theorem 3-i) is completed except for the "strictly" property, that we shall discuss below.

Proof of (4.12), (4.13) and (4.14) for $S=l^{\infty}\left(\beta_{i}\right)$, and for $S=\mathbb{R}^{\mathbb{N}}$.

The proof for these cases can be done in exactly the same way for the case $S=l_{\left(\beta_{i}\right)}^{p}$, i.e., by the discussions between (4.57) and (4.67). Namely, by changing $K_{M, i}$ of (4.58) to (cf. (4.47), (4.50))

$$
K_{M, i} \equiv\left[-6 M \cdot \beta_{i}^{-1} \gamma_{i}^{-1},-6 M \cdot \beta_{i}^{-1} \gamma_{i}^{-1}\right],
$$

and (cf. (4.49), (4.50))

$$
K_{M, i} \equiv\left[-6 M \cdot \gamma_{i},-6 M \cdot \gamma_{i}\right],
$$

respectively, similarly as in the proof of the corresponding points in Theorem 1, then through the same discussions as above, we deduce Theorem 3-ii), 3-iii), again except for the "strictly" property.

Next, let us prove that the quasi-regular Dirichlet forms $\left(\mathcal{E}_{(\alpha)}, \mathcal{D}\left(\mathcal{E}_{(\alpha)}\right)\right)$ are actually strictly quasi-regular Dirichlet forms. As remarked in the proof of Theorem 2, for this it suffices to show that $1 \in \mathcal{D}\left(\mathcal{E}_{(\alpha)}\right)$ (see Proposition V-2.15 of [69]). The proof is common for every state space $l_{\left(\beta_{i}\right)}^{p}, l_{\left(\beta_{i}\right)}^{\infty}, \mathbb{R}^{\mathbb{N}}$, and for all $1<\alpha<2$. Take $\eta \in C_{0}^{\infty}(\mathbb{R} \rightarrow \mathbb{R})$ as (4.24), namely let $\eta$ be such that $\eta(x) \geq 0,\left|\frac{d}{d x} \eta(x)\right| \leq 1$ for $x \in \mathbb{R}$, and $\eta(x)=1$ for 
$|x|<1 ; \eta(x)=0$ for $|x|>3$, and define $u_{M}\left(x_{1}, x_{2}, \ldots\right) \equiv \eta\left(x_{1} \cdot M^{-1}\right) \prod_{i>2} I_{\mathbb{R}}\left(x_{i}\right) \in$ $\mathcal{F} C_{0}^{\infty} \subset \mathcal{D}\left(\mathcal{E}_{(\alpha)}\right)$ for each $M \in \mathbb{N}$. Then, we can set $n=0$ and $k=1$ in (4.64) and (4.65) (although the original argument of (4.64) and (4.65) was performed for $n \in \mathbb{N}$, this extension to $n=0$ is admissible), and we have

$$
\begin{aligned}
\mathcal{E}_{(\alpha)}^{(1)}\left(u_{M}, u_{M}\right) \leq & \left\{\frac{6^{3-\alpha}}{2-\alpha} L_{1}\left(M^{-1} \gamma_{1}^{\frac{1}{p}} \beta_{1}^{\frac{1}{p}}\right)^{\alpha}+6^{2-\alpha}\left(M^{-1} \gamma_{1}^{\frac{1}{p}} \beta_{1}^{\frac{1}{p}}\right)^{\alpha+1}\right\} \\
& \times \mu\left(\left|x_{1}\right|>M \gamma_{1}^{-\frac{1}{p}} \beta_{1}^{-\frac{1}{p}}\right), \\
\mathcal{E}_{(\alpha)}^{(i)}\left(u_{M}, u_{M}\right)= & 0, \quad \forall i \geq 2 .
\end{aligned}
$$

By this evaluation, under the assumptions (4.53), (4.55) or (4.56), it holds that

$$
\sup _{M \in \mathbb{N}} \mathcal{E}_{(\alpha)}\left(u_{M}, u_{M}\right)<\infty .
$$

Since, $\lim _{M \rightarrow \infty} u_{M}(\mathbf{x})=1=\prod_{i>1} I_{\mathbb{R}}\left(x_{i}\right)$ pointwise, and also in $L^{2}(S ; \mu)$, from the first part of Lemma 1 we get $1 \in \mathcal{D}\left(\mathcal{E}_{(\alpha)}\right)$.

This completes the proof of Theorem 3 .

\section{Associated Markov Processes and a Standard Procedure of Application of Stochastic Quantizations on $\mathcal{S}^{\prime}$}

Let $\left(\mathcal{E}_{(\alpha)}, \mathcal{D}\left(\mathcal{E}_{(\alpha)}\right)\right), 0<\alpha<2$, be the family of strictly quasi-regular Dirichlet forms on $L^{2}(S ; \mu)$ with a state space $S$ (cf. (2.1), (2.2), (2.3)) defined by Theorems 2 and 3 . We shall first apply the general results on strictly quasi-regular Dirichlet forms and associated Markov processes (see Theorem V-2.13 and Proposition V-2.15 of [69]) to our case. By the strictly quasi-regular Dirichlet form $\left(\mathcal{E}_{(\alpha)}, \mathcal{D}\left(\mathcal{E}_{(\alpha)}\right)\right)$ there exists a properly associated $S$-valued Hunt process

$$
\mathbb{M} \equiv\left(\Omega, \mathcal{F},\left(X_{t}\right)_{t \geq 0},\left(P_{\mathbf{x}}\right)_{\mathbf{x} \in S_{\triangle}}\right)
$$

$\triangle$ is a point adjoined to $S$ as an isolated point of $S_{\triangle} \equiv S \cup\{\triangle\}$. Let $\left(T_{t}\right)_{t \geq 0}$ be the strongly continuous contraction semigroup associated with $\left(\mathcal{E}_{(\alpha)}, \mathcal{D}\left(\mathcal{E}_{(\alpha)}\right)\right)$, and $\left(p_{t}\right)_{t \geq 0}$ be the corresponding transition semigroup of kernels of the Hunt process $\left(X_{t}\right)_{t \geq 0}$, then for any $u \in \mathcal{F} C_{0}^{\infty} \subset \mathcal{D}\left(\mathcal{E}_{(\alpha)}\right)$ the following holds:

$$
\frac{d}{d t} \int_{S}\left(p_{t} u\right)(\mathbf{x}) \mu(d \mathbf{x})=\frac{d}{d t}\left(T_{t} u, 1\right)_{L^{2}(S ; \mu)}=\mathcal{E}_{(\alpha)}\left(T_{t} u, 1\right)=0 .
$$

By this, we see that

$$
\int_{S}\left(p_{t} u\right)(\mathbf{x}) \mu(d \mathbf{x})=\int_{S} u(\mathbf{x}) \mu(d \mathbf{x}), \quad \forall t \geq 0, \quad \forall u \in \mathcal{F} C_{0}^{\infty},
$$

and hence, by the density of $\mathcal{F} C_{0}^{\infty}$ in $L^{2}(S ; \mu)$,

$$
\int_{S} P_{\mathbf{x}}\left(X_{t} \in B\right) \mu(d \mathbf{x})=\mu(B), \quad \forall B \in \mathcal{B}(S), \quad \forall t \geq 0 .
$$

Thus, we have proven the following Theorem 4. 
Theorem 4. Let $0<\alpha<2$, and let $\left(\mathcal{E}_{(\alpha)}, \mathcal{D}\left(\mathcal{E}_{(\alpha)}\right)\right)$ be a strictly quasi-regular Dirichlet form on $L^{2}(S ; \mu)$ that is defined through Theorems 2 or 3 . Then to $\left(\mathcal{E}_{(\alpha)}, \mathcal{D}\left(\mathcal{E}_{(\alpha)}\right)\right)$, there exists a properly associated $S$-valued Hunt process ( $c f$. Definitions IV-1.5, 1.8 and 1.13 of [69] for its precise definition) $\mathbb{M}$ defined by (5.1), the invariant measure of which is $\mu(c f .(5.4))$.

We introduce below a standard procedure of application of Theorems 1, 2, 3 and 4 to the problem of stochastic quantizations of Euclidean quantum fields, by means of the Hunt processes in Theorem 4. Mostly by the term stochastic quantization one understands methods to construct diffusion type Markov processes (with continuous trajectories) which possess as their invariant measures given probability measures $m$ associated to a given physical system (e.g., describing a statistical mechanical system or a quantum system, cf. [76] and [25-27],), with references therein. Often it is interesting to consider their analogues where $\mathbb{R}^{d}$ is replaced by the $d$-dimensional torus $\mathbb{T}^{d}$ (since they can be used to approximate the (Euclidean quantum) fields on $\mathbb{R}^{d}$ ). Moreover the original formulation of stochastic quantization can be naturally extended in the sense of asking for Markov processes having $m$ as invariant measure. Here, we realize such Markov processes by the Hunt processes associated with $\left(\mathcal{E}_{(\alpha)}, \mathcal{D}\left(\mathcal{E}_{(\alpha)}\right)\right)$ given by theorem 4 , which are infinite dimensional analogues of $\alpha$-stable processes on finite dimensional space (cf. (2.8), (2.9) and (2.10), for the definition of the corresponding forms $\mathcal{E}_{(\alpha)}$ ). At the end of the present section, examples based on Euclidean free fields, or the fields associated with the $\Phi_{2}^{4}$ and $\Phi_{3}^{4}$ models are indicated for $0<\alpha \leq 1$.

Remark 5. Here we only briefly indicate the way these applications are obtained, detailed considerations on the individual examples of the Euclidean quantum field models, e.g., the fields constructed from convoluted generalized white noise (cf. [11]), and the free Euclidean field in all $\mathbb{R}^{d}$, the $P(\phi)_{2}$ field, the 2-dimensional fields with trigonometric and exponential potentials, and of other random fields, will be carried out in a subsequent paper ([A,Kaga,KawaYaha,Y 2021] part 2: the applications).

Euclidean (scalar) quantum fields are expressed as random fields on $\mathcal{S}^{\prime} \equiv \mathcal{S}^{\prime}\left(\mathbb{R}^{d} \rightarrow\right.$ $\mathbb{R})$, or $\mathcal{S}^{\prime}\left(\mathbb{T}^{d} \rightarrow \mathbb{R}\right)$, the Schwartz space of real tempered distributions on the Euclidean space $\mathbb{R}^{d}$, or the $d$-dimensional torus $\mathbb{T}^{d}$, with $d \geq 1$ a given space-time dimension, respectively. Hence, each Euclidean quantum field is taken as a probability space $\left(\mathcal{S}^{\prime}, \mathcal{B}\left(\mathcal{S}^{\prime}\right), v\right)$, where $\mathcal{B}\left(\mathcal{S}^{\prime}\right)$ is the Borel $\sigma$-field of $\mathcal{S}^{\prime}$ and $\nu$ is a Borel probability measure on $\mathcal{S}^{\prime}$ (on $\mathbb{R}^{d}$, or on $\mathbb{T}^{d}$, invariant under the rigid natural transformations of $\mathbb{R}^{d}$, or $\mathbb{T}^{d}$, respectively, that correspond to the Euclidean group). In the construction the standard theorem through which probability measures $v$ are determined is the Bochner-Minlos theorem (cf. e.g., Section 3.2 of [58]).

Let us first recall the Bochner-Minlos theorem stated in a general framework. Let $E$ be a nuclear space (cf., e.g., Chapters 47-51 of [91]). Suppose in particular that $E$ is a countably Hilbert space, $E$ is characterized by a sequence of Hilbert norms \|\|$_{n}$, $n \in \mathbb{N} \cup\{0\}$ such that \|\|$_{0}<\|\|_{1}<\cdots<\|\|_{n}<\cdots$. Let $E_{n}$ be the completion of $E$ with respect to the norm \|\|$_{n}$, then by definition $E=\bigcap_{n \geq 0} E_{n}$ and $E_{0} \supset E_{1} \supset \cdots \supset$ $E_{n} \supset \cdots$. Define

$$
E_{n}^{*} \equiv \text { the dual space of } E_{n} \text {, and assume the identification } E_{0}^{*}=E_{0},
$$

then we have

$$
E \subset \cdots \subset E_{n+1} \subset E_{n} \subset \cdots \subset E_{0}=E_{0}^{*} \subset \cdots \subset E_{n}^{*} \subset E_{n+1}^{*} \subset \cdots \subset E^{*} .
$$


Since $E$ is a nuclear space, for any $m \in \mathbb{N} \cup\{0\}$ there exists an $n \in \mathbb{N} \cup\{0\}, n>m$, such that the (canonical) injection $T_{m}^{n}: E_{n} \rightarrow E_{m}$ is a trace class (nuclear class) positive operator. The Bochner-Minlos theorem is given as follows:

Theorem 5 (Bochner-Minlos Theorem). Let $C(\varphi), \varphi \in E$, be a complex valued function on E such that

(i) $C(\varphi)$ is continuous with respect to the norm $\|\cdot\|_{m}$ for some $m \in \mathbb{N} \cup\{0\}$;

(ii) (positive definiteness) for any $k \in \mathbb{N}$,

$$
\sum_{i, j=1}^{k} \bar{\alpha}_{i} \alpha_{j} C\left(\varphi_{i}-\varphi_{j}\right) \geq 0, \quad \forall \alpha_{i} \in \mathbb{C}, \forall \varphi_{i} \in E, i=1, \ldots, k
$$

(where $\bar{\alpha}$ means complex conjugate of $\alpha$ ).

(iii) (normalization) $C(0)=1$. Then, there exists a unique Borel probability measure $v$ on $E^{*}$ such that

$$
C(\varphi)=\int_{E^{*}} e^{i<\phi, \varphi>} v(d \phi), \quad \varphi \in E .
$$

Moreover, for all $n>m$, if the (canonical) injection $T_{m}^{n}: E_{n} \rightarrow E_{m}$ is a HilbertSchmidt operator, then the support of $v$ is in $E_{n}^{*}$, where $\left.<\phi, \varphi>=E^{*}<\phi, \varphi\right\rangle_{E}$ is the dualization between $\phi \in E^{*}$ and $\varphi \in E$.

Remark 6. The assumption on the continuity of $C(\varphi)$ on $E$ given in i) of the above Theorem 5 can be replaced by the continuity of $C(\varphi)$ at the origin in $E$, which is equivalent to i) under the assumption that $C(\varphi)$ satisfies ii) and iii) in Theorem 5 (cf. e.g., [61]). Namely, under the assumption of ii) and iii), the following is equivalent to i): For any $\epsilon>0$ there exists a $\delta>0$ such that

$$
|C(\varphi)-1|<\epsilon, \quad \forall \varphi \in E \text { with }\|\varphi\|_{m}<\delta .
$$

This can be seen as follows: Assume that ii) and iii) hold. For ii), let $k=3, \alpha_{1}=\alpha$, $\alpha_{2}=-\alpha, \alpha_{3}=\beta, \varphi_{1}=0, \varphi_{2}=\varphi$ and $\varphi_{3}=\psi+\varphi$, (for any $\alpha, \beta \in \mathbb{C}$, and any $\varphi, \psi \in E$ ) then by the assumption ii), the positive definiteness of $C$, we have

$$
\begin{aligned}
& \alpha \bar{\alpha} \cdot(2 C(0)-C(\varphi)-C(-\varphi)) \\
& \quad+\alpha \bar{\beta} \cdot(C(-\psi-\varphi)-C(-\psi))+\bar{\alpha} \beta \cdot(C(\psi+\varphi)-C(\psi))+\beta \bar{\beta} \cdot C(0) \geq 0 .
\end{aligned}
$$

By making use of the fact that $C(-\varphi)=\overline{C(\varphi)}$, which follows from ii), and the assumption iii), from the above inequality we have

$$
0 \leq \operatorname{det}\left(\begin{array}{cc}
2-C(\varphi)-\overline{C(\varphi)} & \overline{C(\psi+\varphi)-C(\psi)} \\
C(\psi+\varphi)-C(\psi) & 1
\end{array}\right) .
$$

From this it follows that

$$
|C(\psi+\varphi)-C(\psi)|^{2} \leq 2|C(\varphi)-1| .
$$


By making use of the support property of $v$ by means of the Hilbert-Schmidt operators given by Theorem 5, we can present a framework by which Theorems 1, 2, 3 and 4 can be applied to the stochastic quantization of Euclidean quantum fields.

We first define an adequate countably Hilbert nuclear space $\mathcal{H}_{0} \supset \mathcal{S}\left(\mathbb{R}^{d} \rightarrow \mathbb{R}\right) \equiv$ $\mathcal{S}\left(\mathbb{R}^{d}\right)$, for a given $d \in \mathbb{N}$ (cf., e.g.,Appendix A.3 of [58], Appendix 5 of [59] for the framework on nuclear countable Hilbert space). Let

$$
\mathcal{H}_{0} \equiv\left\{f:\|f\|_{\mathcal{H}_{0}}=\left((f, f)_{\mathcal{H}_{0}}\right)^{\frac{1}{2}}<\infty, \quad f: \mathbb{R}^{d} \rightarrow \mathbb{R}, \text { measurable }\right\} \supset \mathcal{S}\left(\mathbb{R}^{d}\right),
$$

where

$$
(f, g)_{\mathcal{H}_{0}} \equiv(f, g)_{L^{2}\left(\mathbb{R}^{d}\right)}=\int_{\mathbb{R}^{d}} f(x) g(x) d x .
$$

Let

$$
\begin{aligned}
H & \equiv\left(|x|^{2}+1\right)^{\frac{d+1}{2}}(-\Delta+1)^{\frac{d+1}{2}}\left(|x|^{2}+1\right)^{\frac{d+1}{2}}, \\
H^{-1} & \equiv\left(|x|^{2}+1\right)^{-\frac{d+1}{2}}(-\Delta+1)^{-\frac{d+1}{2}}\left(|x|^{2}+1\right)^{-\frac{d+1}{2}},
\end{aligned}
$$

be the pseudo differential operators on $\mathcal{S}^{\prime}\left(\mathbb{R}^{d} \rightarrow \mathbb{R}\right) \equiv \mathcal{S}^{\prime}\left(\mathbb{R}^{d}\right)$ with $\Delta$ the $d$-dimensional Laplace operator. Note that $H^{-1}$ is strictly positive bounded and symmetric, hence selfadjoint in $L^{2}\left(\mathbb{R}^{d}\right)$. For each $n \in \mathbb{N}$, define

$$
\mathcal{H}_{n} \equiv \text { the completion of } \mathcal{S}\left(\mathbb{R}^{d}\right) \text { with respect to the norm }\|f\|_{n}, f \in \mathcal{S}\left(\mathbb{R}^{d}\right),
$$

where $\|f\|_{n}^{2} \equiv(f, f)_{n}$ (in the case where $n=1$, to denote the $\mathcal{H}_{1}$ norm we use the exact notation \|\|$_{\mathcal{H}_{1}}$, in order to avoid a confusion with the notation of some $L^{1}$ or $l^{1}$ norms) with the corresponding scalar product

$$
(f, g)_{n}=\left(H^{n} f, H^{n} g\right)_{\mathcal{H}_{0}}, \quad f, g \in \mathcal{S}\left(\mathbb{R}^{d}\right) .
$$

Moreover we define, for $n \in \mathbb{N}$

$$
\mathcal{H}_{-n} \equiv \text { the completion of } \mathcal{S}\left(\mathbb{R}^{d}\right) \text { with respect to the norm }\|f\|_{-n}, f \in \mathcal{S}\left(\mathbb{R}^{d}\right),
$$

where $\|f\|_{-n}^{2} \equiv(f, f)_{-n}$, with

$$
(f, g)_{-n}=\left(\left(H^{-1}\right)^{n} f,\left(H^{-1}\right)^{n} g\right)_{\mathcal{H}_{0}}, \quad f, g \in \mathcal{S}\left(\mathbb{R}^{d}\right) .
$$

Then obviously, for $f \in \mathcal{S}\left(\mathbb{R}^{d}\right)$,

$$
\|f\|_{n} \leq\|f\|_{n+1}, \quad\|f\|_{-n-1} \leq\|f\|_{-n},
$$

and by taking the inductive limit and setting $\mathcal{H}=\bigcap_{n \in \mathbb{N}} \mathcal{H}_{n}$, we have the following inclusions:

$$
\mathcal{H} \subset \cdots \subset \mathcal{H}_{n+1} \subset \mathcal{H}_{n} \subset \cdots \subset \mathcal{H}_{0} \subset \cdots \subset \mathcal{H}_{-n} \subset \mathcal{H}_{-n-1} \subset \cdots \subset \mathcal{H}^{*}
$$


The (topological) dual space of $\mathcal{H}_{n}$ is $\mathcal{H}_{-n}, n \in \mathbb{N}$.

By the operator $H^{-1}$ given by (5.8) on $\mathcal{S}\left(\mathbb{R}^{d}\right)$ we can define, on each $\mathcal{H}_{n}, n \in \mathbb{N}$, the bounded symmetric (hence self-adjoint) operators

$$
\left(H^{-1}\right)^{k}, \quad k \in \mathbb{N} \cup\{0\}
$$

(we use the same notations for the operators on $\mathcal{S}\left(\mathbb{R}^{d}\right)$ and on $\left.\mathcal{H}_{n}\right)$. Hence, for the canonical injection

$$
T_{n}^{n+k}: \mathcal{H}_{n+k} \longrightarrow \mathcal{H}_{n}, \quad k, n \in \mathbb{N} \cup\{0\},
$$

it holds that

$$
\left\|T_{n}^{n+k} f\right\|_{n}=\left\|\left(H^{-1}\right)^{k} f\right\|_{\mathcal{H}_{0}}, \quad \forall f \in \mathcal{H}_{n+k},
$$

where by a simple calculation by means of the Fourier transform, and by Young's inequality, we see that for each $n \in \mathbb{N} \cup\{0\}, H^{-1}$ on $\mathcal{H}_{n}$ is a Hilbert-Schmidt operator and hence $\left(H^{-1}\right)^{2}$ on $\mathcal{H}_{n}$ is a trace class operator.

Now, by applying to the strictly positive self-adjoint Hilbert-Schmidt (hence compact) operator $H^{-1}$, on $\mathcal{H}_{0}=L^{2}\left(\mathbb{R}^{d} \rightarrow \mathbb{R}\right.$ ) the Hilbert-Schmidt theorem (cf., e.g., Theorem VI 16, Theorem VI 22 of [79]) we have that there exists an orthonormal base (O.N.B.) $\left\{\varphi_{i}\right\}_{i \in \mathbb{N}}$ of $\mathcal{H}_{0}$ such that

$$
H^{-1} \varphi_{i}=\lambda_{i} \varphi_{i}, \quad i \in \mathbb{N},
$$

where $\left\{\lambda_{i}\right\}_{i \in \mathbb{N}}$ are the corresponding eigenvalues such that

$$
0<\cdots<\lambda_{2}<\lambda_{1} \leq 1 \text {, which satisfy } \sum_{i \in \mathbb{N}}\left(\lambda_{i}\right)^{2}<\infty \text {, i.e., }\left\{\lambda_{i}\right\}_{i \in \mathbb{N}} \in l^{2},
$$

and $\left\{\varphi_{i}\right\}_{i \in \mathbb{N}}$ is indexed adequately corresponding to the finite multiplicity of each $\lambda_{i}$, $i \in \mathbb{N}$. By the definition (5.9), (5.10), (5.11) and (5.12) (cf. also (5.15)), for each $n \in \mathbb{N} \cup\{0\}$,

$$
\left\{\left(\lambda_{i}\right)^{n} \varphi_{i}\right\}_{i \in \mathbb{N}} \text { is an O.N.B. of } \mathcal{H}_{n}
$$

and

$$
\left\{\left(\lambda_{i}\right)^{-n} \varphi_{i}\right\}_{i \in \mathbb{N}} \text { is an O.N.B. of } \mathcal{H}_{-n}
$$

Thus, by denoting $\mathbb{Z}$ the set of integers, by the Fourier series expansion of functions in $\mathcal{H}_{m}, m \in \mathbb{Z}$ (cf. (5.9)-(5.12)), such that for $f \in \mathcal{H}_{m}$, we have

$$
f=\sum_{i \in \mathbb{N}} a_{i}\left(\lambda_{i}^{m} \varphi_{i}\right), \quad \text { with } a_{i} \equiv\left(f,\left(\lambda_{i}^{m} \varphi_{i}\right)\right)_{m}, i \in \mathbb{N}
$$

(in particular for $f \in \mathcal{S}\left(\mathbb{R}^{d}\right) \subset \mathcal{H}_{m}$, it holds that $\left.a_{i}=\lambda_{i}^{-m}\left(f, \varphi_{i}\right) \mathcal{H}_{0}\right)$. Moreover we have

$$
\sum_{i \in \mathbb{N}} a_{i}^{2}=\|f\|_{m}^{2}
$$


that yields an isometric isomorphism $\tau_{m}$ for each $m \in \mathbb{Z}$ such that

$$
\tau_{m}: \mathcal{H}_{m} \ni f \longmapsto\left(\lambda_{1}^{m} a_{1}, \lambda_{2}^{m} a_{2}, \ldots\right) \in l_{\left(\lambda_{i}^{-2 m}\right)}^{2},
$$

where $l_{\left(\lambda_{i}^{-2 m}\right)}^{2}$ is the weighted $l^{2}$ space defined by (2.1) with $p=2$, and $\beta_{i}=\lambda_{i}^{-2 m}$. Precisely, for $f=\sum_{i \in \mathbb{N}} a_{i}\left(\lambda_{i}^{m} \varphi_{i}\right) \in \mathcal{H}_{m}$ and $g=\sum_{i \in \mathbb{N}} b_{i}\left(\lambda_{i}^{m} \varphi_{i}\right) \in \mathcal{H}_{m}$, with $a_{i} \equiv$ $\left(f,\left(\lambda_{i}^{m} \varphi_{i}\right)\right)_{m}, b_{i} \equiv\left(g,\left(\lambda_{i}^{m} \varphi_{i}\right)\right)_{m}, i \in \mathbb{N}$, by $\tau_{m}$ the following holds (cf. (5.19) and $(5.20))$ :

$$
(f, g)_{m}=\sum_{i \in \mathbb{N}} a_{i} \cdot b_{i}=\sum_{i \in \mathbb{N}} \lambda_{i}^{-m}\left(\lambda_{i}^{m} a_{i}\right) \cdot \lambda_{i}^{-m}\left(\lambda_{i}^{m} b_{i}\right)=\left(\tau_{m} f, \tau_{m} g\right)_{l_{\left(\lambda_{i}^{-2 m}\right)}^{2}} .
$$

By the map $\tau_{m}$ we can identify, in particular, the two systems of Hilbert spaces given by (5.23) and (5.24) through the following diagram:

$$
\begin{aligned}
& \cdots \mathcal{H}_{2} \subset \mathcal{H}_{1} \subset \mathcal{H}_{0}=L^{2}\left(\mathbb{R}^{d}\right) \subset \mathcal{H}_{-1} \subset \mathcal{H}_{-2} \quad \cdots, \\
& \text { \| } \quad \text { | } \quad \text { | } \quad \text { ॥ } \\
& \cdots l_{\left(\lambda_{i}^{-4}\right)}^{2} \subset l_{\left(\lambda_{i}^{-2}\right)}^{2} \subset \quad l^{2} \subset l_{\left(\lambda_{i}^{2}\right)}^{2} \subset l_{\left(\lambda_{i}^{4}\right)}^{2} \cdots .
\end{aligned}
$$

Example 1. (The Euclidean free fields) Let $\nu_{0}$ be the Euclidean free field probability measure on $\mathcal{S}^{\prime} \equiv \mathcal{S}^{\prime}\left(\mathbb{R}^{d}\right)$. Then, the (generalized) characteristic function $C(\varphi)$ of $v_{0}$ in Theorem 5 is given by

$$
C(\varphi)=\exp \left(-\frac{1}{2}\left(\varphi,\left(-\Delta+m_{0}^{2}\right)^{-1} \varphi\right)_{L^{2}\left(\mathbb{R}^{d}\right)}\right), \quad \text { for } \varphi \in \mathcal{S}\left(\mathbb{R}^{d} \rightarrow \mathbb{R}\right) .
$$

Equivalently, $v_{0}$ is a centered Gaussian probability measure on $\mathcal{S}^{\prime}$, the covariance of which is given by

$$
\begin{aligned}
& \int_{\mathcal{S}^{\prime}}<\phi, \varphi_{1}>\cdot<\phi, \varphi_{1}>v_{0}(d \phi)=\left(\varphi_{1},\left(-\Delta+m_{0}^{2}\right)^{-1} \varphi_{2}\right)_{L^{2}\left(\mathbb{R}^{d}\right)}, \\
& \quad \text { for any } \varphi_{1}, \varphi_{2} \in \mathcal{S}\left(\mathbb{R}^{d} \rightarrow \mathbb{R}\right),
\end{aligned}
$$

where $\Delta$ is the $d$-dimensional Laplace operator and $m_{0}>0$ (for $d \geq 3$, we can allow also $m_{0}=0$ ) is a given mass for this scalar field (the coordinate process to $v_{0}$ ). By (5.25), the functional $C(\varphi)$ is continuous with respect to the norm of the space $\mathcal{H}_{0}=L^{2}\left(\mathbb{R}^{d}\right)$, and the kernel of $\left(-\Delta+m_{0}^{2}\right)^{-1}$, which is the Fourier inverse transform of $\left(|\xi|^{2}+m_{0}^{2}\right)^{-1}$, $\xi \in \mathbb{R}^{d}$, is explicitly given by Bessel functions (cf., e.g., section 2-5 of [72]). Then, by Theorem 5 and (5.16) the support of $\nu_{0}$ can be taken to be in the Hilbert spaces $\mathcal{H}_{-n}$, $n \geq 1$ (cf. (5.23) and (5.24)).

Let us apply Theorems 1, 2 and 4 with $p=\frac{1}{2}$ to this random field. For a clarity of the discussion, we start the consideration from the case where $\alpha=1$. Then, we shall state the corresponding results for the cases where $0<\alpha<1$.

Now, we take $v_{0}$ as a Borel probability measure on $\mathcal{H}_{-2}$. By (5.22), (5.23) and (5.24), by taking $m=-2, \tau_{-2}$ defines an isometric isomorphism such that

$$
\tau_{-2}: \mathcal{H}_{-2} \ni f \longmapsto\left(\lambda_{1}^{-2} a_{1}, \lambda_{1}^{-2} a_{2}, \ldots\right) \in l_{\left(\lambda_{i}^{4}\right)}^{2},
$$




$$
\text { with } a_{i} \equiv\left(f, \lambda_{i}^{-2} \varphi_{i}\right)_{-2}, \quad i \in \mathbb{N} \text {. }
$$

Define a probability measure $\mu$ on $l_{\left(\lambda_{i}^{4}\right)}^{2}$ such that

$$
\mu(B) \equiv \nu_{0} \circ \tau_{-2}^{-1}(B) \text { for } B \in \mathcal{B}\left(l_{\left(\lambda_{i}^{4}\right)}^{2}\right)
$$

We set $S=l_{\left(\lambda_{i}^{4}\right)}^{2}$ in Theorems 1,2 and 4, then it follows that the weight $\beta_{i}$ satisfies $\beta_{i}=\lambda_{i}^{4}$. We can take $\gamma_{i}^{-\frac{1}{2}}=\lambda_{i}$ in Theorem 2-i) with $p=2$, then, from (5.18) we have

$$
\sum_{i=1}^{\infty} \beta_{i} \gamma_{i} \cdot \mu\left(\beta_{i}^{\frac{1}{2}}\left|X_{i}\right|>M \cdot \gamma_{i}^{-\frac{1}{2}}\right) \leq \sum_{i=1}^{\infty} \beta_{i} \gamma_{i}=\sum_{i=1}^{\infty}\left(\lambda_{i}\right)^{2}<\infty
$$

(5.28) shows that the condition (4.3) holds for $p=2$ and $\alpha=1$. Also, as has been mentioned above, since $v_{0}\left(\mathcal{H}_{-n}\right)=1$, for any $n \geq 1$, we have

$$
\begin{aligned}
& 1=\nu_{0}\left(\mathcal{H}_{-1}\right)=\mu\left(l_{\left(\lambda_{i}^{2}\right)}^{2}\right)=\mu\left(\bigcup_{M \in \mathbb{N}}\left\{\left|X_{i}\right| \leq M \beta_{i}^{-\frac{1}{2}} \gamma_{i}^{-\frac{1}{2}}, \forall i \in \mathbb{N}\right\}\right), \\
& \quad \text { for } \beta_{i}=\lambda_{i}^{4}, \gamma_{i}^{-\frac{1}{2}}=\lambda_{i}
\end{aligned}
$$

This shows that the condition (4.4) is satisfied for $p=2$.

Thus, by Theorem 2-i) and Theorem 4, for $\alpha=1$, there exists an $l_{\left(\left(\lambda_{i}\right)^{4}\right)}^{2}$-valued Hunt process $\mathbb{M} \equiv\left(\Omega, \mathcal{F},\left(X_{t}\right)_{t \geq 0},\left(P_{\mathbf{x}}\right)_{\mathbf{x} \in S_{\Delta}}\right)$, associated to the non-local Dirichlet form $\left(\mathcal{E}_{(\alpha)}, \mathcal{D}\left(\mathcal{E}_{(\alpha)}\right)\right)$. We can now define an $\mathcal{H}_{-2}$-valued process $\left(Y_{t}\right)_{t \geq 0}$ such that

$$
\left(Y_{t}\right)_{t \geq 0} \equiv\left(\tau_{-2}^{-1}\left(X_{t}\right)\right)_{t \geq 0} .
$$

Equivalently, by (5.27) for $X_{t}=\left(X_{1}(t), X_{2}(t), \ldots\right) \in l_{\left(\lambda_{i}^{4}\right)}^{2}, P_{\mathbf{x}}-$ a.e., by setting $A_{i}(t)$ such that $A_{i}(t) \equiv \lambda_{i}^{2} X_{i}(t)$ (cf. (5.21) and (5.22)), we see that $Y_{t}$ is also given by

$$
Y_{t}=\sum_{i \in \mathbb{N}} A_{i}(t)\left(\lambda_{i}^{-2} \varphi_{i}\right)=\sum_{i \in \mathbb{N}} X_{i}(t) \varphi_{i} \in \mathcal{H}_{-2}, \quad \forall t \geq 0, P_{\mathbf{x}}-\text { a.e.. }
$$

By (5.4) and (5.27), $Y_{t}$ is an $\mathcal{H}_{-2}$-valued Hunt process that is a stochastic quantization (according to the definition we gave to this term) with respect to the non-local Dirichlet form $\left(\tilde{\mathcal{E}}_{(\alpha)}, \mathcal{D}\left(\tilde{\mathcal{E}}_{(\alpha)}\right)\right)$ on $L^{2}\left(\mathcal{H}_{-2}, v_{0}\right)$, that is defined through $\left(\mathcal{E}_{(\alpha)}, \mathcal{D}\left(\mathcal{E}_{(\alpha)}\right)\right)$, by making use of $\tau_{-2}$. This holds for $\alpha=1$.

For the cases where $0<\alpha<1$ and $p=2$, we can also apply Theorems 1, 2 and 4, and then have the corresponding result to (5.29). For this purpose we have only to notice that for $\alpha \in(0,1)$ if we take $v_{0}$ as a Borel probability measure on $\mathcal{H}_{-3}$, and set $S \equiv l_{\left(\lambda_{i}^{6}\right)}^{2}$, $\beta_{i} \equiv \lambda_{i}^{6}, \gamma_{i} \equiv \lambda_{i}$, and define

$$
\tau_{-3}: \mathcal{H}_{-3} \ni f \longmapsto\left(\lambda_{1}^{-3} a_{1}, \lambda_{1}^{-3} a_{2}, \ldots\right) \in l_{\left(\lambda_{i}^{6}\right)}^{2}, \text { with } a_{i} \equiv\left(f, \lambda_{i}^{-3} \varphi_{i}\right)_{-3}, \quad i \in \mathbb{N},
$$


(cf. (5.22), (5.23), (5.24) and (5.27)), then

$$
\sum_{i=1}^{\infty}\left(\beta_{i} \gamma_{i}\right)^{\frac{\alpha+1}{2}}=\sum_{i=1}^{\infty}\left(\lambda_{i}\right)^{2(\alpha+1)}<\infty
$$

As a consequence, for $\alpha \in(0,1)$, we adopt here the same formulation as will be given in the next example, Example 2 (cf. (5.58)-(5.62)), we then see that by this setting (4.3) and (4.4) also hold (cf. (5.28) and the formula given below (5.28)), and have an analogue of (5.29).

The case $1<\alpha<2$ requires a separate consideration, see [19]. The diffusion case $\alpha=2$ was already discussed in [29,31] (and references therein).

Remark 7 (A structural property of Gaussian random fields). The Euclidean free field considered in Example 1 is a Gaussian field, which possesses a simple mathematical structure as follows: Let $D(x-y)$ denotes the integral kernel corresponding to the pseudo differential operator $\left(-\Delta+m_{0}\right)^{-1}$ on $\mathcal{S}\left(\mathbb{R}^{d} \rightarrow \mathbb{R}\right)$ considered in Example 1 (cf. (5.26) and the explanation follwing to (5.26)). Then $\left(\varphi_{1},\left(-\Delta+m_{0}\right)^{-1} \varphi_{2}\right)_{L^{2}\left(\mathbb{R}^{d}\right)}=$ $\int_{\mathbb{R}^{d} \times \mathbb{R}^{d}} \varphi_{1}(x) D(x-y) \varphi_{2}(y) d x d y . D(x-y)$ is often refered to as the free propagator. For a notational simplicity, let us denote $\int_{\mathbb{R}^{d} \times \mathbb{R}^{d}} \varphi_{i}(x) D(x-y) \varphi_{j}(y) d x d y, \varphi_{i} \in$ $\mathcal{S}\left(\mathbb{R}^{d} \rightarrow \mathbb{R}\right), i=1, \ldots, n, n \in \mathbb{N}$, by $a_{i, j}$. Then it holds that (cf. (5.26)), for $n \in \mathbb{N}$,

$$
\int_{\mathcal{S}^{\prime}} \prod_{i=1}^{2 n}<\phi, \varphi_{i}>v_{0}(d \phi)=\sum_{\text {pairing }} \prod a_{i, j}, \quad \int_{\mathcal{S}^{\prime}} \prod_{i=1}^{2 n-1}<\phi, \varphi_{i}>v_{0}(d \phi)=0,
$$

where $\sum_{\text {pairing }}$ denotes the sum of all the combinations of the distinguished pairings in $\{1, \ldots, 2 n\}$, and $\prod a_{i, j}$ is the product of $a_{i, j}$ characterized by each pairing. Precisely, each pairing is determined as follows: Firstly, as the first pair we choose two indices from $\{1, \ldots, 2 n\}$, secondly, as the second pair we choose two indices from $\{1, \ldots, 2 n\} \backslash\{$ previously chosen indices $\}$, thirdly, as the third pair we choose two indices from $\{1, \ldots, 2 n\} \backslash\{$ previously chosen indices $\}$, and so on. Among all the pairings gotten through the above procedure, as a consequence, we have $(2 n-1)$ !! number of distinguished pairings, where

$$
(2 n-1) ! ! \equiv(2 n-1) \cdot(2 n-3) \cdots 3 \cdot 1 .
$$

Thus, as a special case, it holds that

$$
\int_{\mathcal{S}^{\prime}}<\phi, \varphi>^{2 n} \nu_{0}(d \phi)=(2 n-1) ! !\left(\left(\varphi,\left(-\Delta+m_{0}\right)^{-1} \varphi\right)_{L^{2}\left(\mathbb{R}^{d}\right)}\right)^{n}, \quad \varphi \in \mathcal{S}\left(\mathbb{R}^{d} \rightarrow \mathbb{R}\right) .
$$

These properties expressing even moments of mean zero Gaussian random field with sum of products of second moments are characteristic of the fields being Gaussian.

Example 2. (The Euclidean $\Phi_{1}^{4}, \Phi_{2}^{4}$ and $\Phi_{3}^{4}$ fields) As a rough explanation, the Euclidean $\Phi_{d}^{4}, d=1,2,3$ fields are probability measures on $\mathcal{S}^{\prime}\left(\mathbb{R}^{d} \rightarrow \mathbb{R}\right)$, that describe Euclidean invariant random fields having self interactions with fourth power, and are defined by adding corresponding interaction terms to the Euclidean free fields on $\mathcal{S}^{\prime}\left(\mathbb{R}^{d} \rightarrow \mathbb{R}\right)$, $d=1,2$, 3. (cf., e.g., [63] for the case $d=1$; [52,89] for fields in the case $d=2$ with general polynomial interactions called $P(\Phi)_{2}$ (Euclidean) field, for trigonometric and exponential see also, [14-16,18,36,93] and references therein; for the $\Phi_{3}^{4}$-field see the 
references in [52] and [22,56], [A.Kusuoka-sei 2021], [54]). There are several strategies (to cope with the singularities of the fields) through which the $\Phi_{d}^{4}, d=2,3$, Euclidean fields are constructed. A standard construction strategy is to start from (continuum) random fields on $\mathcal{S}^{\prime}\left(\mathbb{R}^{d} \rightarrow \mathbb{R}\right.$ ), associated with a bounded region in $\mathbb{R}^{d}$ ( or a torus $\mathbb{T}^{d}$ ) and then expanding to $\mathbb{R}^{d}$, or alternative, to start from random fields on a lattice $(\epsilon \mathbb{Z})^{d}$ (with the lattice spacing $\epsilon>0$, which subsequently tends to 0 , i.e., taking a continuum limit). In both cases, for $d=2,3$, renormalization counter terms are required for a non trivial limit.

The stochastic quantization of $\Phi_{d}^{4}, d=2,3$, is also not a trivial problem. In fact for $d=2$ it was obtained first by the local Dirichlet form method in the 90's (cf. [29-31]) and in the sense of strong solutions in [Da Prato,Debussche] see also [82]. For $d=3$ it has been an open problem until the publication [56] for the diffusion case on $\Phi_{3}^{4}$ Euclidean field on the 3-dimensional torus $\mathbb{T}^{3}$. After this, several publications obtained the stochastic quantization on $\mathbb{R}^{3}$ (see [54] and also references in [22] and [23]). The methods of the present paper show that a stochastic quantization of $\Phi_{3}^{4}$ Euclidean field on $\mathbb{R}^{3}$ can also be realized by non-local Hunt processes. In order to understand the difficulty of the stochastic quantization program, which is caused by singularities of the $\Phi_{d}^{4}$ Euclidean field measures, we briefly recall above construction procedures.

The first one is as follows: Let $\nu_{0}$ be the Euclidean free field measure on $\mathcal{S}^{\prime} \equiv$ $\mathcal{S}^{\prime}\left(\mathbb{R}^{d} \rightarrow \mathbb{R}\right), d=2,3$, (obtained in Example 1). For $d=2$, let : $Z^{4}:$ be the $\mathcal{S}^{\prime}$ valued random variable on $\left(\mathcal{S}^{\prime}, \mathcal{B}\left(\mathcal{S}^{\prime}\right), v_{0}\right)$ uniquely defined by (see, e.g., [89], and for a definition by means of the multiple stochastic integrals, cf., e.g. [8,36]). also cf. Remark 7)

$$
\begin{aligned}
& \int_{\mathcal{S}^{\prime}} \mathcal{S}^{\prime}<: Z^{4}:, h>\mathcal{S}: \mathcal{S}^{\prime}<\phi, \varphi_{1}>\mathcal{S} \cdots \mathcal{S}^{\prime}<\phi, \varphi_{4}>\mathcal{S}: v_{0}(d \phi) \\
& \quad=\int_{\mathbb{R}^{2}} \prod_{j=1}^{4}\left(\int_{\mathbb{R}^{2}}\left(-\Delta+m_{0}^{2}\right)^{-1}\left(x-y_{j}\right) \varphi_{j}\left(y_{j}\right) d y_{j}\right) h(x) d x, \quad h, \varphi_{j} \in \mathcal{S}, j=1, \ldots, 4,
\end{aligned}
$$

where $h, \varphi_{j} \in \mathcal{S}, j=1, \cdots, 4$, the Wick power : $\mathcal{S}^{\prime}<\phi, \varphi_{1}>\mathcal{S} \cdots \mathcal{S}^{\prime}<\phi, \varphi_{4}>\mathcal{S}$ : is defined through the Hermite polynomials, e.g., a simple case : $\left(\mathcal{S}^{\prime}<\phi, \varphi>\mathcal{S}\right)^{4}:=$ $\sum_{n=0}^{2} \frac{4 !}{n !(4-2 n) !}\left(\mathcal{S}^{\prime}<\phi, \varphi>\mathcal{S}\right)^{4-2 n}\left(-\frac{a}{2}\right)^{n}$ with $a=\int_{\mathcal{S}^{\prime}}\left(\mathcal{S}^{\prime}<\phi, \varphi>\mathcal{S}\right)^{2} v_{0}(d \phi)$. For $d=3$, by putting a momentum cut-off $\kappa$ and an additional counter term (i.e., an additional mass renormalization, diverging when $\kappa$ is removed), similar to (5.30), an $\mathcal{S}^{\prime}\left(\mathbb{R}^{3} \rightarrow \mathbb{R}\right)$-valued random variable : $Z_{\kappa}^{4}$ : can be defined (cf. [42,43]).

In the case where $d=2$, for bounded regions $\Lambda \subset \mathbb{R}^{2}$, with $I_{\Lambda}$ the corresponding characteristic function, and a coupling constant $\lambda \geq 0$,

$$
v_{\Lambda}(d \phi) \equiv \frac{\exp \left(-\lambda<: Z^{4}:, I_{\Lambda}>\right)}{\int_{\mathcal{S}^{\prime}} \exp \left(-\lambda<: Z^{4}:, I_{\Lambda}>\right) v_{0}(d \phi)} v_{0}(d \phi),
$$

can be shown to be well defined (see $[52,74,89]$ ). Moreover, the existence of a weak limit $v^{*}$ (unique for small values of $\lambda$ ) such that

$$
v^{*} \equiv \lim _{\Lambda \uparrow \mathbb{R}^{2}} v_{\Lambda}
$$

has been proven (cf. e.g., [52,89], see also e.g., [29-31]). The probability measure $v^{*}$ on $\mathcal{S}^{\prime}\left(\mathbb{R}^{2} \rightarrow \mathbb{R}\right)$ defined by (5.32) is known as the $\Phi_{2}^{4}$ Euclidean field measure. 
Similarly, in case where $d=3$, by [43], the existence of a weak limit point $v^{*}$ (for adequately small $\lambda \geq 0$ ) such that

$$
v^{*} \equiv \lim _{\Lambda \uparrow \mathbb{R}^{3}} \lim _{\kappa \rightarrow 1} v_{\Lambda, \kappa}
$$

where

$$
v_{\Lambda, \kappa} \equiv \frac{\exp \left(-\lambda<: \tilde{Z}_{\kappa}^{4}:, I_{\Lambda}>\right)}{\int_{\mathcal{S}^{\prime}} \exp \left(-\lambda<: \tilde{Z}_{\kappa}^{4}:, I_{\Lambda}>\right) v_{0}(d \phi)} v_{0}(d \phi)
$$

with

$$
: \tilde{Z}_{\kappa}^{4}: \equiv: Z_{\kappa}^{4}:+\lambda a(\kappa)-\lambda^{3} b(\kappa), \text { for suitable constants } a, b \text { depending on } \kappa
$$

(cf. $[42,43]$ and $[22,23,54,54]$, also $(5.37)$ given below for a lattice formulation) has been proven. The probability measure $v^{*}$ on $\mathcal{S}^{\prime}\left(\mathbb{R}^{3} \rightarrow \mathbb{R}\right)$ defined by (5.33) is known as "the $\Phi_{3}^{4}$ Euclidean measure" (the uniqueness of limit points for sufficiently small value of $\lambda \geq 0$ is conjectured).

For $d=3$ the power 4 is a critical point for the existence of corresponding probability measures, and the analytic data of the $\Phi_{3}^{4}$ Euclidean field measure are quite singular. The Euclidean invariance is assured by the considerations in [43] (cf. also [Magnen,Sénéor 76], [52,86] and references therein). For $d=4$ there is no affirmative result on existence of $\Phi_{4}^{4}$ model, see however, e.g., [45,51], [Fröhlich], [Aiz1], [Aiz2], [GaR], [4,54,62].

The alternative procedure, through which the $\Phi_{d}^{4}$ Euclidean field measures are defined, is the following: Let $d=2,3$. For each bounded region $\Lambda \subset \mathbb{R}^{d}$ and a lattice spacing $\epsilon>0$, let

$$
L_{\epsilon, \Lambda} \equiv(\epsilon \mathbb{Z})^{d} \cap \Lambda,
$$

and define a family of real valued random variables $\phi \equiv\left\{\phi(x): x \in L_{\epsilon, \Lambda}\right\}$, the probability distribution of which is given by

$$
v_{\epsilon, \Lambda} \equiv \frac{1}{Z_{\epsilon, \Lambda}} \prod_{x \in L_{\epsilon, \Lambda}} e^{-S_{L_{\epsilon, \Lambda}}(\phi)} d \phi(x),
$$

where $Z_{\epsilon, \Lambda}$ is the normalizing constant,

$$
S_{L_{\epsilon, \Lambda}}(\phi) \equiv \frac{1}{2} \sum_{<x, y>} \epsilon^{d-2}(\phi(x)-\phi(y))^{2}+\frac{1}{2} a_{\epsilon} \sum_{x \in L_{\epsilon, \Lambda}} \epsilon^{d} \phi^{2}(x)+\frac{\lambda}{2} \sum_{x \in L_{\epsilon, \Lambda}} \epsilon^{d} \phi^{4}(x),
$$

with $a_{\epsilon}$ a counter term depending on $\epsilon>0$ and $d=2,3, \lambda \geq 0$ a coupling constant; $<x, y\rangle$ denotes the nearest neighbor points in $L_{\epsilon, \Lambda}$. In [38] it is shown, roughly speaking that, for adequately small $\lambda \geq 0$, there exists a subsequence $\left\{v_{\epsilon_{i}, \Lambda_{j}}\right\}_{i, j \in \mathbb{N}}$ of $\left\{v_{\epsilon, \Lambda}\right\}_{\epsilon>0, \Lambda \subset \mathbb{R}^{d}}$ with $\lim _{i \rightarrow \infty} \epsilon_{i}=0$ and $\lim _{j \rightarrow \infty} \Lambda_{j}=\mathbb{R}^{d}$, and a weak limit

$$
v^{*} \equiv \lim _{i \rightarrow \infty} \lim _{j \rightarrow \infty} v_{\epsilon_{i}, \Lambda_{j}}
$$

exists in the space of Borel probability measures on $\mathcal{S}^{\prime}\left(\mathbb{R}^{d} \rightarrow \mathbb{R}\right)$ by interpreting $v_{\epsilon, \Lambda}$ as an element in this space, for each $\epsilon>0$ and $\Lambda \subset \mathbb{R}^{d}$ (cf., the subsequent precise 
discussions from (5.39) to (5.56), for the weak convergence). For each $d=2,3, v^{*}$ given by (5.38) is a $\Phi_{d}^{4}, d=1,2,3$, Euclidean field measure, defined through a lattice approximation, see $[51,77,78,90]$.

The present example is formulated by using the $\Phi_{d}^{4}$ Euclidean field measure on $\mathcal{S}^{\prime}\left(\mathbb{R}^{d} \rightarrow \mathbb{R}\right)$ constructed through the lattice approximation described above. For this purpose, we need to certify the support properties of the measures with more details. Precisely, to apply Theorems 1, 2, 3 and 4 (cf. Example 1 and Theorem 5) to the example, we have to know that the supports of these measures are in some Hilbert spaces (see (5.48) below).

For each $\epsilon>0$, and bounded region $\Lambda \subset \mathbb{R}^{d}, d=1,2,3$, and for $F(\phi)$ a polynomial in $\left\{\phi(x): x \in L_{\epsilon, \Lambda}\right\}$, by (5.35) and (5.36) let

$$
<F>_{\epsilon, \Lambda} \equiv \int_{\mathbb{R}^{N(\epsilon, \Lambda)}} F(\phi) v_{\epsilon, \Lambda}(d \phi),
$$

where $N(\epsilon, \Lambda)$ is the cardinality of $L_{\epsilon, \Lambda}$. By [90] (cf. also section 2 of [38]), the following limit exists:

$$
<F>^{(\epsilon)} \equiv \lim _{\Lambda \uparrow \mathbb{R}^{d}}<F>_{\epsilon, \Lambda} .
$$

Also, for each $\epsilon>0$, and $d=1,2,3$, there exists a weak limit

$$
v_{\epsilon} \equiv \lim _{\Lambda \uparrow \mathbb{R}^{d}} v_{\epsilon, \Lambda},
$$

that is a Borel probability measure on $\mathcal{S}^{\prime}\left(\mathbb{R}^{d} \rightarrow \mathbb{R}\right)$. Let $D^{(\epsilon)}$ be the two point function of the lattice field with the lattice spacing $\epsilon>0$ and a given mass $m_{0}>0$, such that for $x, y \in(\epsilon \mathbb{Z})^{d}$

$$
D^{(\epsilon)}(x-y)=(2 \pi)^{-d} \int_{\left[-\frac{\pi}{\epsilon}, \frac{\pi}{\epsilon}\right]^{d}}\left(2 \epsilon^{-2} \sum_{i=1}^{d}\left(1-\cos \epsilon k_{i}\right)+m_{0}^{2}\right)^{-1} e^{i k \cdot(x-y)} d k_{1} \cdots d k_{d},
$$

which is the lattice version of the corresponding covariance operator $\left(-\Delta+m_{0}^{2}\right)^{-1}$ for the continuous Euclidean free field model (cf. (5.25), (5.26) and (5.30)), i.e., the so called free propagator (cf. Remark 7) which is denoted by $C^{\epsilon}(x-y)$ in [38]. By (5.40), define

$$
S^{(\epsilon)}(x-y) \equiv<\phi(x) \cdot \phi(y)>^{(\epsilon)}, \quad x, y \in(\epsilon \mathbb{Z})^{d},
$$

and

$$
S_{n}^{(\epsilon)}\left(x_{1}, \ldots, x_{n}\right) \equiv<\prod_{i=1}^{n} \phi\left(x_{i}\right)>^{(\epsilon)}, \quad x_{i} \in L_{\epsilon}, i=1, \ldots, n, n \in \mathbb{N} .
$$

From Theorem 6.1, Lemma A.1 with the formulas (A.13), (A.16), (A.17) and (8.2) in [38], where $m_{0}^{2}$ is taken to be equal 1 and by setting $\alpha=0$, we see that there exist universal constants $\lambda_{0}, K_{1}, K_{2}$ such that if $0 \leq \lambda \leq \lambda_{0}$, then we have the inequalities

$$
\left|\left\|S^{(\epsilon)}-D^{(\epsilon)}\right\|\right|^{(\epsilon)} \leq K_{1} \lambda^{2}, \quad \forall \epsilon>0
$$

and

$$
\|\left. D^{(\epsilon)}\right|_{l^{1}} ^{(\epsilon)} \leq K_{2} m_{0}^{-2}, \quad \forall \epsilon>0
$$


Moreover the Gaussian inequality

$$
\begin{gathered}
0 \leq S_{2 n}^{(\epsilon)}\left(x_{1}, \ldots, x_{2 n}\right) \leq \sum_{\text {pairing }} \prod S^{(\epsilon)}\left(x_{i}, x_{j}\right), \\
S_{2 n-1}^{(\epsilon)}\left(x_{1}, \ldots, x_{2 n-1}\right)=0, \quad n \in \mathbb{N}
\end{gathered}
$$

holds, where the indices $i$ and $j$ move in $\{1, \ldots, 2 n\}$ and the notation such that $\sum_{\text {pairing }} \prod$ is defined in Remark 7, and

$$
|\|f\||^{(\epsilon)} \equiv\|f\|_{l^{1}}^{(\epsilon)}+\|f\|_{\infty}^{(\epsilon)} \equiv \epsilon^{d} \sum_{x \in(\epsilon \mathbb{Z})^{d}}|f(x)|+\sup _{x \in(\epsilon \mathbb{Z})^{d}}|f(x)| .
$$

By Remark 7, we note that the inequality in (5.46) holds as the equality for the Euclidean free field model. By making use of (5.44), (5.45) and (5.46), for $d=1,2,3$, by applying Theorem 5 we shall prove below that the supports of the Borel probability measure $v_{\epsilon}$ on $\mathcal{S}^{\prime}\left(\mathbb{R}^{d} \rightarrow \mathbb{R}\right)$, for each $\epsilon>0$, and of a weak limit of a subsequence of $\left\{v_{\epsilon}\right\}_{\epsilon>0}$, denoted by $v$, are all in the Hilbert space $\mathcal{H}_{-2} \subset \mathcal{S}^{\prime}\left(\mathbb{R}^{d} \rightarrow \mathbb{R}\right)$ defined by (5.11) so that from now on

$$
v_{\epsilon} \text { and } v \text { can be understood as probability measures on } \mathcal{H}_{-2} \text {. }
$$

In fact, by the Sobolev's embedding theorem and by (5.9) with (5.7) and (5.10), we see that there exist some constants $K_{3}, K_{4}, K_{5}$ and the following inequality with respect to the norms, and the corresponding continuous embeddings hold:

$$
\begin{aligned}
& \sup _{x \in \mathbb{R}^{d}}\left|\left(|x|^{2}+1\right)^{\frac{d+1}{2}} f(x)\right| \leq K_{3}\left\|\left(|x|^{2}+1\right)^{\frac{d+1}{2}} f\right\|_{W^{d+1,2}} \\
& \leq K_{4}\left\|(-\Delta+1)^{\frac{d+1}{2}}\left(|x|^{2}+1\right)^{\frac{d+1}{2}} f\right\|_{L^{2}\left(\mathbb{R}^{2}\right)} \leq K_{5}\|f\|_{\mathcal{H}_{1}}, \quad \forall f \in \mathcal{S}\left(\mathbb{R}^{d} \rightarrow \mathbb{R}\right), \\
& \quad \mathcal{H}_{1} \hookrightarrow\left(|x|^{2}+1\right)^{-\frac{d+1}{2}} W^{d+1,2}\left(\mathbb{R}^{d}\right) \hookrightarrow\left(|x|^{2}+1\right)^{-\frac{d+1}{2}} C_{b}\left(\mathbb{R}^{d} \rightarrow \mathbb{R}\right),
\end{aligned}
$$

where $W^{d+1,2}=W^{d+1,2}\left(\mathbb{R}^{d}\right)$ is the Sobolev space, the elements of which are real measurable functions having square integrable (with respect to the Lebesgue measure on $\mathbb{R}^{d}$ ) partial derivatives (in the sense of distribution) of all orders up to $d+1$, and $C_{b}\left(\mathbb{R}^{d} \rightarrow \mathbb{R}\right)$ is the space of real valued continuous bounded functions on $\mathbb{R}^{d}$.

Remark 8. By refining the above discussion, passing through similar arguments, it is possible to get sharper results than (5.49) (cf. [10] for considerations on the path properties of corresponding Euclidean fields, cf., also references therein), but (5.49) is sufficient for the subsequent discussions, in particular, for (5.50) below.

Then, by Young's inequality, which is valid for both integrals and sums on lattices (cf., e.g., (A.3) in Appendix of [38]), from (5.44), (5.45), (5.46), (5.47) and (5.49), we see that (cf., (5.41) and (5.42), cf. also (5.26)) there exist constants $K^{\prime}, K$ such that

$$
\begin{aligned}
& \left|\int_{\mathcal{S}^{\prime}}\left(\mathcal{S}^{\prime}<\phi, \varphi>\mathcal{S}^{\prime}\right)^{2} v_{\epsilon}(d \phi)\right| \\
& \quad=\left|\epsilon^{d} \sum_{x \in(\epsilon \mathbb{Z})^{d}} \varphi(x)\left(\epsilon^{d} \sum_{y \in(\epsilon \mathbb{Z})^{d}} S^{(\epsilon)}(x-y) \varphi(y)\right)\right|
\end{aligned}
$$




$$
\begin{aligned}
& \leq\left|\epsilon^{d} \sum_{x \in(\epsilon \mathbb{Z})^{d}}\right| \varphi(x)\left|\left(\epsilon^{d} \sum_{y \in(\epsilon \mathbb{Z})^{d}} S^{(\epsilon)}(x-y)|\varphi(y)|\right)\right| \\
& \leq K^{\prime}\left(\epsilon^{d} \sum_{x \in(\epsilon \mathbb{Z})^{d}}|\varphi(x)|\right) \cdot\left(K_{1} \lambda^{2}+K_{2} m_{0}^{-2}\right) \cdot\|\varphi\|_{\infty}^{(\epsilon)} \\
& \leq K^{\prime} \cdot K_{5}^{2}\left(\epsilon^{d} \sum_{x \in(\epsilon \mathbb{Z})^{d}}\|\varphi\|_{\mathcal{H}_{1}}^{2}\left(|x|^{2}+1\right)^{-\frac{d+1}{2}}\right)\left(K_{1} \lambda^{2}+K_{2} m_{0}^{-2}\right) \\
& \leq K\|\varphi\|_{\mathcal{H}_{1}}^{2}, \quad \forall \epsilon>0, \forall \varphi \in \mathcal{H}_{1},
\end{aligned}
$$

where \|\|$_{\mathcal{H}_{1}}$ is the $\mathcal{H}_{1}$ norm defined by (5.11), and we have used the simplified notations $\mathcal{S}=\mathcal{S}\left(\mathbb{R}^{d} \rightarrow \mathbb{R}\right), \mathcal{S}^{\prime}=\mathcal{S}^{\prime}\left(\mathbb{R}^{d} \rightarrow \mathbb{R}\right)$. In (5.50) for the third inequality, we used Young's inequality to get

$$
\exists K^{\prime} \text { such that }\left\|S^{(\epsilon)} * \varphi\right\|_{\infty}^{(\epsilon)} \leq K^{\prime}\left\|S^{(\epsilon)}\right\|_{l^{1}}^{(\epsilon)} \cdot\|\varphi\|_{\infty}^{(\epsilon)}
$$

and for the last but one inequality, we used the following consequence from (5.49)

$$
\begin{aligned}
|\varphi(x)| \leq & \left(\sup _{x^{\prime} \in \mathbb{R}^{d}}\left(\left|x^{\prime}\right|^{2}+1\right)^{\frac{d+1}{2}}\left|\varphi\left(x^{\prime}\right)\right|\right) \cdot\left(|x|^{2}+1\right)^{-\frac{d+1}{2}} \leq K_{5}\|\varphi\|_{\mathcal{H}_{1}}\left(|x|^{2}+1\right)^{-\frac{d+1}{2}}, \\
& \forall x \in \mathbb{R}^{d}, \quad \forall \varphi \in \mathcal{H}_{1} .
\end{aligned}
$$

By (5.50), from the Gaussian inequality (5.46) we have

$$
\begin{aligned}
& \left|\int_{\mathcal{S}^{\prime}}\left(\mathcal{S}^{\prime}<\phi, \varphi>\mathcal{S}^{\prime}\right)^{2 n} v_{\epsilon}(d \phi)\right| \\
& \quad=\left|\left(\epsilon^{d}\right)^{2 n} \sum_{x_{1}, \ldots, x_{2 n} \in(\epsilon \mathbb{Z})^{d}} \varphi\left(x_{1}\right) \cdots \varphi\left(x_{2 n}\right) S_{2 n}^{(\epsilon)}\left(x_{1}, \ldots, x_{2 n}\right)\right| \\
& \quad \leq(2 n-1) ! ! K^{n}\|\varphi\|_{\mathcal{H}_{1}}^{2 n}, \quad \forall \epsilon>0, \forall \varphi \in \mathcal{H}_{1}, n \in \mathbb{N} .
\end{aligned}
$$

To derive the second inequality of (5.51), we used the Gaussian inequality and recalled the last formula given in Remark 7. Now, by (5.51) (cf. (5.41), (5.42), (5.43)), we have (5.52) below, where the first equality holds, since $\lim _{N \rightarrow \infty} \sum_{n=1}^{N} \frac{(-1)^{n}}{(2 n) !}\left(\mathcal{S}^{\prime}<\phi, \varphi>\mathcal{S}^{\prime}\right)^{2 n}=$ $e^{i} \mathcal{S}^{\prime<\phi, \varphi>\mathcal{S}^{\prime}}-1, v_{\epsilon}$ a.e. $\phi$, by (5.51) since $\sum_{n=1}^{\infty} \frac{1}{(2 n) !} \int_{\mathcal{S}^{\prime}}\left(\mathcal{S}^{\prime}<\phi, \varphi>\mathcal{S}^{\prime}\right)^{2 n} v_{\epsilon}(d \phi)<$ $\infty$, holds, by Fubini's Lemma, also the second equality holds; the last inequality in (5.52) follows again by applying (5.51):

$$
\begin{aligned}
& \left|\int_{\mathcal{S}^{\prime}} e^{i} \mathcal{S}^{\prime}<\phi, \varphi>\mathcal{S}^{\prime} v_{\epsilon}(d \phi)-1\right| \\
& =\left|\int_{\mathcal{S}^{\prime}} \sum_{n=1}^{\infty} \frac{(-1)^{n}}{(2 n) !}\left(\mathcal{S}^{\prime}<\phi, \varphi>\mathcal{S}^{\prime}\right)^{2 n} v_{\epsilon}(d \phi)\right| \\
& =\left|\sum_{n=1}^{\infty} \frac{(-1)^{n}}{(2 n) !} \int_{\mathcal{S}^{\prime}}\left(\mathcal{S}^{\prime}<\phi, \varphi>\mathcal{S}^{\prime}\right)^{2 n} v_{\epsilon}(d \phi)\right| \\
& \leq \sum_{n=1}^{\infty} \frac{(2 n-1) ! !}{(2 n) !} K^{n}\|\varphi\|_{\mathcal{H}_{1}}^{2 n}=e^{\frac{1}{2} K\|\varphi\|_{\mathcal{H}_{1}}^{2}-1, \quad \forall \epsilon>0, \forall \varphi \in \mathcal{H}_{1} .}
\end{aligned}
$$


Denote, for any $\epsilon>0, \varphi \in \mathcal{S}$

$$
C^{(\epsilon)}(\varphi) \equiv \int_{\mathcal{S}^{\prime}} e^{i \mathcal{S}^{\prime<\phi, \varphi>} \mathcal{S}^{\prime}} v_{\epsilon}(d \phi) .
$$

Since $v_{\epsilon}$ is a Borel probability measure on $\mathcal{S}^{\prime}, C^{(\epsilon)}$ satisfies conditions ii) and iii) for $C$ in Theorem 5, the Bochner-Minlos theorem. Then, by (5.52) and Remark 6, given after Theorem 5, the continuity of $C^{(\epsilon)}(\cdot)$ on $\mathcal{H}_{1}$ follows immediatly. Namely, by Remark 6 (precisely, the last formula in the proof of Remark 6), and (5.52) the following holds:

$$
\left|C^{(\epsilon)}(\varphi)-C^{(\epsilon)}(\psi)\right|^{2} \leq 2 \cdot\left|C^{(\epsilon)}(\varphi-\psi)-1\right| \leq 2\left(e^{\left.\frac{1}{2} K\|\varphi-\psi\|_{\mathcal{H}_{1}}^{2}-1\right), \quad \forall \varphi, \psi \in \mathcal{H}_{1} .}\right.
$$

We thus see that $C^{(\epsilon)}(\cdot)$ is continuous with respect to the $\mathcal{H}_{1}$ norm \|\|$_{\mathcal{H}_{1}}$ (by the absolute estimate given by (5.51) and (5.52), it is also possible to show the continuity of $C^{(\epsilon)}$ (see Remark 10, given after this Example)). Hence, from i) of Theorem 5 with its last statement (cf. the arguments between (5.14) and (5.18)), we conclude that the support of $v_{\epsilon}$ is in $\mathcal{H}_{-2}$ for any $\epsilon>0$. This guarantees (5.48) for $v_{\epsilon}$.

To certify (5.48) for a weak limit of $\left\{v_{\epsilon}\right\}_{\epsilon>0}$, as $\epsilon \downarrow 0$, obtained from (5.50), (5.51) and (5.46), we recall that the distribution $S_{k}^{(\epsilon)}, k \in \mathbb{N}, \epsilon>0$, satisfies

$$
S_{2 n-1}^{(\epsilon)}=0 \quad \text { and }\left\|S_{2 n}^{(\epsilon)}\right\|_{\left(\mathcal{H}_{-1}\right) \otimes 2 n} \leq(2 n-1) ! ! K^{n}, \quad \forall \epsilon>0, \quad \forall n \in \mathbb{N},
$$

Namely, for each $n \in \mathbb{N}$, the family of distributions $\left\{\mathcal{S}_{2 n}^{(\epsilon)}\right\}_{\epsilon>0}$ forms a bounded set in the Hilbert space $\left(\mathcal{H}_{-1}\right)^{\otimes 2 n}$, the space of $2 n$-th tensor powers of $\mathcal{H}_{-1}$ defined by (5.11). Thus, for each $n \in \mathbb{N}$, we can take a sequence $\left\{\epsilon_{n, i}\right\}_{i \in \mathbb{N}}$, with $\epsilon_{n, i}>0$ and $\lim _{i \rightarrow \infty} \epsilon_{n, i}=0$ such that $\left\{\mathcal{S}_{2 n}^{\left(\epsilon_{n, i}\right)}\right\}_{i \in \mathbb{N}}$ converges weakly (as $\left.i \rightarrow \infty\right)$ to some $S_{2 n} \in\left(\mathcal{H}_{-1}\right)^{\otimes 2 n}$, that satisfies the same bound as (5.51). By taking subsequences and using a diagonal argument, we then see that there exists a sequence $\left\{\epsilon_{i}\right\}_{i \in \mathbb{N}}$ with $\epsilon_{i}>0$ and $\lim _{i \rightarrow \infty} \epsilon_{i}=0$ such that $\left\{S_{2 n}^{\epsilon_{i}}\right\}_{i \in \mathbb{N}}$ converges weakly (as $\left.i \rightarrow \infty\right)$ to $S_{2 n} \in\left(\mathcal{H}_{-1}\right)^{\otimes 2 n}$ for any $n \in \mathbb{N}$. By these, we can define the functional

$$
C(\varphi) \equiv \sum_{n=0}^{\infty} \frac{(-1)^{n}}{(2 n) !}\left\langle S_{2 n}, \varphi^{\otimes 2 n}\right\rangle,
$$

that is an absolutely convergent series and satisfies the same bound as (5.52):

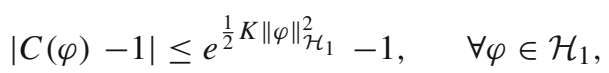

where $<S_{2 n}, \varphi^{\otimes 2 n}>={ }_{\left(\mathcal{H}_{-1}\right)^{\otimes 2 n}}\left\langle S_{2 n}, \varphi^{\otimes 2 n}\right\rangle_{\left(\mathcal{H}_{1}\right)^{\otimes 2 n}}$ is the dualization between $\left(\mathcal{H}_{-1}\right)^{\otimes 2 n}$ and $\left(\mathcal{H}_{1}\right)^{\otimes 2 n}$. For each $\epsilon>0$, since $C^{(\epsilon)}$ defined by (5.53) satisfies the conditions i), ii), iii) of Theorem 5, by the construction $C(\varphi)$ defined by (5.55) it also satisfies the same conditions. In particular, by $(5.56), C(\varphi)$ is continuous with respect to the $\mathcal{H}_{1}$ norm (precisely, see the explanation given after (5.53), also cf. Remark 10 given after this example). Hence, from Theorem 5, we deduce the existence of a Borel probability measure $v$ on $\mathcal{H}_{-2}$ corresponding to $C(\varphi)$ defined by (5.55). This guarantees (5.48) for $v$. From now on we fix $v$ to be the probability measure as follows:

$v$ is the probability measure on $\mathcal{H}_{-2}$ corresponding to $C(\varphi)$ defined by (5.55). 
We thus arrive at a situation analogous to the one in Example 1. On the space $L^{2}\left(\mathcal{H}_{-2}, v\right)$ with the probability measure $v$ defined by $(5.57)$, we can construct an $\mathcal{H}_{-3^{-}}$ valued Hunt process that is a stochastic quantization of the Euclidean $\Phi_{d}^{4}, d=2,3$, field with respect to a non-local Dirichlet form.

Precisely, we repeat the analogous discussions between (5.27) and (5.29) (cf. also the susequent discussions for the case where $\alpha \in(0,1)$ given after (5.29)). As was done in Example 1, we interpret $v$ defined by (5.57) as the Borel probability measure on $\mathcal{H}_{-3}$ (that is wider space than the original domain $\left.\mathcal{H}_{-2}\right)$. By (5.22), (5.23) and (5.24), by taking $m=-3, \tau_{-3}$ defines an isometric isomorphism such that

$$
\begin{gathered}
\tau_{-3}: \mathcal{H}_{-3} \ni f \longmapsto\left(\lambda_{1}^{-3} a_{1}, \lambda_{2}^{-3} a_{2}, \ldots\right) \in l_{\left(\lambda_{i}^{6}\right)}^{2}, \\
\text { with } \quad a_{i} \equiv\left(f, \lambda_{i}^{-3} \varphi_{i}\right)_{-3}, \quad i \in \mathbb{N} .
\end{gathered}
$$

Define a probability measure $\mu$ on $l_{\left(\lambda_{i}^{6}\right)}^{2}$ such that

$$
\mu(B) \equiv \nu \circ \tau_{-3}^{-1}(B) \text { for } B \in \mathcal{B}\left(l_{\left(\lambda_{i}^{6}\right)}^{2}\right) .
$$

We set $S=l_{\left(\lambda_{i}^{6}\right)}^{2}$ in Theorems 1, 2 and 4, then it follows that for the weight $\beta_{i}$ we have $\beta_{i}=\lambda_{i}^{6}$. We can take $\gamma_{i}^{-\frac{1}{2}}=\lambda_{i}$ in Theorem 2-i) with $p=2$, then, from (5.18) we get

$$
\begin{aligned}
& \sum_{i=1}^{\infty}\left(\beta_{i} \gamma_{i}\right)^{\frac{\alpha+1}{2}} \cdot \mu\left(\beta_{i}^{\frac{1}{2}}\left|X_{i}\right|>M \cdot \gamma_{i}^{-\frac{1}{2}}\right) \\
& \quad \leq \sum_{i=1}^{\infty}\left(\beta_{i} \gamma_{i}\right)^{\frac{\alpha+1}{2}}=\sum_{i=1}^{\infty}\left(\lambda_{i}\right)^{2(\alpha+1)}<\infty, \quad \forall \alpha \in(0,1] .
\end{aligned}
$$

(5.60) shows that the condition (4.3) holds.

Also, as has been mentioned above, since $v\left(\mathcal{H}_{-n}\right)=1$, for any $n \geq 2$, we have

$$
\begin{aligned}
& 1=\nu\left(\mathcal{H}_{-2}\right)=\mu\left(l_{\left(\lambda_{i}^{4}\right)}^{2}\right)=\mu\left(\bigcup_{M \in \mathbb{N}}\left\{\left|X_{i}\right| \leq M \beta_{i}^{-\frac{1}{2}} \gamma_{i}^{-\frac{1}{2}}, \forall i \in \mathbb{N}\right\}\right), \\
& \quad \text { for } \beta_{i}=\lambda_{i}^{6}, \gamma_{i}^{-\frac{1}{2}}=\lambda_{i} .
\end{aligned}
$$

This shows that the condition (4.4) is satisfied.

Thus, by Theorem 2-i) and Theorem 4, for each $0<\alpha \leq 1$, there exists an $l_{\left(\lambda_{i}^{6}\right)}^{2}$-valued Hunt process

$$
\mathbb{M} \equiv\left(\Omega, \mathcal{F},\left(X_{t}\right)_{t \geq 0},\left(P_{\mathbf{x}}\right)_{\mathbf{x} \in S_{\triangle}}\right),
$$

associated to the non-local Dirichlet form $\left(\mathcal{E}_{(\alpha)}, \mathcal{D}\left(\mathcal{E}_{(\alpha)}\right)\right)$. We can then define an $\mathcal{H}_{-3^{-}}$ valued process $\left(Y_{t}\right)_{t \geq 0}$ such that $\left(Y_{t}\right)_{t \geq 0} \equiv\left(\tau_{-2}^{-1}\left(X_{t}\right)\right)_{t \geq 0}$. Equivalently, by (5.58) for $X_{t}=\left(X_{1}(t), X_{2}(t), \ldots\right) \in l_{\left(\lambda_{i}^{6}\right)}^{2}, P_{\mathbf{x}}-$ a.e. $x \in S_{\triangle}$, by setting $A_{i}(t)$ such that $X_{i}(t)=$ $\lambda_{i}^{-3} A_{i}(t)$ (cf. (5.21) and (5.22)), then $Y_{t}$ is given by

$$
Y_{t}=\sum_{i \in \mathbb{N}} A_{i}(t)\left(\lambda_{i}^{-3} \varphi_{i}\right)=\sum_{i \in \mathbb{N}} X_{i}(t) \varphi_{i} \in \mathcal{H}_{-3}, \quad \forall t \geq 0, \quad P_{\mathbf{x}}-\text { a.e. }, x \in S_{\triangle}(5.62)
$$


By (5.4) and (5.58), $Y_{t}$ is an $\mathcal{H}_{-3}$-valued Hunt process that is a stochastic quantization with respect to the non-local Dirichlet form $\left(\tilde{\mathcal{E}}_{(\alpha)}, \mathcal{D}\left(\tilde{\mathcal{E}}_{(\alpha)}\right)\right)$ on $L^{2}\left(\mathcal{H}_{-3}, v\right)$, that is defined through $\left(\mathcal{E}_{(\alpha)}, \mathcal{D}\left(\mathcal{E}_{(\alpha)}\right)\right)$, by making use of $\tau_{-3}$ via (5.59). We state the above results as a theorem:

Theorem 6. Let $v$ be the Euclidean $\Phi_{d}^{4}, d=2,3$, field measure defined by (5.57). Interpret $v$ as a Borel probability measure on $\mathcal{H}_{-3}$, and let $\mu$ be the Borel probability measure on $l_{\left(\lambda_{i}^{6}\right)}^{2}$ that is an image of $v$ defined by (5.59). Then, for any $0<\alpha \leq 1$, on $L^{2}\left(l_{\left(\lambda_{i}^{6}\right)}^{2} ; \mu\right)$, a family of non-local quasi-regular Dirichlet form $\left(\mathcal{E}_{(\alpha)}, \mathcal{D}\left(\mathcal{E}_{(\alpha)}\right)\right)$ can be defined through Theorem 1 and Theorem 2-i), and by Theorem 4, there exists an $S \equiv l_{\left(\lambda_{i}^{6}\right)}^{2}$-valued Hunt process $\mathbb{M} \equiv\left(\Omega, \mathcal{F},\left(X_{t}\right)_{t \geq 0},\left(P_{\mathbf{x}}\right)_{\mathbf{x} \in S_{\triangle}}\right)$ properly associated to the Dirichlet form $\left(\mathcal{E}_{(\alpha)}, \mathcal{D}\left(\mathcal{E}_{(\alpha)}\right)\right)\left(c f\right.$. (5.61)). Moreover, the stochastic process $\left(Y_{t}\right)_{t \geq 0}$ defined by (5.62) through $\mathbb{M}$ is an $\mathcal{H}_{-3}$-valued Hunt process that is a stochastic quantization with respect to the non-local Dirichlet form $\left(\tilde{\mathcal{E}}_{(\alpha)}, \mathcal{D}\left(\tilde{\mathcal{E}}_{(\alpha)}\right)\right)$ on $L^{2}\left(\mathcal{H}_{-3}, v\right)$, that is defined through $\left(\mathcal{E}_{(\alpha)}, \mathcal{D}\left(\mathcal{E}_{(\alpha)}\right)\right)$ by making use of $\tau_{-3}$ defined by (5.58).

Remark 9. (5.52) is an analogue to (12.5.1) in [52] holding for (general) Euclidean $P(\phi)_{2}$ measures.

Remark 10. By (5.51), since $\sum_{n=0}^{\infty} \frac{(-1)^{n}}{(2 n) !} \int_{\mathcal{S}^{\prime}}\left(\mathcal{S}^{\prime}<\phi, \varphi>\mathcal{S}^{\prime}\right)^{2 n} v_{\epsilon}(d \phi)$, and $C(\varphi) \equiv$ $\sum_{n=0}^{\infty} \frac{(-1)^{n}}{(2 n) !}\left\langle S_{2 n}, \varphi^{\otimes 2 n}\right\rangle$, converge absolutely, for $\varphi, \psi \in \mathcal{S}$ we are admitted to perform the following evaluation (cf. (5.53)), and see, for e.g., the continuity of $C^{(\epsilon)}(\varphi)$ with respect to $\varphi \in \mathcal{H}_{1}$ :

$$
\begin{aligned}
& \left|C^{(\epsilon)}(\varphi)-C^{(\epsilon)}(\psi)\right|=\left|\int_{\mathcal{S}^{\prime}} e^{i \mathcal{S}^{\prime}<\phi, \varphi>\mathcal{S}^{\prime}} v_{\epsilon}(d \phi)-\int_{\mathcal{S}^{\prime}} e^{i \mathcal{S}^{\prime<\phi, \psi>} \mathcal{S}^{\prime}} v_{\epsilon}(d \phi)\right| \\
& =\left|\int_{\mathcal{S}^{\prime}} e^{i \mathcal{S}^{\prime}<\phi, \varphi>\mathcal{S}^{\prime}}\left(1-e^{i \mathcal{S}^{\prime}<\phi, \psi-\varphi>\mathcal{S}^{\prime}}\right) v_{\epsilon}(d \phi)\right| \\
& \leq\left|\int_{\mathcal{S}^{\prime}} \sum_{n=1}^{\infty} \frac{(-1)^{n}}{(2 n) !}\left(\mathcal{S}^{\prime}<\phi, \psi-\varphi>\mathcal{S}^{\prime}\right)^{2 n} v_{\epsilon}(d \phi)\right| \\
& \leq \sum_{n=1}^{\infty} \frac{(2 n-1) ! !}{(2 n) !} K^{n}\|\varphi-\psi\|_{\mathcal{H}_{1}}^{2 n}=e^{\frac{1}{2} K\|\varphi-\psi\|_{\mathcal{H}_{1}}^{2}-1, \quad \forall \epsilon>0 .}
\end{aligned}
$$

In the above, for the inequality of the third line we make use of the Hölder inequality with $\left|e^{i} \mathcal{S}^{\prime<\phi, \varphi>} \mathcal{S}^{\prime}\right|=1$, and the evaluation of the fourth line holds by (5.51), which follows from the Gaussian inequality (see (5.46)).

We note that for the Poisson random fields the Gaussian inequality does not hold (see [A,Gottschalk,Wu-j.L. 97] for the corresponding detailed discussions).

A martingale representation of Markov processes properly associated to Dirichlet forms is known as Fukushima decomposition (for the case where the state spaces $S$ are locally compact metric spaces cf., e.g., [46,48], and Theorem 4.3 of [49], and for the case where $S$ are general Hausdorff topological spaces cf., e.g., Chapter VI of [MR 92] and [32]). Corresponding to Fukushima decomposition, for Theorem 4 and Examples 1, 2 we have the following Remarks 11 and 12.

Remark 11 (Fukushima decomposition). The subspace of $S$, in which the $\mu$-tight special standard process and Hunt process properly associated with the quasi-regular Dirichlet 
form and strictly quasi-regular Dirichlet form on $L^{2}(S ; \mu)$ takes values, respectively, can be embedded in some locally compact separable metric space (cf. Definition IV-3.1 and Theorem VI-1.2 of [M,R 93], and also cf. the proof of Theorem 2 of the present paper). In what follows, the interpretation of Theorem 5.2.2 of [48] is straightforward, and then Theorem VI-2.5 of [M,R 93] holds.

We use the notions and notations adopted in chapter VI of [M,R 93] (cf. also the notations in chapter 5 of [48] and [25-27,32] and references therein).

Let $\mathbb{M} \equiv\left(\Omega, \mathcal{F},\left(X_{t}\right)_{t \geq 0},\left(P_{\mathbf{x}}\right)_{\mathbf{x} \in S_{\triangle}}\right)$ be the Hunt process defined through Theorem 4 . By a direct application of Theorem VI-2.5 of [M,R 93] we see that for $u \in \mathcal{D}\left(\mathcal{E}_{(\alpha)}\right)$, there exists a unique martingale additive functional of finite energy (MAF) $M^{[u]}$ and a continuous additive functional of zero energy (CAF's zero energy) $N^{[u]}$ such that

$$
A^{[u]}=M^{[u]}+N^{[u]},
$$

where

$$
A^{[u]} \equiv\left(A_{t}^{[u]}\right)_{t \geq 0}, \quad A_{t}^{[u]}=\tilde{u}\left(X_{t}\right)-\tilde{u}\left(X_{0}\right),
$$

with $\tilde{u}$ an $\mathcal{E}_{(\alpha)}$-quasi continuous $\mu$-version of $u \in \mathcal{D}\left(\mathcal{E}_{(\alpha)}\right)$. The decomposition formula (5.63) holds for Examples 1 and 2.

In order to consider the martingale problems (cf., e.g., [49]) corresponding to the decomposition given by (5.63), some additional assumptions for the probability measure $\mu$, e.g., a uniform regularity of its density function (cf. (2.11)), are necessary. Considerations in this direction can be found in [19].

Remark 12 (Subordination correspondences). A theory of transforming Dirichlet forms and associated symmetric Markov processes by means of the subordinations has been developed both from a functional analytic and a probabilistic point of view (for the case where the state spaces are locally compact spaces, cf. [64,83] and references therein, and for the case where the state space are general Hausdorff spaces, cf. [32]).

On $L^{2}(S ; \mu)$ with $\mu$ a Borel probability measure on a general Hausdorff topological space $S$, consider in general a quasi-regular Dirichlet form $(\mathcal{E}, \mathcal{D}(\mathcal{E}))$. Let $-L$ be the self-adjoint operator corresponding to $\left(\mathcal{E}, \mathcal{D}(\mathcal{E})\right.$ ) (thus, $\operatorname{Dom}(\sqrt{-L})=\mathcal{D}(\mathcal{E})$ ), and $P_{\lambda}$ be the projection valued measure associated to the operator $-L$. Let $f$ be a Bernstein function on $\mathbb{R}_{+}$(cf., e.g., [83] for its definition and properties), and define a self-adjoint operator $L^{f}$ by

$$
L^{f} \equiv-f(-L)=-\int_{0}^{\infty} f(\lambda) d P_{\lambda} .
$$

In [32] the following mathematical structure of the correspondences between subordinate symmetric processes and the subordinate sub-Markov semi-groups, and hence the associated Dirichlet forms, is introduced (see Theorems 2.7, 2.9, equations (8)(8), (9), Theorem 2.16 and Theorem 3.1 of [A,Rüdiger]): For a quasi-regular Dirichlet form $(\mathcal{E}, \mathcal{D}(\mathcal{E}))$ as above

$$
\mathcal{E}^{f}(\phi, \psi) \equiv(\sqrt{f(-L)} \phi, \sqrt{f(-L)} \psi)_{L^{2}(S ; \mu)}, \phi, \psi \in \mathcal{D}\left(\mathcal{E}^{f}\right) \equiv \operatorname{Dom}(\sqrt{f(-L)}),
$$

defines a non-local quasi-regular Dirichlet form. Let $\left(X_{t}^{f}\right)_{t \geq 0}$ be the $\mu$-tight special standard process properly associated to the quasi-regular Dirichlet form $\left(\mathcal{E}^{f}, \mathcal{D}\left(\mathcal{E}^{f}\right)\right)$, 
the corresponding semigroup of which is denoted by $\left(T_{t}^{f}\right)_{t \geq 0}$, then $\left(X_{t}^{f}\right)_{t \geq 0}$ has the same finite dimensional distributions as $\left(X_{y(t)}\right)_{t \geq 0}$, where $\left(X_{t}\right)_{t \geq 0}$ is the $\mu$-tight special standard process properly associated to the quasi-regular Dirichlet form $(\mathcal{E}, \mathcal{D}(\mathcal{E}))$ and $(y(t))_{t \geq 0}$ is the increasing Lévy process defined through the Bernstein function $f$.

By Theorem VI-2.5 of [M,R 93] (cf. Remark 11), the $\mu$-tight special standard process $\left(X_{t}^{f}\right)_{t \geq 0}$ admits the Fukushima decomposition (5.63). [32] discusses the Fukushima decomposition and the corresponding martingale problem for the process $\left(X_{t}^{f}\right)_{t \geq 0}$ (see Examples, 1, 2, 3, 4 and Theorem 4.29).

The investigations of the correspondences between the framework of the subordination and the general framework presented in the present work are particularly interesting and deserve further consideration.

\section{Future Developments}

Let us add some short comments on future developments corresponding to the present work, possibly encouraging future works by researchers working in related areas.

The present paper is intended to provide an explicit formulation of non-local Dirichlet forms defined on infinite dimensional topological vector spaces. Our definitions (2.8), (2.9) and (2.10) of the Markov symmetric form $\mathcal{E}_{(\alpha)}, 0<\alpha<2$, can be extended in several directions. In analogy with the finite dimensional cases (cf., e.g., [34,46,48, $49,60,70,84,88]$ and references therein), the kernel $\frac{1}{\left|y_{i}-y_{i}^{\prime}\right|^{\alpha+1}}$ can be replaced by more general symmetric ones (and also some non-symmetric ones).

Also the connections between the Hunt processes constructed by the non-local Dirichlet forms on infinite dimensional topological vector spaces and solutions of SPDEs seem to provide an area of possible extensions of our work (cf. e.g., [7] and references therein).

The study of contractivity properties of semi-groups with generators associates to Dirichlet forms is a particularly important subject. E.g., it is well known that the classical (local) Dirichlet forms, associated to certain Gaussian random fields, satisfy the logarithmic Sobolev inequality, which guarantees a spectral gap of the associated self adjoint operator and the semi-group corresponding to the operator satisfies the hypercontractivity (for corresponding precise results, cf. for [53,89], section X.9 of [1,80] and references therein). Analogous considerations for the non-local Dirichlet forms associated with random fields or processes on infinite dimensional topological vector spaces is another possible direction for future investigations.

\section{Dedication}

Dedicated to Professor Masatoshi Fukushima for his 88th birth day. Dedicated to Professor Rahael Høegh-Krohn for his 84th birth anniversary.

Acknowledgements. The authors would like to gratefully acknowledge the great hospitality of various institutions. In particular for the first named and second named authors, Giulio Casati and the Lake Como school of advanced studies, Complexity and Emergence: Ideas, Methods, with a special attention to Economics and Finance, Italy; for the third and fourth named authors, IAM and HCM at the University of Bonn, Germany; for the fourth named author, SFB 1283 and Bielefeld University, Germany. Also, the fourth named author expresses his strong acknowledgements to Professor Michael Röckner for fruitful discussions.

Open Access This article is licensed under a Creative Commons Attribution 4.0 International License, which permits use, sharing, adaptation, distribution and reproduction in any medium or format, as long as you give appropriate credit to the original author(s) and the source, provide a link to the Creative Commons licence, and indicate if changes were made. The images or other third party material in this article are included in the article's Creative Commons licence, unless indicated otherwise in a credit line to the material. If material is 
not included in the article's Creative Commons licence and your intended use is not permitted by statutory regulation or exceeds the permitted use, you will need to obtain permission directly from the copyright holder. To view a copy of this licence, visit http://creativecommons.org/licenses/by/4.0/.

Publisher's Note Springer Nature remains neutral with regard to jurisdictional claims in published maps and institutional affiliations.

\section{References}

[1] Aida, S.: Tunneling for spacially cut-off $P(\phi)_{2}$-Hamiltonians. J. Funct. Anal. 263, 2689-2753 (2012)

[2] Aizenman, M., Duminil-Copin, H., Marginal triviality of the scaling limits of critical 4D Ising and $\phi_{4}^{4}$ models. arXiv:1912.07973v3 [math-ph] 12 Mar 2021

[3] Albeverio, S., Theory of Dirichlet forms and applications. Lectures on probability theory and statistics (Saint-Flour, 2000), Lecture Notes in Math. 1816, 1-106, Springer, Berlin (2003)

[4] Albeverio, S., Along paths inspired by Ludwig Streit: stochastic equations for quantum fields and related systems. Stochastic and infinite dimensional analysis, 1-17, Trends Math., Birkhäuser/Springer, [Cham] (2016)

[5] Albeverio, S., De Vecchi, F., Gubinelli, M.: Elliptic stochastic quantization. Ann. Probab. 48, 1693-1741 (2020)

[6] Albeverio, S., De Vecchi, F., Gubinelli, M., On the elliptic stochastic quantization of some two dimensional Euclidean QFT's. To appare in Ann. Inst. H. Poincaré

[7] Albeverio, S., Di Persio, L., Mastrogiacomo, E., Smii, B.: A class of Lévy driven SDEs and their explicit invariant measures. Potential Anal. 45, 229-259 (2016)

[8] Albeverio, S., Ferrario, B., Yoshida, M.W.: On the essential self-adjointness of Wick powers of relativistic fields and of fields unitary equivalent to random fields. Acta Appl. Math. 80, 309-334 (2004)

[9] Albeverio, S., Figari, R.: Quantum fields and point interactions. Rend. Mat. Appl. 39, 161-180 (2018)

[10] Albeverio, S., Gielerak, R., Russo, F.: On the paths Hölder continuity in models of Euclidean quantum field theory. Stoch. Anal. Appl. 19, 677-702 (2001)

[11] Albeverio, S., Gottschalk, H., Wu, J.L.: Models of local relativistic quantum fields with indefinite metric (in all dimensions). Commun. Math. Phys. 184, 509-531 (1997)

[12] Albeverio, S., Hida, T., Potthoff, J., Streit, R., Röckner, M.: Dirichlet forms in terms of white noise analysis. I. Construction and QFT examples. Rev. Math. Phys. 1, 291-312 (1989)

[13] Albeverio, S., Hida, T., Potthoff, J., Streit, R., Röckner, M.: Dirichlet forms in terms of white noise analysis. II. Closability and diffusion processes. Rev. Math. Phys. 1, 313-323 (1989)

[14] Albeverio, S., Høegh-Krohn, R., Quasi invariant measures, symmetric diffusion processes and quantum fields. Les méthodes mathématiques de la théorie quantique des champs. (Colloq. Internat. CNRS, No. 248, Marseille, 1975) Éditions Centre Nat. Recherche Sci., Paris, 11-59 (1976)

[15] Albeverio, S., Høegh-Krohn, R.: Dirichlet forms and diffusion processes on rigged Hilbert spaces. Z. Wahrscheinlichkeitstheor. Verv. Geb. 40, 1-57 (1977)

[16] Albeverio, S., Høegh-Krohn, R.: Hunt processes and analytic potential theory on rigged Hilbert spaces. Ann. Inst. H. Poincaré Sect. B (N.S.) 13, 269-291 (1977)

[17] Albeverio, S., Høegh-Krohn, R., Streit, L.: Energy forms, Hamiltonians, and distorted Brownian paths. J. Math. Phys. 18, 907-917 (1977)

[18] Albeverio, S., Høegh-Krohn, R., Zegarlinski, B.: Uniqueness and global Markov property for Euclidean fields: the case of general polynomial interactions. Commun. Math. Phys. 123, 377-424 (1989)

[19] Albeverio, S., Kagawa, T., Kawasaki, S., Yahagi, Y., Yoshida, M.W., Non-local Markovian symmetric forms on infinite dimensional spaces, part 2, Part 2. Examples: non local stochastic quantization of space cut-off quantum fields and infinite particle systems. arXiv:2105.05593 (2021)

[20] Albeverio, S., Kawabi, H., Mihalache, S. R., Röckner, M., Strong uniqueness for Dirichlet operators related to stochastic quantization under exponential/trigonometric interactions on the two-dimensional torus. arXiv:2004.12383 [math.PR] (2021)

[21] Albeverio, S., Kondratiev, Y.G., Röckner, M.: Analysis and geometry on configuration spaces: the Gibbsian case. J. Funct. Anal. 157, 242-291 (1998)

[22] Albeverio, S., Kusuoka, S.: The invariant measures and the flow associated to the $\phi_{3}^{4}$-quantum field model. Ann SNPS Pisa 20, 1359-1427 (2020)

[23] Albeverio, S., Kusuoka, S.: Construction of a non-Gaussian and rotation-invariant $\phi^{4}$-measure and associated flow on $\mathbb{R}^{3}$ through stochastic quantization. arXiv:2102.08040 [math.PR], [math-ph], [math.AP] (2021)

[24] Albeverio, S., Liang, S., Zegarlinski, B.: Remark on the integration by parts formula for the $\phi_{3}^{4}$-quantum field model. Infinite Dim. Anal. Quantum Probab. Related Top. 9, 149-154 (2006) 
[25] Albeverio, S., Ma, Z.M., Röckner, M., Quasi regular Dirichlet forms and the stochastic quantization problem. Festschrift Masatoshi Fukushima, Interdiscip. Math. Sci., 17 (2015), 27-58, World Sci. Publ., Hackensack, NJ

[26] Albeverio, S., Ma, Z.M., Röckner, M.: Quasi-regular Dirichlet forms and Markov processes. J. Funct. Anal. 111, 118-154 (1993)

[27] Albeverio, S., Ma, Z.M., Röckner, M.: Local property of Dirichlet forms and diffusions on general state spaces. Math. Ann. 296, 677-686 (1993)

[28] Albeverio, S., Morato, L.M., Ugolini, S.: Non-symmetric diffusions and related Hamiltonians. Potential Anal. 8, 195-204 (1998)

[29] Albeverio, S., Röckner, M.: Classical Dirichlet forms on topological vector spaces- the construction of the associated diffusion processes. Probab. Theory Related Fields 83, 405-434 (1989)

[30] Albeverio, S., Röckner, M.: Classical Dirichlet forms on topological vector spaces-closability and a Cameron-Martin formula. J. Funct. Anal. 88, 43-395 (1990)

[31] Albeverio, S., Röckner, M.: Stochastic differential equations in infinite dimensions: solution via Dirichlet forms. Probab. Theory Related Fields 89, 347-386 (1991)

[32] Albeverio, S., Rüdiger, B.: Infinite-dimensional stochastic differential equations obtained by subordination and related Dirichlet forms. J. Funct. Anal. 204, 122-156 (2003)

[33] Albeverio, S., Rüdiger, B., Wu, J.-L., Analytic and probabilistic aspects of Lévy processes and fields in quantum theory. Lévy Processes, 187-224, Birkhäuser Boston, Boston, MA (2001)

[34] Albeverio, S., Song, S.: Closability and resolvent of Dirichlet forms perturbed by jumps. Potential Anal. 2(2), 115-130 (1993)

[35] Albeverio, S., Ugolini, S.: A Doob h-transform of the Gross-Pitaevskii Hamiltonian. J. Stat. Phys. 161, 486-508 (2015)

[36] Albeverio, S., Yoshida, M.W.: $H-C^{1}$ maps and elliptic SPDEs with polynomial and exponential perturbations of Nelson's Euclidean free field. J. Funct. Anal. 196, 265-322 (2002)

[37] Borkar, V.S., Chari, R.T., Mitter, S.K.: Stochastic quantization of field theory in finite and infinite volume. J. Funct. Anal. 81, 184-206 (1988)

[38] Brydges, D., Fröhlich, J., Sokal, A.: A New proof of the existence and non triviality of the continuum $\varphi_{2}^{4}$ and $\varphi_{3}^{4}$ quantum field theories. Commun. Math. Phys. 91, 141-186 (1983)

[39] Catellier, R., Chouk, K.: Paracontrolled distributions and the 3-dimensional stochastic quantization equation. Ann. Probab. 46, 2621-2679 (2018)

[40] Da Prato, G., Debussche, A.: Strong solutions to the stochastic quantization equations. Ann. Probab. 31, 1900-1916 (2003)

[41] Da Prato, G., Tubaro, L.: Self-adjointness of some infinite-dimensional elliptic operators and application to stochastic quantization. Probab. Theory Related Fields 118, 131-145 (2000)

[42] Feldman, J.: The $\lambda \varphi_{3}^{4}$ field theory in a finite volume. Commun. Math. Phys. 37, 93-120 (1974)

[43] Feldman, J., Osterwalder, K.: The Wightman axioms and the mass gap for weakly coupled $\left(\Phi^{4}\right)_{3}$ quantum field theories. Ann. Phys. 97, 80-135 (1976)

[44] Fröhlich, J.: On the triviality of $\lambda \phi_{d}^{4}$ theories and the approach to the critical point in $d_{(-)}>4$ dimensions. Nuclear Phys. B 200, 281-296 (1982)

[45] Fröhlich, J., Park, Y.M.: Remarks on exponential interactions and the quantum sine-Gordonequation in two space-time dimensions. Helv. Phys. Acta 50, 315-329 (1977)

[46] Fukushima, M.: Dirichlet Forms and Markov Processes, North-Holland Mathematical Library, 23. NorthHolland Publishing Co., Amsterdam (1980)

[47] Fukushima, M., Oshima, Y.: Recurrent Dirichlet forms and Markov property of associated Gaussian fields. Potential Anal. 49, 609-633 (2018)

[48] Fukushima, M., Oshima, Y., Takeda, M.: Dirichlet Forms and Symmetric Markov Processes, second revised and extended edn. de Gruyter, Berlin (2011)

[49] Fukushima, M., Uemura, T.: Jump-type Hunt processes generated by lower bounded semi- Dirichlet forms. Ann. Probab. 40, 858-889 (2012)

[50] Gallavotti, G., Rivasseau, V.: $\Phi^{4}$ field theory in dimension 4: a modern introduction to its unsolved problems. Ann. Inst. H. Poincaré Phys. Théor. 40, 185-220 (1984)

[51] Glimm, J., Jaffe, A.: Remark on existence of $\varphi_{4}^{4}$. Phys. Rev. Lett. 33, 440-442 (1974)

[52] Glimm, J., Jaffe, A.: Quantum Physics: A Functional Integral Point of View, 2nd edn. Springer, Berlin (1987)

[53] Gross, L., Logarithmic Sobolev inequalities and contractive properties of semigroups. Reprinted in Lecture Notes in Math. 1563, Springer, Berlin (1993)

[54] Gubinelli, M., Hofmanová, M.: Global solutions to elliptic and parabolic $\phi^{4}$ models in Euclidean space. Commun. Math. Phys. 368, 1201-1266 (2019)

[55] Gubinelli, M., Hofmanovà, M.: A PDE construction of the Euclidean $\Phi_{3}^{4}$ quantum field theory. Commun. Math. Phys. 384, 1-75 (2021) 
[56] Hairer, M.: A theory of regularity structures. Invent. Math. 198, 269-504 (2014)

[57] Hairer, M., Mattingly, J., The strong Feller property for singular stochastic PDEs. arXiv:1610.03415v1 (2016)

[58] Hida, T.: Brownian Motion. Springer, New York (1980)

[59] Hida, T., Kuo, H.-H., Potthoff, J., Streit, L.: White Noise. An Infinite-Dimensional Calculus. Mathematics and its Applications, vol. 253. Kluwer Academic Publishers Group, Dordrecht (1993)

[60] Hoh, W., Jacob, N.: On the Dirichlet problem for pseudodifferential operators generating feller semigroups. J. Funct. Anal. 137, 19-48 (1996)

[61] Itô, K.: Probability Theory, Iwanami kiso-suugaku sen-nshyo (in Japanese). Iwanami-Shyoten, Tokyo (1976)

[62] Itô, K.R.: Construction of four-dimensional quantum field models: $\phi_{4}^{4}$ and QED 4. Quantum Electrodyn., 15-35, Adv. Ser. Dir. High Energy Phys., 7, World Sci. Publ. (1989)

[63] Iwata, K.: Reversible measures of a $P(\phi)_{1}$-time evolution. Probabilistic methods in mathematical physics (Katata/Kyoto, 1985), Academic Press, Boston, MA (1987)

[64] Jacob, N., Schilling, R.: Function spaces as Dirichlet spaces (about a paper by Maz'ya and Nagel). J. Anal. Its Appl. 24, 3-28 (2005)

[65] Jona-Lasinio, G., Mitter, P.K.: On the stochastic quantization of field theory. Commun. Math. Phys. 101, 409-436 (1985)

[66] Jona-Lasinio, G., Mitter, P.K.: Large deviation estimates in the stochastic quantization of $\phi_{2}^{4}$. Commun. Math. Phys. 130, 111-121 (1993)

[67] Kolesnikov, A.V.: Mosco convergence of Dirichlet forms in infinite dimensions with changing reference measures. J. Funct. Anal. 230, 382-418 (2006)

[68] Kusuoka, S.: Dirichlet forms and diffusion processes on Banach space. J. Fac. Sci., Univ. Tokyo, Sect. IA. 29, 79-95 (1982)

[69] Ma, Z.M., Röckner, M.: Introduction to the Theory of (Non-Symmetric) Dirichlet Forms. Springer, Berlin (1992)

[70] Masamune, J., Uemura, T., Wang, J.: On the conservativeness and the recurrence of symmetric jumpdiffusions. J. Funct. Anal. 263, 3984-4008 (2012)

[71] Magnen, J., Sénéor, R.: The infinite volume limit of the $\phi_{3}^{4}$ model. Ann. Inst. H. Poincare Sect. A (N.S.) 24 95-159 (1976)

[72] Mizohata, S.: The Theory of Partial Differential Equations. Cambridge University Press, New York (1973)

[73] Mourrat, J.-C., Weber, H.: The Dynamic $\phi_{3}^{4}$ Model Comes Down from Infinity Commun. Math. Phys. 356, 673-753 (2017)

[74] Nelson, E.: A Quartic Interaction in Two Dimensions. In: Goodman, R., Segal, I. (eds) Mathematical Theory of Elementary Particles, M.I.T. Press, Cambridge, MA, pp. 69-73 (1966)

[75] Osada, H.: Dirichlet form approach to infinite-dimensional Wiener processes with singular interactions. Commun. Math. Phys. 176, 117-131 (1996)

[76] Parisi, G., Wu, Y.S.: Perturbation theory without gauge fixing. Sci. Sinica 24, 483-496 (1981)

[77] Park, Y.M.: The $\lambda \phi_{3}^{4}$ Euclidean quantum field theory in a periodic box. J. Math. Phys. 16, 2183-2188 (1975)

[78] Park, Y.M.: Convergence of lattice approximations and infinite volume limit in the $\left(\lambda \phi^{4}-\sigma \phi^{2}-\tau \psi\right)_{3}$ field theory. J. Math. Phys. 18, 354-366 (1977)

[79] Reed, M., Simon, B.: Methods of Modern Mathematical Physics. I. Functional Analysis. Academic Press, Cambridge (1978)

[80] Reed, M., Simon, B.: Methods of Modern Mathematical Physics. II. Fourier Analysis, self-Adjointness, Academic Press, Cambridge (1975)

[81] Röckner, M.: Generalized Markov fields and Dirichlet forms. Acta Appl. Math. 3, 285-311 (1985)

[82] Röckner, M., Zhu, R., Zhu, X.: Restricted Markov uniqueness for the stochastic quantization of $P(\Phi)_{2}$ and its applications. J. Funct. Anal. 125, 358-397 (2015)

[83] Schilling, R.L.: Subordination in the sense of Bochner and a related functional calculus. J. Austrial Math. Soc. Ser. A 64, 368-396 (1998)

[84] Schilling, R.L., Wang, J.: Lower Bounded Semi-Dirichlet Forms Associated with Levy Type Operators, Chapter 25. World Scientific, Festschritf Masatoshi Fukushima (2014)

[85] Schumuland, B.: An alternative compactification for classical Dirichlet forms on topological vector spaces. Stochast. Stochast. Rep. 33, 75-90 (1990)

[86] Seiler, E., Simon, B.: Nelson's symmetry and all that in the Yukawa2 and $\left(\phi^{4}\right)_{3}$ field theories. Ann. Phys. 97, 470-518 (1976)

[87] Shigekawa, I.: Semigroup domination on a Riemannian manifold with boundary. Recent developments in infinite-dimensional analysis and quantum probability. Acta Appl. Math. 63, 385-410 (2000) 
[88] Shiozawa, Y., Uemura, T.: Explosion of jump-type symmetric Dirichlet forms on $R^{d}$. J. Theoret. Probab. 27, 404-432 (2014)

[89] Simon, B.: The $P(\Phi)_{2}$ Euclidean (Quantum) Field Theory. Princeton University Press, Princeton, NJ (1974)

[90] Sokal, A.D.: An alternative constructive approach to $\phi_{3}^{4}$-quantum field theory, and a possible destructive approach to $\phi_{4}^{4}$. Ann. Inst. Henri Poincaré, A 37, 317-398 (1982)

[91] Trèves, F.: Topological Vector Spaces, Distributions and Kernels. Academic Press, New York (1967)

[92] Yoshida, M.W.: Construction of infinite-dimensional interacting diffusion processes through Dirichlet forms. Probab. Theory Relat. Fields 106, 265-297 (1996)

[93] Zegarlinski, B.: Uniqueness and global Markov property for Euclidean Fields: The case of general exponential interaction. Commun. Math. Phys. 96, 195-221 (1984)

[94] Zhu, R., Zhu, X.: Lattice approximation to the dynamical $\Phi_{3}^{4}$ model. Ann. Probab. 46, 397-455 (2018)

[95] Zhu, R., Zhu, X.: Dirichlet form associated with the $\Phi_{3}^{4}$ model. Electron. J. Probab. 23, 1-31 (2018)

Communicated by M. Hairer 